\title{
Advanced Parametric Modeling and Yield \\ Optimization Approaches for Microwave Passive \\ Components
}

\author{
by \\ Jianan Zhang, B.Eng \\ A thesis submitted to \\ the Faculty of Graduate and Postdoctoral Affairs \\ in partial fulfillment of the requirements for the degree of \\ Doctor of Philosophy
}

Electronic Science and Technology, School of Microelectronics Tianjin University, Tianjin, China

Ottawa-Carleton Institute for Electrical and Computer Engineering Carleton University, Ottawa, Ontario, Canada

(C) 2020

Jianan Zhang 


\begin{abstract}
Parametric modeling and yield optimization techniques play important roles in electromagnetic (EM)-based microwave component design. In the first part of this thesis, we propose a novel training approach for parametric modeling of microwave passive components with respect to changes in geometrical parameters using matrix Padé via Lanczos (MPVL) and EM sensitivities. In the proposed approach, the EM responses of passive components versus frequency are represented by polezero-gain transfer functions. The relationships between the poles/zeros/gain in the transfer function and geometrical variables are learnt by neural networks. A novel sensitivity-analysis-based pole/zero-matching algorithm is proposed to obtain the correct correspondences between the poles/zeros at different geometrical parameter values. Using the matched poles/zeros to train the neural networks allows us to have fast and reliable predictions for the poles/zeros subject to large geometrical variations, consequently increasing the accuracy of the overall model.

In the second part of this thesis, we propose a novel surrogate-assisted yielddriven EM optimization approach combining parallel space-mapping (SM), trust region algorithm, and polynomial chaos expansion (PCE). In this approach, a novel trust region algorithm is proposed to increase the robustness of the SM surrogate in each iteration during yield optimization. Moreover, for the first time, parallel computation method is incorporated into SM-based yield-driven design to accelerate the overall yield optimization process of microwave structures. The use of parallel computation allows the surrogate developed in the proposed technique to be valid in a larger neighborhood than that in standard SM, consequently increasing the
\end{abstract}


speed of finding the optimal yield solution in SM-based yield-driven design. Lastly, the PCE approach is incorporated into the proposed technique to further speed up yield verification on the fine model. The proposed technique can achieve a higher yield increase with shorter CPU time by reducing the number of SM iterations.

As a further advancement, in the third part of this thesis, we propose a novel non-surrogate-based approach to yield-driven EM optimization based on PCE. We first formulate a novel objective function for yield-driven EM optimization. By incorporating the PCE coefficients into the formulation, the objective function is analytically related to the nominal point. We then derive the sensitivity formulas of the PCE coefficients with respect to the nominal point, following which we derive the sensitivities of the objective function. These sensitivities are then used in gradientbased optimization algorithms to find the optimal yield solution iteratively. Using the proposed objective function, the number of EM simulations required to find the update direction and suitable step size for the change of the nominal point is reduced at each iteration of optimization. This allows the proposed technique to achieve similar yield increase using much fewer EM simulations or greater yield increase using similar number of EM simulations. All the proposed approaches are illustrated by microwave examples.

KEY WORDS: Artificial neural networks, electromagnetic (EM) parametric modeling, polynomial chaos expansion (PCE), space mapping (SM), yield optimization. 
To my parents and wife 


\section{Acknowledgements}

I would like to express my sincere appreciation to my supervisor, Prof. Q. J. Zhang for the constant support and expert guidance during the course of this research work. His rigorous academic attitude, warmth encouragement, and innovative insights have not only broadened my academic vision but also made my research journey a memorable one. His great support gave me the motivation and direction to march forward on the academic road. It was my great honor to work under his supervision and guidance.

I would also like to express my appreciation to my co-supervisor, Prof. Jianguo Ma for the invaluable counsel and knowledgeable instruction. I would like to thank Prof. Pavan Gunupudi, Prof. Ram Achar, and Prof. Derek McNamara for their valuable suggestions and corrections for my thesis.

Specially, I would like to gratefully acknowledge the members of my Ph.D. thesis examination committee: Prof. Slawomir Koziel, Prof. Emad Gad, Prof. Ram Achar, Prof. Yu Luo, Prof. Qiang Liu, and Prof. Haipeng Fu for their time, invaluable suggestions and corrections.

I would like to thank Tianjin University, where I have stayed for ten years accomplishing both my undergraduate and Ph.D. studies. I would also like to thank Carleton University for the Cotutelle program for my Ph.D. study.

I would like to thank all my present and former colleagues especially Dr. Lin Zhu, Dr. Shuxia Yan, Dr. Feng Feng, Dr. Venu-Madhav-Reddy Gongal-Reddy, Dr. Chao Zhang, Dr. Weicong Na, Dr. Wenyuan Liu, Shunlu Zhang, Chuan Zhang, Kai Ma, Wei Zhang, Zhihao Zhao, Jing Jin, and Jinyuan Cui in our research group 
for their enthusiasm and valuable discussions.

Finally, I wish to thank my parents Yaojun Zhang and Xiangmin Yang, my parents-in-law, my uncle, and my grandparents for their love, belief, support, and encouragement throughout my study. A special thank to my wife Junling Li for her constant accompany, encouragement, and motivation. We have been together for eight years through my undergraduate and Ph.D. studies. Together we have not only enjoyed countless happy times but also overcome many of the challenges through my whole Ph.D. career. 


\section{Table of Contents}

Abstract $\quad$ i

Acknowledgements iv

List of Figures $\quad$ xi

List of Tables $\quad$ xix

1 Introduction $\quad 1$

1.1 Introduction and Motivation . . . . . . . . . . . . . 1

1.2 List of Contributions . . . . . . . . . . . . . . . . 5

1.3 Thesis Organization . . . . . . . . . . . . . 8

2 Literature Review $\quad 10$

2.1 ANN-Based Parametric Modeling Methods . . . . . . . . . . . . . 10

2.2 Neuro-Transfer Function Parametric Modeling Methods . . . . . . . 13

2.2.1 Parametric Modeling of Microwave Components Using Combined Neural Network and Rational Transfer Function . . . 13 
2.2.2 Parametric Modeling of Microwave Components Using Combined Neural Network and Pole-Residue-Based Transfer Func-

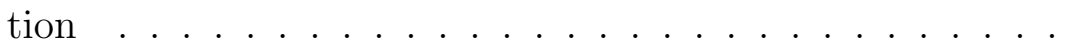

2.2.3 Parametric Modeling of Microwave Components Using Adjoint Neural Networks and Pole-Residue Transfer Functions with EM Sensitivity Analysis . . . . . . . . . . . . 16

2.2.4 Transfer Function Based Parametric Modeling Method Incorporating the Model Order Reduction Technique . . . . . . . 18

2.3 Space Mapping-Based Yield-Driven EM Optimization Methods . . . 19

2.3.1 Yield-Driven EM Optimization Using SM-Based Neuralmodels 19

2.3.2 The Ellipsoidal Technique for Yield-Driven EM Optimization Using Space Mapping Surrogates . . . . . . . . . . . 20

2.3.3 Yield-Driven EM Optimization Using Tuning Space Mapping Surrogates . . . . . . . . . . . . . . . . 22

2.3.4 Yield-Driven EM Optimization Via a Non-Derivative Trust Region Approach and Space Mapping Surrogates . . . . . . 24

2.4 Feature-Based Yield-Driven EM Optimization Methods . . . . . . . 24

2.5 Polynomial Chaos Expansion Based Approach to Statistical Analysis and Yield Estimation of Microwave Circuits . . . . . . . . . . 26

2.5.1 Formulation of Polynomial Chaos Expansion Approach . . . 28

2.5.2 Performing Statistical Analysis and Yield Estimation Using PCE Approach . . . . . . . . . . . . . . . . . . 29

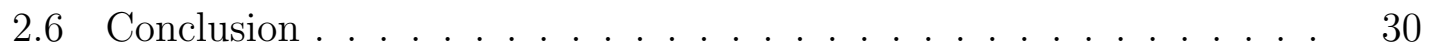


3 Parametric Modeling of Microwave Passive Components Using PVL $\begin{array}{ll}\text { and EM Sensitivities } & 31\end{array}$

3.1 Introduction . . . . . . . . . . . . . . . . 31

3.2 Proposed Training Approach for Parametric Modeling of Microwave Passive Components . . . . . . . . . . . . . . . . . . . 32

3.2.1 The Issue of Mismatch of Poles and Zeros . . . . . . . . 35

3.2.2 Sensitivity-Analysis-Based Pole/Zero-Matching Algorithm . 40

3.2.3 Preliminary Training of the Neural Networks . . . . . . . . 51

3.2.4 Refinement Training of the Proposed Neuro-TF Model . . . 51

3.3 Application Examples _. . . . . . . . . . . . . . 53

3.3.1 Parametric Modeling of a Fifth-Order Waveguide Bandpass Filter: Simple Example with Two Geometrical Variables . . 53

3.3.2 Parametric Modeling of a Three-Pole H-Plane Filter . . . . . 62

3.3.3 Parametric Modeling of the Fifth-Order Waveguide Bandpass Filter: Harder Example with Nine Geometrical Variables . . 69

3.4 Conclusions and Summary . . . . . . . . . . . . . 75

4 Surrogate-Assisted Approach to Yield-Driven EM Optimization 77

4.1 Introduction . . . . . . . . . . . . . . . 77

4.2 Formulation of the Original EM-based Yield Optimization Problem 81

4.3 Proposed Parallel Space-Mapping Based Yield Optimization Technique Incorporating Trust Region Algorithm and PCE $\ldots \ldots$. . . 83 4.3.1 Fine Model Data Generation with Parallel Computation Method 84 
4.3.2 Surrogate Modeling over Multiple Geometrical Samples Using Parallel Computation Method . . . . . . . . . . . 85

4.3.3 The One-Sided $l_{1}$ Centering Algorithm for Yield Optimization Using the Parallel SM Surrogate . . . . . . . . . . . 88

4.3.4 Proposed Trust Region Algorithm for Parallel SM Based Yield Optimization ...................... 90

4.3.5 Incorporating the PCE Approach for Yield Verification on the Fine Model . . . . . . . . . . . . . . . . . . . . . . . . . . 92

4.3.6 The Proposed Yield Optimization Algorithm . . . . . . . . . 95

4.3.7 Discussion . . . . . . . . . . . . . . . . . 96

4.4 Application Examples . . . . . . . . . . . . . . . . . . . . 97

4.4.1 Yield Optimization of a Low-Pass Elliptic Microstrip Filter . 97

4.4.2 Yield Optimization of a Bandstop Microstrip Filter with Open Stubs ......................... 102

5 Non-Surrogate-Based Approach to Yield-Driven EM Optimization $\begin{array}{ll}\text { Exploiting PCE } & 107\end{array}$

5.1 Introduction . . . . . . . . . . . . . . . . . . . 107

5.2 Formulations of the PCE Approach . . . . . . . . . . . . . . . 108

5.3 Proposed Non-Surrogate-Based Yield Optimization Approach Ex-

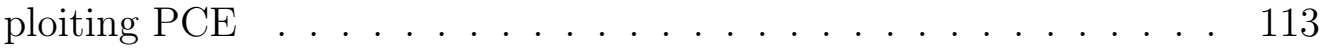

5.3.1 Formulation of the Objective Function for Yield-Driven EM Optimization Incorporating PCE Coefficients . . . . . . . . 114 
5.3.2 Derivation of Sensitivity Formulas for the Proposed Objective Function . . . . . . . . . . . . . . . . . . 122

5.3.3 PCE-based Yield Optimization Algorithm . . . . . . . . . 126

5.4 Examples . . . . . . . . . . . . . . . . . . . . . . 129

5.4.1 Yield Optimization of a Waveguide K-Band Bandpass Filter 129

5.4.2 Yield Optimization of a Waveguide Bandpass Filter with FractalShaped Irises . . . . . . . . . . . . . . . 134

5.4.3 Yield Optimization of a Four-Pole Waveguide Filter . . . . . 139

5.5 Conclusions and Summary . . . . . . . . . . . . . . . . . . 143

6 Conclusions and Future Research 144

6.1 Conclusions . . . . . . . . . . . . . . . . . . . . . 144

6.2 Future Research . . . . . . . . . . . . . . . . . . 146

$\begin{array}{lr}\text { Appendix } & 149\end{array}$

$\begin{array}{ll}\text { Bibliography } & 156\end{array}$ 


\section{List of Figures}

2.1 Structure of the rational function based neuro-TF model [17]. . . . 14

2.2 Structure of the pole-residue-based neuro-TF model, from [20]. $\boldsymbol{x}$ represents the vector of geometrical variables. $\boldsymbol{y}$ represents the output vector of the model. $\boldsymbol{d}$ represents the desired outputs of the model. ....................... . . 16

2.3 Structure of the sensitivity-analysis-based neuro-TF model [20]. $\boldsymbol{x}$ represents the vector of geometrical variables, $\boldsymbol{y}$ represents the output vector of the transfer function, and $d \boldsymbol{y} / d \boldsymbol{x}$ denotes the derivatives of $\boldsymbol{y}$ with respect to $\boldsymbol{x} . \ldots \ldots \ldots \ldots$

2.4 An illustration of the yield optimization procedure using SM-based neuralmodels. . . . . . . . . . . . . . . . . . .

2.5 A summary of the yield optimization framework using the SM-based ellipsoidal technique. . . . . . . . . . . . . . . . .

2.6 An illustration of the yield optimization procedure using tuning SM surrogates. . . . . . . . . . . . . . . . 
2.7 An illustration of the overall yield optimization process using the NEWUOA algorithm and SM surrogates. . . . . . . . . . . . .

2.8 An illustration of the overall yield optimization process using the feature-based method presented in $[32] \ldots \ldots \ldots$. . . . . .

3.1 Structure of the proposed neuro-TF model. $\boldsymbol{x}$ represents the geometrical variables. $\boldsymbol{y}$ represents the outputs (e.g., $S$-parameters) of the transfer function in the complex propagation space. $\boldsymbol{d}$ represents the outputs of EM simulations. . . . . . . . . . . . . .

3.2 Illustration of the mismatch issue of poles w.r.t. one geometrical variable using a fifth-order waveguide bandpass filter example: (a) The original unsorted poles obtained directly from the eigendecomposition processes; (b) The poles sorted according to their magnitudes; (c) Correct sorting results of the two poles. In both (a) and (b), the dashed circles indicate the areas where the issue of mismatch of poles occurs. . . . . . . . . . . . . . . .

3.3 Flow chart of the proposed sensitivity-analysis-based pole-matching algorithm to match the poles between two neighboring geometrical parameter values, i.e., between $\boldsymbol{x}^{(k)}$ and $\boldsymbol{x}^{(l)} \ldots \ldots$. . . . . . 
3.4 Flow chart of the algorithm to enumerate all the scattered geometrical parameter samples into pairs and to organize the sequence of pairs for matching the poles following a common set of reference indices for all the geometrical samples. The details in the dashed block is described by Fig. 3.3. . . . . . . . . . . . . . .

3.5 Mechanism for the refinement training process of the overall model. The optimization variables here are the weighting parameters $\boldsymbol{w}_{p}$, $\boldsymbol{w}_{z}$, and $\boldsymbol{w}_{K}$ in neural networks $p_{N N}, z_{N N}$, and $K_{N N} \ldots \ldots$

3.6 Flow chart of the overall training approach for developing parametric models of microwave passive components. . . . . . . . . . . .

3.7 Structure of the fifth-order waveguide bandpass filter for EM simulation and parametric modeling: Simple example with two geometrical variables. For this simple example, only $d_{1}$ and $d_{2}$ are geometrical variables as inputs to the model, i.e., $\boldsymbol{x}=\left[\begin{array}{ll}d_{1} & d_{2}\end{array}\right]^{T} \ldots . . . .$.

3.8 Original distributions of the three selected poles $\left(p_{1}, p_{2}\right.$, and $\left.p_{4}\right)$ in the " $\gamma$-space". These poles are evaluated using MPVL at 49 geometrical parameter samples in the training data for Case 2 of the fifth-order waveguide bandpass filter. There are a total of 147 poles (three groups with each group having 49 poles) in this figure. From these unsorted poles, we need to use a pole-matching method to identify which 49 poles belong to $p_{1}$, which 49 poles belong to $p_{2}$, and which 49 poles belong to $p_{4}$, by sorting the poles at each of the 49 geometrical parameter values correctly. . . . . . . . . . . 
3.9 Pole-matching solutions of the three selected poles with different methods in Case 2 of the fifth-order waveguide bandpass filter example. The figure shows how the 147 poles in Fig. 3.8 are sorted and grouped into $p_{1}$ (solid squares), $p_{2}$ (circles), and $p_{4}$ (crosses) using different pole-matching methods: (a) The proposed sensitivity-analysisbased pole-matching algorithm, (b) The distance-based method, and (c) The continuation method. It is seen that different methods have different pole-matching solutions. . . . . . . . . . . . .

3.10 Detailed pole-matching solution for $p_{1}$ showing the degree of similarity of the patterns between training samples and testing samples for the fifth-order waveguide bandpass filter example: (a) The proposed sensitivity-analysis-based pole-matching algorithm, (b) The distancebased method, and (c) The continuation method. The training and testing samples of $p_{1}$ are represented by hollow and solid circles, respectively. . . . . . . . . . . . . . . . .

3.11 Structure of the three-pole $H$-plane filter for EM simulation and parametric modeling. The four geometrical parameters of the filter are $\boldsymbol{x}=\left[\begin{array}{llll}L_{1} & L_{2} & W_{1} & W_{2}\end{array}\right]^{T}$, with $a=19.05 \mathrm{~mm}, b=9.525 \mathrm{~mm}$, and $t=2.0$

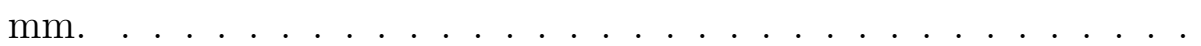

3.12 Structure of the neuro-TF model for the three-pole $H$-plane filter example. . . . . . . . . . . . . . . . . . 
3.13 Comparison of the outputs ( $\left|S_{11}\right|$ in decibels) of the models and the EM data for Case 2 of the three-pole $H$-plane filter example: (a) Test geometrical parameter value \#1, (b) Test geometrical parameter value \#2, and (c) Test geometrical parameter value \#3. The three existing methods are: 1) Distance-based method, 2) Distance-based method with refinement training, and 3) Continuation method with refinement training. The model outputs obtained from the proposed approach matches the EM data much better than the model outputs obtained from the three existing methods. . . . . . . . . . . . . . 68

3.14 Structure of fifth-order waveguide bandpass filter for EM simulation and parametric modeling: Harder example with nine geometrical variables. The nine geometrical variables of the filter are $\boldsymbol{x}=$

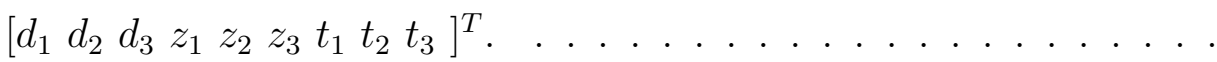

3.15 Structure of the neuro-TF model for the fifth-order waveguide bandpass filter example with nine geometrical variables. . . . . . . . . . 70 
3.16 Comparison of the outputs ( $\left|S_{11}\right|$ in decibels) of the models and the EM data for Case 2 of the fifth-order waveguide bandpass filter example: (a) Test geometrical parameter value \#1, (b) Test geometrical parameter value \#2, and (c) Test geometrical parameter value \#3. The three existing methods are: 1) Distance-based method, 2) Distance-based method with refinement training, and 3) Continuation method with refinement training. It can be seen that the model outputs obtained from the proposed approach matches with the EM data much better than the model outputs obtained from the three existing methods. . . . . . . . . . . . . . . . . 72

4.1 Flow chart of the proposed yield optimization technique. . . . . . . 93

4.2 The structure of the low-pass elliptic microstrip filter used in EM simulation and yield optimization, from [35]. . . . . . . . . . . . 98

4.3 Yield optimization results of the low-pass microstrip filter using the proposed technique: (a) Before and (b) After yield optimization. In both figures, the grey dashed lines indicate 100 random samples in Monte Carlo analysis, while the black solid line indicates the nominal response. . . . . . . . . . . . . . . . . . . . . 101

4.4 The structure of the bandstop microstrip filter defined for EM simulation and yield optimization, from [35]. . . . . . . . . . . . . 103 
4.5 Yield optimization results of the bandstop microstrip filter using the proposed technique: (a) Before and (b) After yield optimization. In both figures, the grey dashed lines indicate 100 random samples in Monte Carlo analysis, while the black solid line indicates the nominal response. . . . . . . . . . . . . . . . . 106

5.1 Illustration of the movement of sparse grid samples in PCE between two consecutive iterations during yield optimization. The two black dots represent the nominal points between two successive iterations. The circles represent the integration samples around the nominal points following the sparse grid technique. In yield optimization, the nominal point $\boldsymbol{x}^{0}$ is a variable which is updated iteratively. As the nominal point moves from $\boldsymbol{x}^{0}$ to $\boldsymbol{x}_{\text {new }}^{0}$, all the sparse grid samples move accordingly. . . . . . . . . . . . . . . . . . . . . . 124

5.2 Flow chart of the proposed PCE-based yield optimization algorithm. 129

5.3 The structure of the K-band bandpass filter example for EM simulation and yield optimization. The three design parameters of the filter are $\boldsymbol{x}=\left[r_{1} r_{2} d\right]^{T} \ldots \ldots \ldots \ldots \ldots \ldots$

5.4 Yield optimization results of the K-band bandpass filter: (a) Before yield optimization and (b) After yield optimization. For both figures, grey dashed lines indicate 100 samples from Monte Carlo analysis, while the black solid line indicates the response evaluated at the nominal point. . . . . . . . . . . . . . . 
5.5 The structure of the waveguide bandpass filter example with fractalshaped irises for EM simulation and yield optimization. The three design parameters of the filter are $\boldsymbol{x}=\left[\begin{array}{lll}d_{1} & d_{2} & d_{3}\end{array}\right]^{T} \ldots \ldots$. . . . . . 136

5.6 Details of the geometrical structure of the fractal shaped iris as circled in Fig. 5.5. . . . . . . . . . . . . . . . . . . . . . . 136

5.7 Yield optimization results of the waveguide bandpass filter with fractalshaped irises: (a) Before yield optimization and (b) After yield optimization. Grey dashed lines indicate 100 samples from Monte Carlo analysis, while the black solid line indicates the response evaluated at the nominal point. . . . . . . . . . . . . . . . . 138

5.8 The structure of the four-pole waveguide filter example for EM simulation and yield optimization. The five design parameters of the filter are $\boldsymbol{x}=\left[\begin{array}{ll}\left.h_{1} h_{2} h_{3} h_{c 1} h_{c 2}\right]^{T} \ldots \ldots \ldots \ldots \ldots & \ldots \ldots \ldots \ldots\end{array}\right.$

5.9 Yield optimization results of the four-pole waveguide filter: (a) Before and (b) After yield optimization. Grey dashed lines indicate 300 samples from Monte Carlo analysis, while the black solid line indicates the response evaluated at the nominal point. . . . . . . . . 141 


\section{List of Tables}

3.1 The Original Unsorted Poles Versus Changing Values of the Geometrical Parameter $L$ - An Illustrative Example . . . . . . . . . . . 36

3.2 The Poles of Table 3.1 After Being Sorted Correctly . . . . . . . 36

3.3 Definition of Training and Testing Data for the Fifth-Order Bandpass Filter Example with Two Geometrical Variables . . . . . . . . . . 55

3.4 Model Accuracy Comparisons of Different Modeling Methods for the Fifth-Order Bandpass Filter Example with Two Geometrical Variables 61

3.5 Definition of Training and Testing Data for the Three-Pole $H$-Plane Filter Example . . . . . . . . . . . . . . . . . 65

3.6 Model Accuracy Comparisons of Different Modeling Methods for the Three-Pole $H$-Plane Filter Example . . . . . . . . . . . . . . 66

3.7 Definition of Training and Testing Data for the Fifth-Order Bandpass Filter Example with Nine Geometrical Variables . . . . . . . . . 73

3.8 Model Accuracy Comparisons of Different Modeling Methods for the Fifth-Order Bandpass Filter Example with Nine Geometrical Variables 74 
4.1 Comparison of Standard SM based Yield Optimization Technique and the Proposed Yield Optimization Technique for the Lowpass Microstrip Filter . . . . . . . . . . . . . . . 100

4.2 Comparison of Standard SM based Yield Optimization Technique and the Proposed Yield Optimization Technique for the Bandstop Microstrip Filter . . . . . . . . . . . . . . . . . 104

5.1 Comparison of Yield Optimization Results for the K-Band Bandpass

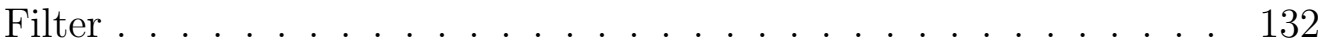

5.2 Comparison of Yield Improvements With Similar Number of EM Simulations for the K-Band Bandpass Filter . . . . . . . . . . . . 133

5.3 Comparison of Yield Optimization Results for the Waveguide FSI Filter 137

5.4 Comparison of Yield Improvements With Similar Number of EM Simulations for the Waveguide FSI Filter . . . . . . . . . . . . . . . 139

5.5 Comparison of Yield Optimization Results for the Four-Pole Waveguide Filter . . . . . . . . . . . . . . . . . . . . . . . . . . . 142

5.6 Comparison of Yield Improvements With Similar Number of EM Simulations for the Four-Pole Waveguide Filter . . . . . . . . . . . . . . 143 


\section{List of Symbols}

$a_{i j}$ The PCE coefficient for the $i$-th basis function $\Phi_{i}$ of the $j$-th EM response

$b_{i}$ The problem-independent constant for the $i$-th basis function $\Phi_{i}$

$c$ The constant in the transfer function in the propagation constant space

$\boldsymbol{D}$ The matrix containing the distances between the actual and the predicted new positions of the poles at $\boldsymbol{x}^{(l)}$

$D$ The highest polynomial order in the polynomial chaos expansion

$\boldsymbol{d}$ The EM responses (e.g., magnitudes of $S$-parameters) from EM simulations

$\boldsymbol{d}^{(n)}$ The EM responses (e.g., magnitudes of $S$-parameters) evaluated at $\boldsymbol{x}^{(n)}$

$E\left(R_{j}\right)$ The mean value of the EM response $R_{j}$

$E_{t r}$ The error function to be minimized when training the proposed neuro-TF model

$E(\boldsymbol{w})$ The error function to be minimized when training the SM surrogate model 
$\boldsymbol{e}(\boldsymbol{x})$ The error vector defined to measure the degree to which the response satisfies the design specifications

$e_{j}$ The $j$ th element in the error vector $\boldsymbol{e}(\boldsymbol{x})$

$f\left(\xi_{d}\right)$ The probability density function of $\xi_{d}$

$f\left(R_{j}\right)$ The probability density function of EM response $R_{j}$

$H(\omega)$ The frequency response (e.g., $\left.S_{11}\right)$ of microwave passive components

$H_{p}(\cdot)$ The one-sided least $p$-th function

$i_{r}$ The row index of the minimum element in $\boldsymbol{D}$ in the $r$ th round

$\boldsymbol{J}_{p}^{(n)}$ The matrix containing the EM sensitivities of poles w.r.t. geometrical variables at $\boldsymbol{x}^{(n)}$

$\boldsymbol{J}_{z}^{(n)}$ The matrix containing the EM sensitivities of zeros w.r.t. geometrical variables at $\boldsymbol{x}^{(n)}$

$\bar{J}$ The set containing all the indices of the specification samples which are violated by at least one outcome

$\bar{J}_{l}$ contain the indices of the lower specification samples which are violated by at least one outcome

$\bar{J}_{u}$ contain the indices of the upper specification samples which are violated by at least one outcome 
$j_{r}$ The column index of the minimum element in $\boldsymbol{D}$ in the $r$ th round

$\bar{K}_{j}$ The set containing all the indices of the outcomes whose responses violate the specification sample $S_{j}$

$K$ The gain of the transfer function in the complex propagation space

$K_{N N}$ The outputs of the neural networks for gain

$K^{(n)}$ The gain evaluated at the geometrical sample $\boldsymbol{x}^{(n)}$

$M$ The total number of integration quadrature points in the sparse grid technique

$m$ The number of specification samples, including samples for upper specifications and samples for lower specifications

$m_{l}$ The number of lower specification samples

$m_{u}$ The number of upper specification samples

$N$ The total number random outcomes of $\boldsymbol{x}$

$N_{\text {in }}$ The number of unknowns on the input port

$N_{\text {iter }}$ The iteration counter in polynomial chaos based yield optimization

$N_{i t e r}^{\max }$ The maximum iteration count in polynomial chaos based yield optimization

$N_{j}^{f a i l}$ The number of elements in $\bar{K}_{j}$

$N_{s}$ The total number of design specification samples 
$N_{s}^{u}$ The number of upper specification samples

$N_{s}^{l}$ The number of lower specification samples

$n$ The dimension of geometrical parameters $\boldsymbol{x}$

$P$ The number of terms in polynomial chaos expansion minus 1

$\boldsymbol{p}$ The vector containing all the poles in $H(\omega)$ at a given geometrical sample

$\boldsymbol{p}^{(n)}$ The vector containing the poles evaluated at $\boldsymbol{x}^{(n)}$

$\overline{\boldsymbol{p}}^{(l)}$ The predicted new positions of the poles at $\boldsymbol{x}^{(l)}$

$\bar{p}_{i}^{(l)}$ The predicted new position for the $i$ th pole at $\boldsymbol{x}^{(l)}$

$p_{j}^{(l)}$ The actual new position for the $j$ th pole at $\boldsymbol{x}^{(l)}$

$p_{N N}$ The outputs of the neural networks for the poles

$q$ The order of reduced-order models and also the number of poles in the transfer function in the " $\gamma$-space"

$R_{j}(\boldsymbol{x})$ The EM response at the frequency of interest that corresponds to $S_{j}$

$R_{j}\left(T^{-1}\left(\boldsymbol{x}^{0}, \boldsymbol{\xi}\right)\right)$ The EM response at the frequency of interest that corresponds to $S_{j}$, with $\boldsymbol{x}^{0}$ being explicitly presented

$\boldsymbol{R}^{c}(\boldsymbol{x})$ The response vector of the coarse model

$\boldsymbol{R}^{f}(\boldsymbol{x})$ The response vector of the fine model 
$\boldsymbol{R}^{s}(\boldsymbol{x}, \boldsymbol{w})$ The response vector of the SM surrogate

$S$ The set containing all the pairs of $\boldsymbol{u}$ and $\boldsymbol{v}$

$S_{j}$ The $j$-th design specification sample, where $j=1, \cdots, m$

$S_{p}$ The parallel speedup factor

$\boldsymbol{T}$ The complex-valued matrix reduced from the original system matrix in FEM

$T\left(\boldsymbol{x}^{0}, \boldsymbol{x}\right)$ The transformation to transform the original random parameters $\boldsymbol{x}$ to independent standard random parameters $\boldsymbol{\xi}$

$T^{-1}\left(\boldsymbol{x}^{0}, \boldsymbol{\xi}\right)$ The inverse transformation corresponding to $T$

$T_{r}$ The set containing the indices of the training samples at different geometrical parameter values

$U\left(\boldsymbol{x}^{0}\right)$ The objective function in traditional Monte Carlo-based yield optimization approach

$\bar{U}\left(\boldsymbol{x}^{0}\right)$ The new objective function incorporating PCE coefficients for yield-driven EM optimization

$\bar{u}_{j}\left(\boldsymbol{x}^{0}\right)$ The yield indicator with respect to the specification sample $S_{j}$

$\boldsymbol{u}$ A sample of $\boldsymbol{x}$ from $\Gamma$

$V_{j}\left(\boldsymbol{x}^{0}\right)$ The first part of the new objective function incorporating PCE coefficients for yield-driven EM optimization 
$\boldsymbol{v}$ A sample of $\boldsymbol{x}$ from $\Upsilon$

$\boldsymbol{w}$ The neural network weights

$\boldsymbol{w}_{K}$ The internal neural network weights corresponding to $K_{N N}$

$\boldsymbol{w}_{p}$ The internal neural network weights corresponding to $p_{N N}$

$\boldsymbol{w}_{z}$ The internal neural network weights corresponding to $z_{N N}$

$w^{(l)}$ The $l$ th integration quadrature weight corresponding to $\boldsymbol{\xi}^{(l)}$

$\mathcal{X}$ The set of EM design parameters generated around the current nominal point $\boldsymbol{x}^{0}$ following the sparse grid technique

$X$ The set containing all the geometrical parameter values in the training data

$\boldsymbol{x}$ The vector of design parameters (e.g., geometrical parameters) of the EM structure

$\boldsymbol{x}_{c}$ The vector containing all the design variables of the coarse model

$\boldsymbol{x}_{f}$ The vector containing all the design variables of the fine model

$\boldsymbol{x}^{0}$ The nominal point of $\boldsymbol{x}$

$\boldsymbol{x}^{0, k}$ The nominal point at the $k$ th iteration during SM-based yield optimization

$\boldsymbol{x}_{n e w}^{0}$ The new nominal point during yield optimization

$\boldsymbol{x}^{k}$ The $k$ th random outcome of $\boldsymbol{x}$ 
$\boldsymbol{x}^{(l)}$ The $l$ th sparse grid sample in the design parameter space

$\boldsymbol{x}^{*}$ The final optimal yield solution

$\boldsymbol{y}$ The output vector of the proposed neuro-TF model

$Y_{t h}$ The user-defined criteria to terminate the overall yield optimization process

$z_{N N}$ The outputs of the neural networks for the zeros

$\gamma(\omega)$ The complex propagation constant

$\gamma_{0}$ The expansion point in frequency

$\delta$ The threshold for a neighborhood

$\boldsymbol{\delta}^{k}$ The trust radii for the design variables in the $k$ th iteration

$\boldsymbol{\Delta}_{\max }$ The maximal allowed trust radii for the design variables

$\varepsilon$ The user-defined threshold to terminate the PCE-based yield optimization

$\eta$ The parallel efficiency

$\boldsymbol{\eta}$ A $q \times N_{\text {in }}$ complex-valued matrix returned from the MPVL algorithm

$\boldsymbol{\xi}$ The vector of independent standard random parameters

$\boldsymbol{\xi}^{(l)}$ The $l$ th integration quadrature point in the " $\boldsymbol{\xi}$-space"

$\boldsymbol{\rho}$ A $q \times 1$ complex-valued column matrix returned from the MPVL algorithm $\rho(\boldsymbol{\xi})$ The joint probability density function of the standard random parameters $\boldsymbol{\xi}$ xxvii 
$\sigma_{d}$ The standard deviation for the $d$-th design parameter $x_{d}$

$\phi(\cdot)$ The cumulative distribution function of the standard normal distribution

$\omega$ The angular frequency

$\Gamma$ The set containing the geometrical parameter values at which the poles are already matched

$\Delta \boldsymbol{x}$ The change of geometrical parameters between $\boldsymbol{x}^{(l)}$ and $\boldsymbol{x}^{(k)}$

$\Upsilon$ The set containing the geometrical parameter values at which the poles are not matched yet

$\Phi$ The final pole-matching solution between $\boldsymbol{x}^{(l)}$ and $\boldsymbol{x}^{(k)}$

$\Phi_{i}(\cdot)$ The generalized polynomial chaos basis function

$\Omega^{k}$ The trust region of the SM surrogate model in iteration $k$

$\Omega^{n}$ The $n$-dimensional space of all possible values of $\boldsymbol{\xi}$

$\Omega_{1}^{r}$ The set containing all the row indices of the minimum elements found in the rounds prior to round $r$

$\Omega_{2}^{r}$ The set containing all the column indices of the minimum elements found in the rounds prior to round $r$

$\|\cdot\| L_{2}$ norm 


\section{Chapter 1}

\section{Introduction}

\subsection{Introduction and Motivation}

Microwave passive components, such as microwave filters and antennas, are widely used in today's smart phones, laptops, and many other electronic devices. To reach the optimal design of a microwave passive component, three-dimensional electromagnetic (EM) simulations are typically needed to simulate the EM behavior of the component, and an optimization process has to be performed to obtain the optimal set of physical dimensions. EM design optimization require parametric studies where the given EM structure has to be analyzed at different geometrical parameter values. This process is computationally expensive as repetitive EM simulations with different geometrical parameter values have to be performed. Parametric modeling techniques hold a great potential to accelerate this process. Typically, parametric models are developed to represent the EM behavior of microwave components subject to geometrical variations. Once developed, the models can provide fast and accurate prediction of the EM behavior of microwave passive components, and can 
be further utilized in high-level microwave circuit and system designs [1]-[4].

Artificial neural networks (ANNs) are recognized vehicles in EM parametric modeling and design optimization [1], [3]-[10]. Through a suitable training process, ANNs can represent the relationship between the EM responses and geometrical parameters. To improve the accuracy and reliability of ANN parametric modeling, knowledge-based neural network models have been developed. The knowledge-based approaches combine neural networks with prior knowledge such as equivalent circuits [11]-[13] and analytical expressions [14], [15]. These knowledge are used to help improve the capability of learning and generalization of the overall models as well as to accelerate the model development process [16].

Another advanced EM parametric modeling approach, i.e., the neuro-transfer function (neuro-TF) modeling approach, has been reported in [17]-[21]. This approach can be used even if accurate equivalent circuit models are not available. With this method, the EM behavior of microwave passive components versus frequency can be expressed by transfer functions in a certain form. Since the coefficients (or poles/residues) in the transfer functions change as geometrical parameters change, neural networks are typically trained to learn the relationship between the coefficients (or poles/residues) in the transfer functions and geometrical parameters. The coefficients (or poles/residues) in transfer functions corresponding to a given set of EM responses, are obtained by the vector fitting technique [22].

Recently, another alternative transfer function based parametric modeling method that does not rely on the use of the vector fitting technique has been presented in [23] and [24]. The transfer functions used in this alternative method are derived from 
an application of the model-order reduction (MOR) technique. The poles/residues in the transfer function are obtained directly from full-wave EM simulations instead of through a vector fitting process. Thanks to the MOR technique, the order of transfer function between different geometrical parameter values can be consistent. Therefore, one can use a constant order for the transfer function at different geometrical parameter values to avoid the order-changing problem in traditional neuro-TF modeling methods.

This group of methods addressed a common problem, i.e., the problem of mismatch of poles. To estimate the poles for a new change of geometrical parameters, interpolations are needed based on existing samples of poles. These samples are computed (or recomputed) by the MOR technique each time we change the geometrical parameters. However, the indices of the poles after the recomputation may not have clear correspondences with those before the recomputation. This may lead to wrong predictions of the poles for a new change of geometrical parameters. An intuitive distance-based algorithm has been presented in [23] to address this problem. As a further extension, an extrapolation based continuation method has been presented in [24] to deal with the combinatorial explosion issue and improve the solution quality while matching the poles. These two methods both perform well in applications with small/medium geometrical variations. However, how to address the issue of mismatch of poles to achieve accurate parametric models for more challenging applications involving large geometrical variations still remains an open subject in the literature.

Furthermore, uncertainties introduced in the manufacturing process pose in- 
herent randomness on physical dimensions and material properties of microwave components. Under this consideration, performing yield-driven EM optimization is an essential step in manufacturability-driven designs of microwave passive components [25], [26]. Many techniques have been developed for yield-driven EM yield optimization in the last two decades. One group of methods are the space-mapping (SM) based methods such as those in [27]-[30]. Another group of methods are the feature-based methods such as those in [31] and [32].

All the aforementioned SM-based methods do not use parallel computation, which holds a great potential to speed up EM design optimization. Parallel computation is an efficient technique to speed up EM optimization by accelerating EM data generation [33] and surrogate model training [34]. Recently, a parallel SM approach has been presented in [35], where the parallel computation mechanism has been combined with SM, to facilitate EM-based nominal optimization. It has been shown that with parallel computation, the SM surrogate can be trained to be valid in a relatively larger neighborhood and the optimal nominal design can be obtained in shorter time and fewer SM iterations [35].

At each iteration of yield optimization, the prediction of yield values (also referred to as yield estimation) is typically involved. Recently, approaches based on polynomial chaos expansion (PCE) [36] have emerged as favorable alternatives for yield estimation and statistical analysis in the microwave area, such as [37]-[47]. It has been demonstrated that the PCE approach shows significant computational advantages over the traditional Monte Carlo analysis and can be regarded as a powerful tool in yield estimation and statistical analysis of microwave structures. 
Compared to yield estimation, yield optimization has additional challenges. Unlike yield estimation where one fixed nominal point (usually regarded as the mean values of statistical parameters) is considered, in yield optimization the nominal point is a variable which is updated iteratively, resulting in many nominal points to be considered. At each iteration of yield optimization, yield estimation needs to be performed w.r.t. one nominal point, and the update direction and suitable step size for the change of the nominal point need to be determined. These tasks have to be done repetitively from iteration to iteration during optimization, requiring a large number of EM simulations if we directly apply the conventional Monte Carlo-based or geometrical yield optimization approaches. Considering the computational advantages that $\mathrm{PCE}$ offers in yield estimation, it is of great interest to explore the use of PCE for yield optimization. However, how to exploit the parallel computation method and PCE to facilitate EM-based yield optimization of microwave structures still remains an open subject in the literature.

\subsection{List of Contributions}

The main objective of this thesis is to investigate and develop advanced parametric modeling and yield optimization approaches for microwave passive components. Toward this objective, the following significant contributions are made in this thesis:

- A novel training approach is proposed for developing parametric models of microwave passive components with respect to changes in geometrical parameters using Padé via Lanczos and EM Sensitivities. The EM responses (e.g., $S$-parameters) of passive components versus frequency are represented 
by pole-zero-gain transfer functions. Neural networks are trained to learn the relationship between the poles/zeros/gain in the transfer function and geometrical parameters. To address the issue of mismatch of pole/zeros when large geometrical variations are considered, we propose a novel sensitivity-analysisbased pole/zero-matching algorithm to obtain the correct correspondences between the pole/zeros at different geometrical parameter values. Using the matched poles/zeros to train the neural networks allows us to have fast and reliable predictions for the poles/zeros subject to large geometrical variations, consequently increasing the accuracy and robustness of the overall model. Once developed, the model can provide fast and accurate prediction of the EM behavior of microwave components with geometrical parameters as variables, and can be further utilized in high-level microwave circuit and system designs.

- We propose a surrogate-assisted approach that combines parallel SM, trust region algorithm and PCE to solve the problem of yield-driven EM optimization. A novel trust region algorithm specifically for EM-based yield-driven design is proposed to increase the robustness of the SM surrogate. For the first time, we incorporate the parallel computation method to SM-based yield optimization of microwave structures. The use of parallel computation allows the surrogate in SM to be trained in a larger neighborhood in the design parameter space than that in the standard SM, consequently increasing the speed of finding the optimal yield solution in yield-driven design. The PCE approach is used in the verification of the fine model yield to further accelerate the proposed 
yield optimization technique.

- A PCE-based non-surrogate approach is proposed to solve the problem of yield-driven EM optimization when equivalent circuit coarse models are not available. For the first time, we elevate the use of PCE approach from EMbased yield estimation to EM-based yield optimization. We formulate a novel objective function for yield-driven EM optimization incorporating PCE coefficients. In this way, the objective function is analytically related to the nominal point. The proposed objective function requires fewer EM simulations to provide reliable yield representation than that in the conventional Monte Carlo based yield optimization approach. As a result, the number of EM simulations required to find the update direction and proper step size for the change of the nominal point is reduced at each iteration of optimization. This allows the proposed approach to achieve similar yield increase using much fewer EM simulations or greater yield increase using similar number of EM simulations.

- We derive the sensitivity formulas of the PCE coefficients w.r.t. the nominal point. The sensitivities of the objective function w.r.t. the nominal point are derived based on the sensitivity formulas of the PCE coefficients. These sensitivities are then used in gradient-based optimization algorithms to find the optimal yield solution iteratively. To the best of our knowledge, the proposed PCE-based non-surrogate approach is the first work that provides systematic formulation and sensitivity formulas of EM-based yield optimization, and does not require the availability of a coarse model. 


\subsection{Thesis Organization}

The rest of the thesis is organized as follows: Chapter 2 presents a literature review of popularly used parametric modeling and yield optimization techniques for microwave passive components. First, an overview of ANN-based EM parametric modeling methods and recently reported neuro-transfer function parametric modeling methods are provided. Then, some existing yield-driven EM optimization approaches, including the SM-based methods and the feature-based methods are reviewed. Finally, the PCE-based approaches to statistical analysis and yield estimation of microwave circuits are discussed.

Chapter 3 presents a novel training approach for developing parametric models of microwave passive components with respect to changes in geometrical parameters. The proposed approach addresses the condition that no coarse model is available, and obtain better model accuracy in challenging applications involving large geometrical variations. Three application examples are presented to demonstrate this proposed approach.

Chapter 4 presents a novel parallel SM based yield-driven EM optimization technique incorporating trust region algorithm and PCE. By incorporating the parallel computation method and PCE, the proposed technique can greatly accelerate the overall SM-based yield optimization process by reducing the number of SM iterations. This proposed technique is illustrated by two microwave examples.

Chapter 5 presents a novel PCE-based approach that addresses the yield-driven EM optimization problem when equivalent circuit models are not available. By 
formulating a new objective function incorporating the PCE coefficients, the proposed approach can achieve similar yield increase using much fewer EM simulations or greater yield increase using similar number of EM simulations. This proposed approach is demonstrated by three microwave examples.

Finally, Chapter 6 presents the conclusions and possible future research directions. 


\section{Chapter 2}

\section{Literature Review}

Electromagnetic design optimization can be computationally expensive since it usually requires repetitive EM simulations where the given EM structure has to be analyzed at different geometrical parameter values. Parametric modeling techniques hold a great potential to accelerate EM design optimization. Typically, parametric models are developed to represent the EM behavior of microwave components subject to geometrical variations. Once developed, the models can provide fast and accurate prediction of the EM behavior of microwave passive components, and can be further utilized in high-level microwave circuit and system designs.

\subsection{ANN-Based Parametric Modeling Methods}

Artificial neural networks have been recognized as powerful tools in EM parametric modeling and design optimization [1], [3]-[10]. Through a suitable training process, ANNs can represent the relationship between the EM responses and geometrical parameters. Once trained, the ANN models can provide fast and accurate prediction of the EM behavior of passive components, and can be further utilized in subsequent 
high-level microwave circuit designs.

The past three decades have witnessed the use of ANNs in parametric modeling of a large variety of microwave applications, including microstrip filters [13], [51], antennas [52], vias [53], power amplifiers [5], high-speed interconnects [54], [55], high electron mobility transistor (HEMT) devices [8], transmission line components [56], [57], coplanar waveguide (CPW) components [58], waveguide filters [9], [34], [59], embedded passives [60], [61], voltage-controlled oscillators [7], mixers [62], bends [63], and spiral conductors [64] etc.

Recent research efforts of ANN-based EM parametric modeling techniques have focused on automated model generation (AMG) methods [13], [51], [65], hybrid training methods incorporating parallel processing [34], and multiphysics parametric modeling [66], [67]. In [51], an advanced algorithm for AMG using neural networks has been presented, where interpolation techniques are incorporated to avoid redundant training in AMG, accelerating the overall model generation process. As an extension of the work in [51], an enhanced AMG algorithm has been presented in [13] to automate the development process of knowledge-based neural network models for microwave applications. As a further advance, in [65], the parallel computation method has been incorporated into the AMG algorithm to achieve an additional speedup for neural modeling of microwave devices. In [34], a global neural network training method that combines hybrid training algorithm with parallel processing has been provided, where multiple neural network trainings are distributed to different processors and local search is performed in parallel to increase the probability and speed of finding a global optimum. More recently, ANNs have been introduced 
to multiphysics parametric modeling of microwave components. In [66], neural networks have been applied to multiphysics parametric modeling of microwave filters. In [67], the space mapping (SM) concept has been elevated from pure EM parametric modeling to multiphysics parametric modeling. The coarse model in SM is represented by ANNs trained from the data generated by EM single-physics simulation, while the fine model corresponds to EM-centric multiphysics simulation.

On the other hand, different microwave applications have motivated the research community to investigate and develop ANN-based parametric modeling techniques based on a great variety of ANN structures, such as multilayer perception (MLP) neural networks [68], dynamic neural networks (DNNs) [69], [70], radial basis function (RBF) neural networks [71], [72], recurrent neural networks (RNNs) [73], [74], [75], time-delay neural networks (TDNNs) [76], state-space dynamic neural networks (SSDNNs) [77], [78], and the recently introduced deep neural networks [79].

To enhance the reliability and accuracy of ANN parametric modeling for microwave components, the knowledge-based neural network (KBNN) models have been developed. The knowledge-based approaches combine neural networks with prior knowledge such as equivalent circuits [11]-[13] and analytical expressions [14], [15]. These knowledge are used to help improve the capability of learning and generalization of the overall models as well as to accelerate the model development process [16]. 


\subsection{Neuro-Transfer Function Parametric Model- ing Methods}

Recently, another advanced EM parametric modeling approach, i.e., the neurotransfer function (neuro-TF) modeling approach, has been reported [17]-[21]. This approach can be used even if accurate equivalent circuit models are not available. With this method, the EM behavior of passive components versus frequency can be expressed by transfer functions in a certain form. Since the coefficients (or pole/residues) in the transfer functions change as geometrical parameters change, neural networks are trained to learn the relationship between the coefficients (or

pole/residues) in the transfer functions and geometrical parameters. In the following sections, we review several seminal research works focused on the development of neuro-TF parametric modeling methods.

\subsubsection{Parametric Modeling of Microwave Components Us- ing Combined Neural Network and Rational Transfer Function}

In [17], a rational transfer function based training method for developing parametric neuro-TF models of microwave passive components has been presented. The problem of discontinuity of coefficients in transfer functions, resulting from varied orders of transfer functions at different regions of geometrical parameter space, is also addressed. Figure 2.1 illustrates the structure of the neuro-TF model presented in [17]. As shown in the figure, the model consists of neural network and rational transfer functions. The inputs to the model are geometrical variables and frequency, 


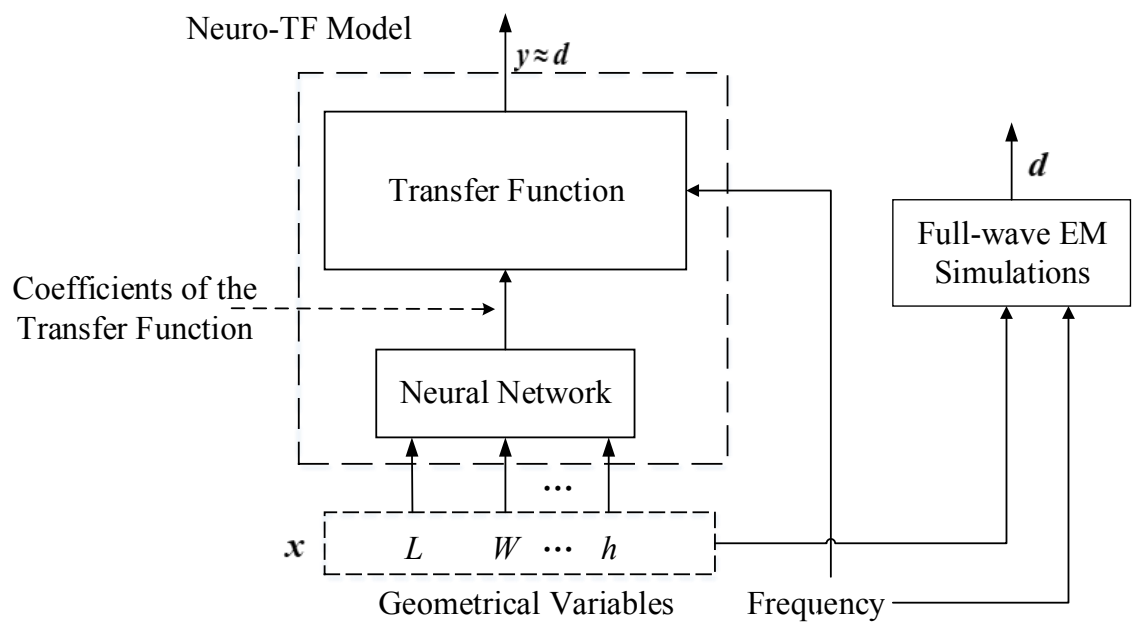

Figure 2.1: Structure of the rational function based neuro-TF model [17].

while the outputs of the model are EM responses (e.g., $S$-parameters) of microwave components. Let $\boldsymbol{x}$ represent the vector of geometrical variables. Let $\boldsymbol{y}$ represent the output vector of the model. Let $\boldsymbol{d}$ represent the desired outputs of the model. The development of a neuro-TF model consists of two stages. In the first stage, the relationship between the coefficients of the transfer function and geometrical parameters $\boldsymbol{x}$ are learnt by neural networks through a suitable training process. In the second stage, the overall model is further refined by adjusting the neural network weights such that the differences between $\boldsymbol{y}$ and $\boldsymbol{d}$ are minimized. 


\subsubsection{Parametric Modeling of Microwave Components Us- ing Combined Neural Network and Pole-Residue-Based Transfer Function}

To address the challenge of high sensitivities of the coefficients in the rational transfer functions for high-order problems, the transfer function has been expressed in the pole-residue form in [20]. The order of the transfer function may change as geometrical parameters change. To solve this order-changing problem, the authors

of [20] presented a pole-tracking technique where new groups of poles/residues are added to retain the consistency of the order of transfer function between different geometrical parameter values.

Figure 2.2 illustrates the structure of the pole-residue-based neuro-TF model [20]. The model consists of neural networks and pole-residue-based transfer functions. The model inputs contains a set of geometrical variables and frequency, while the model outputs are EM responses (e.g., $S$-parameters) of the microwave component. The development of the neuro-TF model mainly consists of three parts, i.e., a novel pole-residue tracking technique to solve the order-changing problem, preliminary training of neural networks, and pole-residue-based neuro-TF model refinement. In the first stage, several poles are chosen to be split into two overlapping poles, and then new groups of poles/residues are added to retain the consistency of the order of transfer function between different geometrical parameter values. In the second stage, the relationship between the poles/residues in the transfer function and geometrical parameters are learnt by neural networks by a proper training process. In the last stage, the overall model is further refined by performing a 


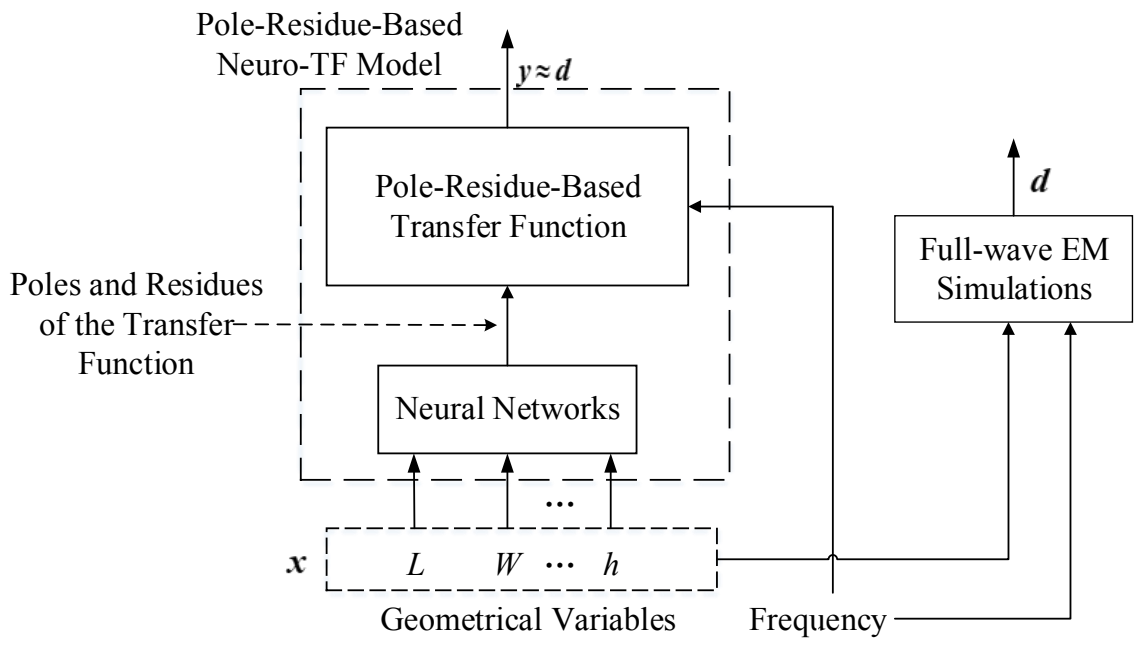

Figure 2.2: Structure of the pole-residue-based neuro-TF model, from [20]. $\boldsymbol{x}$ represents the vector of geometrical variables. $\boldsymbol{y}$ represents the output vector of the model. $\boldsymbol{d}$ represents the desired outputs of the model.

refinement training process.

\subsubsection{Parametric Modeling of Microwave Components Us- ing Adjoint Neural Networks and Pole-Residue Trans- fer Functions with EM Sensitivity Analysis}

As a further extension of [20], another sensitivity-analysis-based neuro-TF model has been proposed in [21], where EM sensitivity information has been exploited to achieve a faster model development process. The structure of the sensitivity-based neuro-TF model is shown in Fig. 2.3. The model consists of the original neuro-TF model and the adjoint neuro-TF model. The same inputs (i.e., geometrical variables and frequency) are shared between the original model and the adjoint model. The outputs of the adjoint model contain the derivatives of $\boldsymbol{y}$ (i.e., the original model 


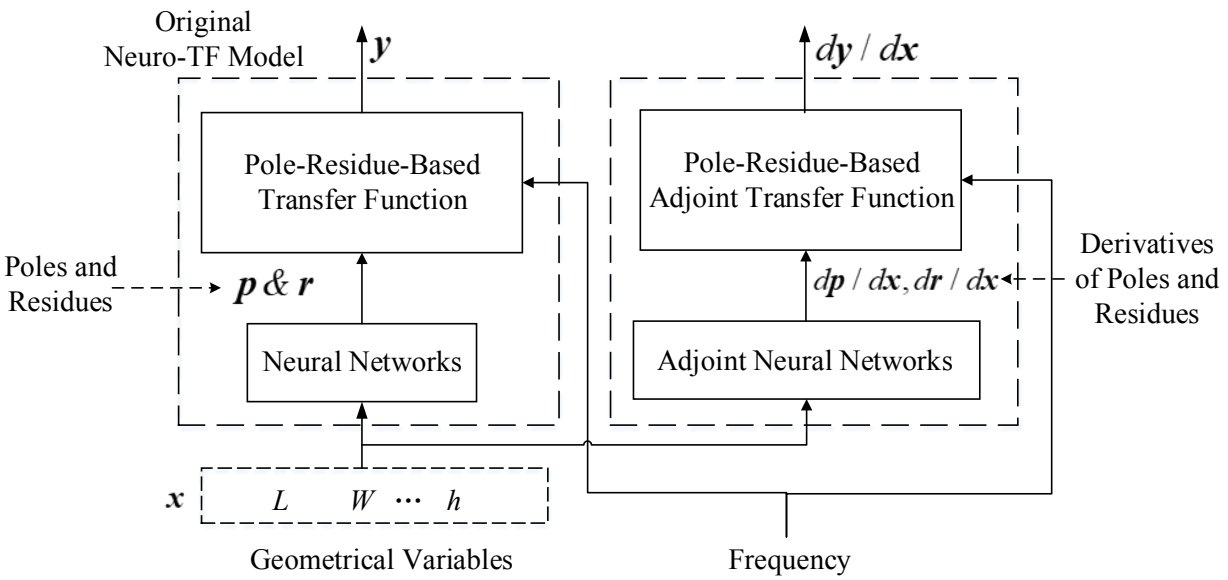

Figure 2.3: Structure of the sensitivity-analysis-based neuro-TF model [20]. $\boldsymbol{x}$ represents the vector of geometrical variables, $\boldsymbol{y}$ represents the output vector of the transfer function, and $d \boldsymbol{y} / d \boldsymbol{x}$ denotes the derivatives of $\boldsymbol{y}$ with respect to $\boldsymbol{x}$.

outputs) with respect to geometrical variables $\boldsymbol{x}$. Let $d \boldsymbol{y} / d \boldsymbol{x}$ denote the outputs of the adjoint model. Then, the outputs of the overall model contain both $\boldsymbol{y}$ and $d \boldsymbol{y} / d \boldsymbol{x}$.

The input-output EM behavior and the EM sensitivity information are generated from EM simulation simultaneously. Then, they are used to train the parametric model. The use of sensitivity information allows one to reduce the amount of training data to achieve desired model accuracy, accelerating the overall model development process. In addition, the sensitivity information also helps to solve the order-changing problem when the step sizes between geometrical parameter values are large. 


\subsubsection{Transfer Function Based Parametric Modeling Method Incorporating the Model Order Reduction Technique}

For all the aforementioned neuro-TF modeling methods, the initial training data of neural networks, i.e., the coefficients (or poles/residues) in transfer functions corresponding to a given set of EM responses, are obtained by the vector fitting technique [22]. Recently, another alternative transfer function based parametric modeling method that does not rely on the use of the vector fitting technique has been reported in [23] and [24]. The transfer functions used in this alternative method are derived from an application of the model-order reduction (MOR) technique. The poles/residues in the transfer function are obtained directly from full-wave EM simulations instead of through a vector fitting process. Thanks to the model order reduction (MOR) technique, the order of transfer function between different geometrical parameter values can be consistent. Therefore, one can use a constant order for the transfer function at different geometrical parameter values to avoid the order-changing problem in traditional neuro-TF modeling methods. The reason is that, unlike traditional neuro-TF modeling methods, the changes of the lessdominant poles are not random with respect to geometrical parameters for a fixedorder transfer function.

Uncertainties, introduced by the manufacturing process, pose inherent randomness on both geometrical dimensions and material properties of microwave components. Under this consideration, performing yield-driven optimization becomes an essential step in manufacturability-driven designs in a time-to-market development environment [25], [26]. The two decades after 1970 have witnessed the development 
of various yield optimization approaches, such as Monte Carlo-based approaches [25], [26], [80]-[82] and geometrical approaches [83]-[85]. These methods are developed mostly for circuit-based yield-driven design. Since the 1990s, electromagnetic (EM) simulations have been increasingly used in microwave design [35], [86]-[89]. However, compared with circuit-based yield-driven design, EM-based yield optimization is much more challenging. Simply replacing circuit simulations by EM simulations in conventional yield optimization approaches is not suitable because the requirement of a large number of EM simulations in yield optimization is computationally prohibitive. To alleviate the aforementioned difficulty, space mapping optimization method has been introduced to the yield-driven design of microwave structures. Space mapping employs computationally fast coarse models to reduce the evaluation cost of the computationally expensive EM fine models. The number of EM simulations required in yield optimization is expected to be reduced as all the EM simulations are attributed to calibrating the coarse model at each space mapping iteration. In the following section, we present a review of some initiative space mapping based methods for EM-based yield-driven design.

\subsection{Space Mapping-Based Yield-Driven EM Op- timization Methods \\ 2.3.1 Yield-Driven EM Optimization Using SM-Based Neu- ralmodels}

In [27], space mapping neuromodels have been used in an efficient EM-based yield optimization procedure. In this technique, an equivalent circuit model is taken as 
the coarse model, while full-wave EM simulation is taken as the fine model. The mapping function between the inputs (i.e., geometrical parameters) to the coarse model and the inputs to the fine model is implemented by a neural network, whose internal weights are to be optimized so that the the responses of the coarse model are close to those of the fine model. Once the training process is finished, the input-mapped coarse model provides fast and accurate predictions of the statistical properties for the EM responses, and can be utilized in subsequent yield estimation and optimization of microwave circuits. Nevertheless, this method does not update the surrogate during yield optimization. Instead, they require the surrogate to be initially constructed to be valid in a large neighborhood in the design parameter space. This means that, in the surrogate model development phase, a large number of EM simulations have to be performed, which is computationally expensive. The overall flow chart of the yield optimization procedure using SM-based neuralmodels is illustrated in Fig. 2.4.

\subsubsection{The Ellipsoidal Technique for Yield-Driven EM Opti- mization Using Space Mapping Surrogates}

In [28], space mapping has been combined with a modified ellipsoidal technique and then applied to yield optimization of microwave circuits. In this work, the SM surrogate is constructed by adding a linear mapping function to both inputs and outputs of the coarse model. The mapping parameters are updated in each SM iteration through a parameter extraction (PE) process. Both the EM responses at the nominal points in preceding iterations and the EM sensitivities at the nominal 


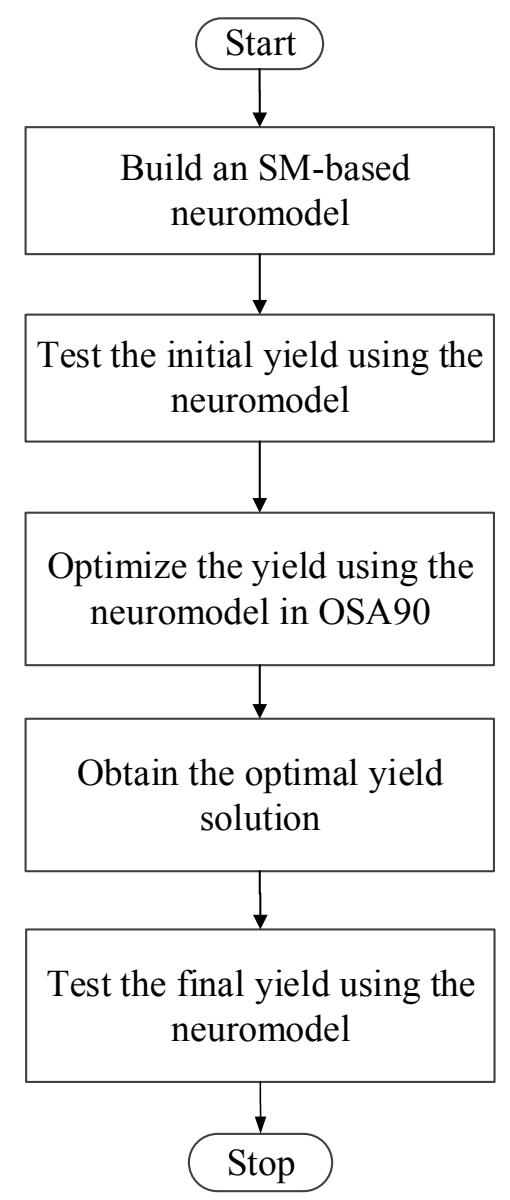

Figure 2.4: An illustration of the yield optimization procedure using SM-based neuralmodels.

point in current iteration are used to in the PE process enhance the accuracy of the surrogate model. Then, with the updated surrogate, a modified ellipsoidal technique is applied to find the optimal yield solution in each iteration. The SM surrogate provides a good approximation to the actual feasible region in the design parameter space with significantly reduced number of EM simulations, accelerating 


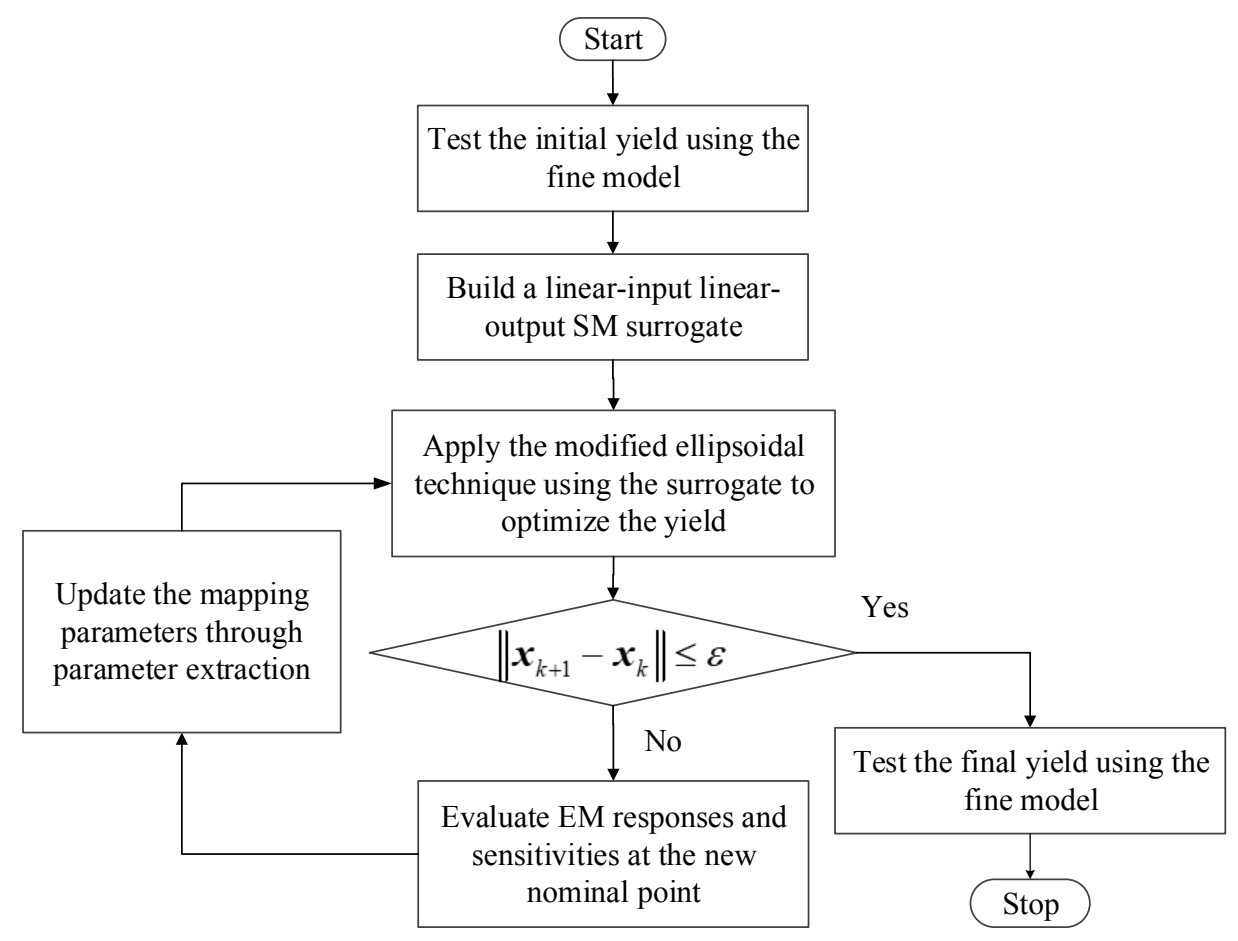

Figure 2.5: A summary of the yield optimization framework using the SM-based ellipsoidal technique.

the overall EM-based yield optimization process. The overall flow chart of the yield optimization procedure for this SM-based ellipsoidal technique is shown in Fig. 2.5.

\subsubsection{Yield-Driven EM Optimization Using Tuning Space Mapping Surrogates}

In [29], a tuning space-mapping surrogate based yield estimation and optimization technique has been presented. The responses of the surrogate are corrected to achieve enhanced yield estimation accuracy, facilitating the overall yield optimization process. Fig. 2.6 shows the flow chart of the overall yield optimization process. 


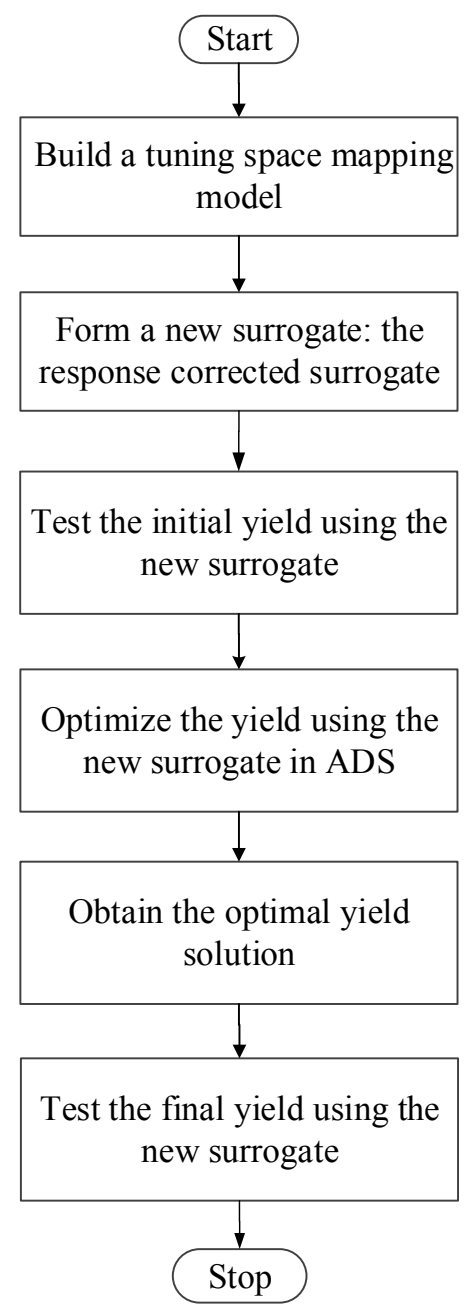

Figure 2.6: An illustration of the yield optimization procedure using tuning SM surrogates. 


\subsubsection{Yield-Driven EM Optimization Via a Non-Derivative Trust Region Approach and Space Mapping Surro- gates}

In [30], a non-derivative trust region approach (i.e., the new unconstrained optimization algorithm, NEWUOA) has been been combined with SM for optimizing the yield of microwave circuits. The generalized SM surrogate is initially constructed with an input and output linear space mapping, and then reconstructed during yield optimization by parameter extraction. Jacobian matrices of the EM responses are evaluated and used in the parameter extraction optimization process to enhance the accuracy of surrogate models. Moreover, to achieve an additional speedup for the yield estimation process, the Latin Hypercube Sampling (LHS) [90] is employed to generate the EM samples. An illustration of the overall yield optimization process using this technique is shown in Fig. 2.7.

\subsection{Feature-Based Yield-Driven EM Optimization Methods}

All the aforementioned space mapping-based approaches require the availability of an equivalent circuit coarse model. In many practical cases, equivalent circuit coarse models are not always available [91]. More recently, feature-based methods have been studied and applied to yield estimation and optimization of microwave structures [31], [32], [92]. In [31] and [92], a yield estimation technique exploiting feature-based statistical analysis has been presented. Yield optimization is then performed by optimizing the feature-based model using a pattern search algorithm. 


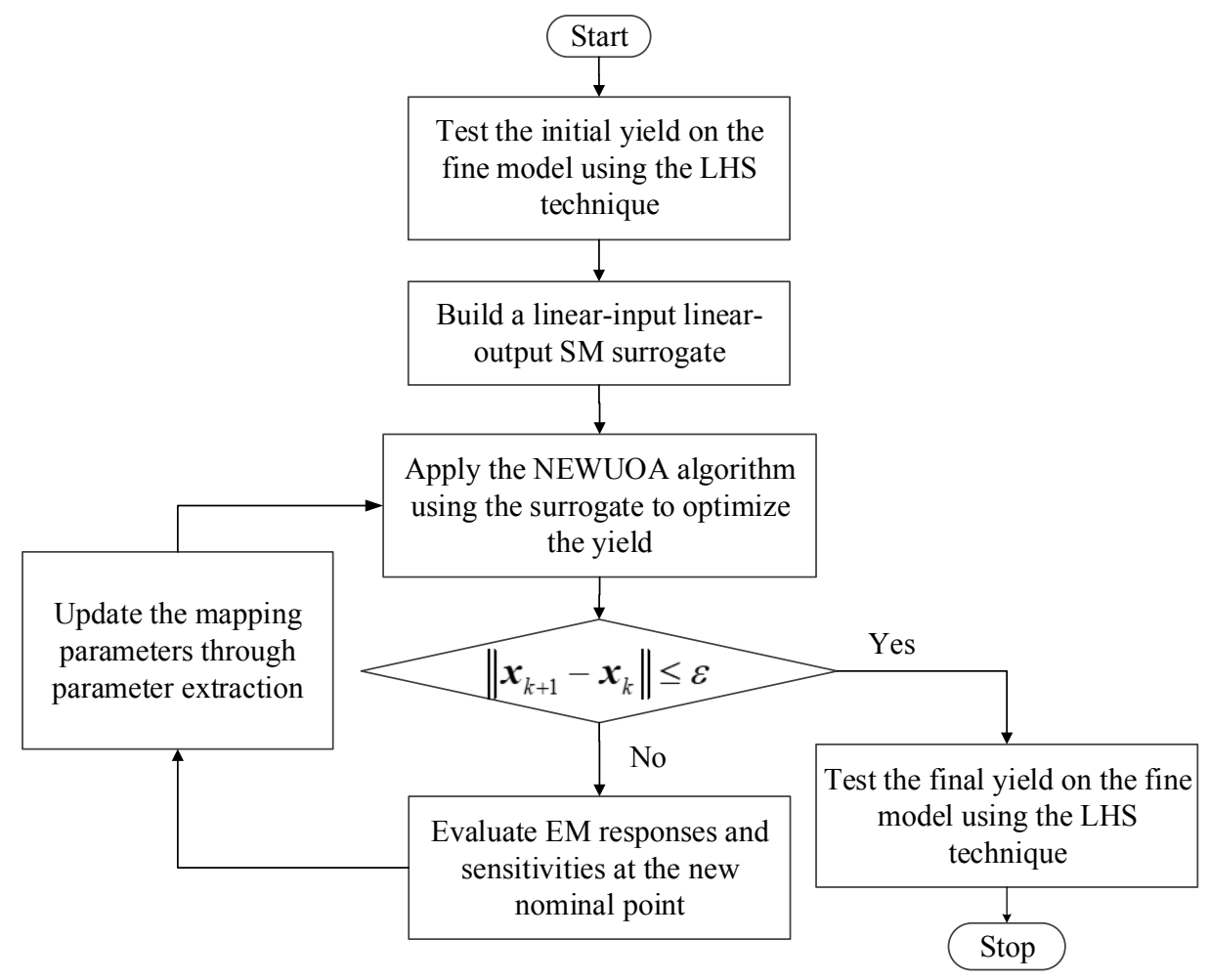

Figure 2.7: An illustration of the overall yield optimization process using the NEWUOA algorithm and SM surrogates.

In [32], a correction method for feature parameters has been described to allow yield estimation of microwave filters. Yield optimization is then formulated as a constrained optimization problem and solved accordingly. In this technique, a new set of feature parameters called specification frequencies $s$ are defined. Feature frequency parameters $\boldsymbol{f}$ and ripple height parameters $\boldsymbol{t}$ are also obtained. After generating the EM data at the nominal point as well as a number of geometrical samples using star distribution. The mappings from " $\boldsymbol{x}$-space" to the spaces of the feature frequency parameters $\boldsymbol{f}$ and specification frequencies $\boldsymbol{s}$ are constructed. 
With these mappings, the feature parameters can be predicted with respect to each change of design parameters. The mapping between $\boldsymbol{t}$ and $\boldsymbol{f}$ is determined in a similar way. Finally, the yield is estimated by checking whether the predicted feature parameters violate the specifications or not for all the random outcomes of $\boldsymbol{x}$. Yield optimization is then done by solving a constrained optimization problem. The overall yield optimization procedure is illustrated in Fig. 2.8, where $n$ represents the dimension of statistical variables.

\subsection{Polynomial Chaos Expansion Based Approach to Statistical Analysis and Yield Estimation of Microwave Circuits}

At each iteration of yield optimization, the prediction of yield values (also referred to as yield estimation) is typically involved. Recently, approaches based on polynomial chaos expansion (PCE) [36] have emerged as favorable alternatives for yield estimation and statistical analysis in the microwave area, such as [37]-[47]. In [37], for example, PCE has been used to expand the time-domain electric and magnetic field into orthogonal polynomial chaos basis functions of uncertain mesh parameters. In [38], a decoupled PCE and its applications to statistical analysis and yield estimation of high-speed interconnects have been reported. In [39], a non-intrusive formulation of the PCE approach has been applied to quantify the uncertainties in deterministic models of the indoor radio channel. It has been demonstrated that the PCE approach shows significant computational advantages over the traditional Monte Carlo analysis and can be regarded as a powerful tool in statistical analysis 


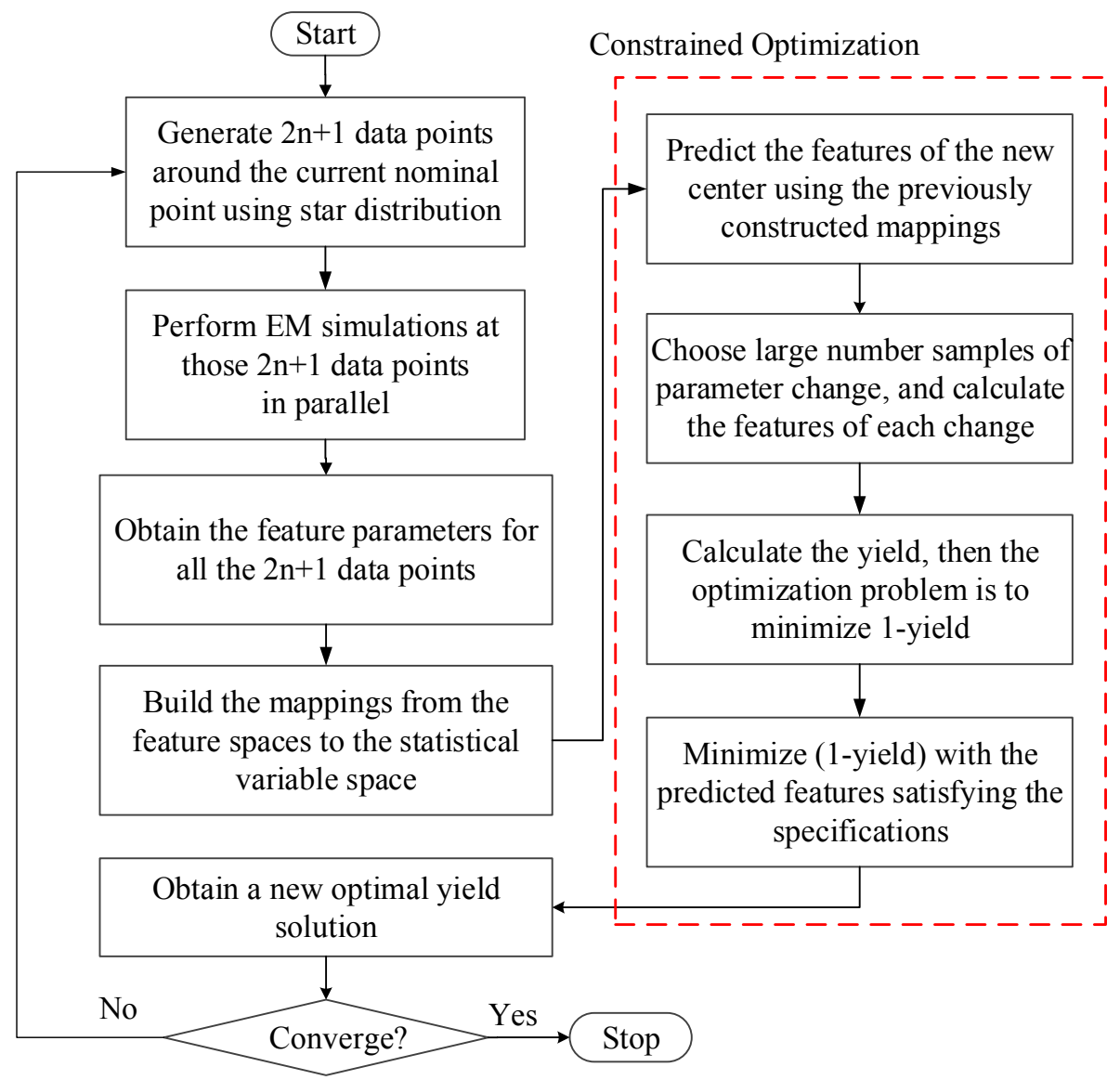

Figure 2.8: An illustration of the overall yield optimization process using the featurebased method presented in [32].

and yield estimation of microwave structures. Next, we present the formulation of the PCE approach and illustrate the idea of performing statistical analysis and yield estimation using PCE. 


\subsubsection{Formulation of Polynomial Chaos Expansion Approach}

A key component of PCE-based statistical analysis is performing a transformation of parameters from the original random parameters $\boldsymbol{x}$ to independent standard random parameters $\boldsymbol{\xi}$ then applying the stochastic expansion in the transformed space [93]. The transformation can be denoted as $\boldsymbol{\xi}=T\left(\boldsymbol{x}^{0}, \boldsymbol{x}\right)$. Let $R_{j}(\boldsymbol{\xi})$ be the EM response at the frequency of interest that corresponds to the $j$-th design specification sample. In $\mathrm{PCE}$, the functional form between $R_{j}(\boldsymbol{\xi})$ and $\boldsymbol{\xi}$ is approximated by the sum of weighted orthogonal basis polynomials in terms of the standard random parameters $\boldsymbol{\xi}$ as follows $[36]$

$$
R_{j}(\boldsymbol{\xi})=\sum_{i=0}^{P} a_{i j} \Phi_{i}(\boldsymbol{\xi}),
$$

where $\Phi_{i}(\cdot)$ is the generalized polynomial chaos basis function. The optimal bases to construct the multi-variate basis depend on the continuous probability distribution types of the design parameters. $a_{i j}$ is the weighting coefficient for the $i$-th basis function of the $j$-th EM response. $P+1$ is the number of terms in (2.1), given by

$P+1=\frac{(n+D) !}{n ! \cdot D !}$, where $n$ is the dimension of $\boldsymbol{x}$, and $D$ is the highest polynomial order in the expansion.

Depending on how the PCE coefficients are evaluated, PCE-based approaches can be classified into intrusive and non-intrusive approaches. The non-intrusive PCE approach evaluates the PCE coefficients $a_{i j}$ using the orthogonality condition of the polynomial chaos basis functions $\Phi_{i}(\boldsymbol{\xi})$. Specifically, the PCE coefficients $a_{i j}$ are found by

$$
a_{i j}=\frac{\int_{\Omega^{n}} R_{j}(\boldsymbol{\xi}) \Phi_{i}(\boldsymbol{\xi}) \rho(\boldsymbol{\xi}) d \boldsymbol{\xi}}{\int_{\Omega^{n}} \Phi_{i}^{2}(\boldsymbol{\xi}) \rho(\boldsymbol{\xi}) d \boldsymbol{\xi}},
$$


where $\Omega^{n}$ is the $n$-dimensional space of all possible values of $\boldsymbol{\xi} . \rho(\boldsymbol{\xi})$ is the joint probability density function of the standard random parameters $\boldsymbol{\xi}$. The multidimensional integration in (2.2) can be evaluated using numerical quadrature, e.g.,

$$
\begin{gathered}
\int_{\Omega^{n}} R_{j}(\boldsymbol{\xi}) \Phi_{i}(\boldsymbol{\xi}) \rho(\boldsymbol{\xi}) d \boldsymbol{\xi} \approx \sum_{l=1}^{M} R_{j}\left(\boldsymbol{\xi}^{(l)}\right) \Phi_{i}\left(\boldsymbol{\xi}^{(l)}\right) w^{(l)}, \\
\int_{\Omega^{n}} \Phi_{i}^{2}(\boldsymbol{\xi}) \rho(\boldsymbol{\xi}) d \boldsymbol{\xi} \\
\approx \sum_{l=1}^{M} \Phi_{i}^{2}\left(\boldsymbol{\xi}^{(l)}\right) w^{(l)}
\end{gathered}
$$

where $\boldsymbol{\xi}^{(l)}$ and $w^{(l)}$ are the integration quadrature points (also called "nodes") and weights in the " $\xi$-space", respectively. $M$ is the total number of integration quadra-

ture points. $R_{j}\left(\boldsymbol{\xi}^{(l)}\right)$ is the EM response evaluated at the $l$-th sampling point in the original random space. To reduce the computational costs for multi-dimensional numerical integration, sparse grid techniques are typically applied [94].

\subsubsection{Performing Statistical Analysis and Yield Estimation Using PCE Approach}

One valuable feature of the PCE approach is that, once the coefficients $a_{i j}$ are computed, the statistical properties of the stochastic quantity $R_{j}(\boldsymbol{\xi})$, e.g., mean $\mu_{j}$ and variance $\sigma_{j}^{2}$ can be obtained analytically through these coefficients in a simple closed form [39]

$$
\begin{gathered}
\mu_{j}=E\left(R_{j}\right)=a_{0 j}, \\
\sigma_{j}^{2}=E\left[\left(R_{j}-\mu_{j}\right)^{2}\right]=\sum_{i=1}^{P} a_{i j}^{2}\left(\sum_{l=1}^{M} \Phi_{i}^{2}\left(\boldsymbol{\xi}^{(l)}\right) w^{(l)}\right) .
\end{gathered}
$$

To perform yield estimation of a given EM structure, a number of PCE models have to be constructed at all the frequencies of interest according to (2.1)-(2.4). More 
specifically, a single PCE model has to be constructed for each frequency point under consideration. In other words, the total number of PCE models is equal to the total number of frequency points in the whole frequency range of interest. Then, a Monte Carlo analysis needs to be performed on the PCE models taking into consideration all the design specifications [95].

\subsection{Conclusion}

In this chapter, we have provided a literature review of popularly used parametric modeling and yield-driven optimization methods of microwave passive components. We first present an overview of the ANN-based parametric modeling method in the past years. Both the knowledge-based neural network models and the neuro-TF modeling methods have been reviewed. Then, a number of existing yield-driven EM optimization methods have been briefly illustrated. We start with a group of SM-based methods, namely, the neural SM based method, the tuning SM-based method, the method combining SM with the ellipsoidal technique, and the method combining SM with a non-derivative trust region approach. Then, we review two recent feature-based yield-driven EM optimization methods. Lastly, a review of the PCE approaches to statistical analysis and yield estimation of microwave circuits has been provided. In the next chapter, a novel training approach for parametric modeling of microwave passive components using Padé via Lanczos and EM sensitivities is proposed. 


\section{Chapter 3}

\section{Parametric Modeling of Microwave Passive Components Using PVL and EM Sensitivities}

\subsection{Introduction}

In this chapter, we propose a novel training approach for parametric modeling of microwave passive components that can address large geometrical variations. In the proposed approach, the EM responses (e.g., $S$-parameters) of passive components versus frequency are represented by pole-zero-gain transfer functions derived from an application of the finite element method and the matrix Padé via Lanczos algorithm. To address the issue of mismatch of poles/zeros when the geometrical variations are large, we propose a novel sensitivity-analysis-based pole/zero-matching algorithm to obtain the correct correspondences between the poles/zeros at one geometrical parameter value and the poles/zeros at the other geometrical parameter value. The proposed algorithm exploits the EM sensitivity information, which provides useful knowledge for the direction of movement of the poles/zeros, to predict the new 
positions of the poles/zeros for each change of geometrical parameters in the multidimensional parameter space. The predicted new positions are then used to guide the matching process of poles/zeros between different geometrical parameter values. The use of the EM sensitivities allows the proposed algorithm to obtain more reliable pole/zero-matching solutions than existing methods when the geometrical variations are large. Using the matched poles/zeros to train the neural networks allows us to have fast and reliable predictions for the poles/zeros subject to large geometrical variations, consequently increasing the accuracy and robustness of the overall model. Compared with the existing methods, the proposed approach can obtain better accuracy in challenging applications involving large geometrical variations. Once developed, the model can provide fast and accurate prediction of the EM behavior of microwave components with geometrical parameters as variables, and can be further utilized in high-level microwave circuit and system designs.

\subsection{Proposed Training Approach for Parametric Modeling of Microwave Passive Components}

The structure of the proposed neuro-TF model is shown in Fig. 3.1. The model consists of two main components, i.e., neural networks and transfer functions in the complex propagation space. The outputs of the model are the EM responses (e.g., $S$-parameters) of the microwave component, while the inputs contain geometrical variables of the EM structure and frequency. Let $\boldsymbol{x}$ and $\boldsymbol{y}$ be the vector of geometrical variables and the output vector of the proposed neuro-TF model, respectively. Let $\boldsymbol{d}$ be the desired output vector of the model. Let $\boldsymbol{w}$ denote the neural network 


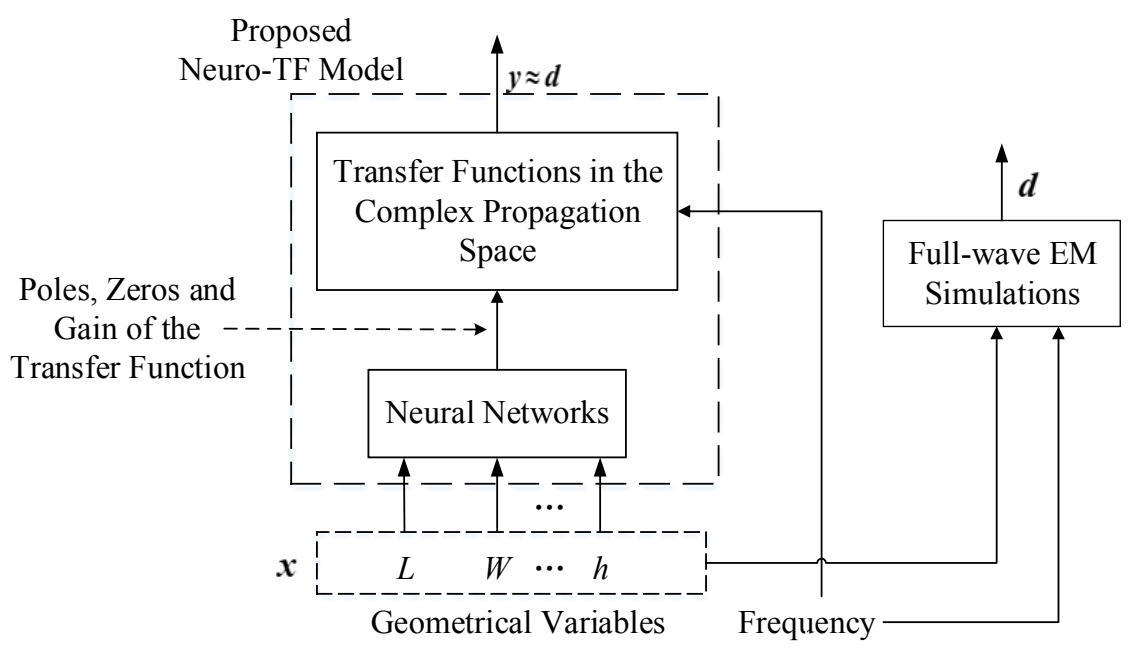

Figure 3.1: Structure of the proposed neuro-TF model. $\boldsymbol{x}$ represents the geometrical variables. $\boldsymbol{y}$ represents the outputs (e.g., $S$-parameters) of the transfer function in the complex propagation space. $\boldsymbol{d}$ represents the outputs of EM simulations.

weights. The purpose of training the model is to minimize the differences between $\boldsymbol{y}$ and $\boldsymbol{d}$ at different values of $\boldsymbol{x}$ by adjusting $\boldsymbol{w}$ using a certain optimization algorithm.

Assume that the finite element method (FEM) is applied to solve the EM wave propagation problems in the frequency domain [96], and that the MPVL algorithm is applied to the FEM system for fast frequency sweep [97]. The MPVL algorithm uses an iterative process to reduce the original system matrix in FEM to a $q \times q$ tridiagonal matrix $\boldsymbol{T}$, where $q$ is the order of the reduced-order models (ROMs) [97]. The outcomes from MPVL also include a $q \times 1$ matrix $\boldsymbol{\rho}$ and a $q \times N_{\text {in }}$ matrix $\boldsymbol{\eta}$, where $N_{\text {in }}$ is the number of unknowns on the input port. Note that $\boldsymbol{T}, \boldsymbol{\rho}$, and $\boldsymbol{\eta}$ are all in general complex-valued matrices, and their elements all change as geometrical parameters change.

Let $H(\omega)$ represent the frequency response (e.g., $\left.S_{11}\right)$ of microwave passive com- 
ponents, where $\omega$ is the angular frequency. Let $\gamma(\omega)=j \beta(\omega)$, where $\beta(\omega)$ is the propagation constant. Given $\boldsymbol{T}, \boldsymbol{\rho}$, and $\boldsymbol{\eta}$, the transfer function $H(\omega)$ can be written in the following pole-zero-gain form [97] (see Appendix for detailed information):

$$
H(\omega)=\gamma(\omega) K \frac{\prod_{i=1}^{q-1}\left(\gamma(\omega)-\gamma_{0}-z_{i}\right)}{\prod_{i=1}^{q}\left(\gamma(\omega)-\gamma_{0}-p_{i}\right)}+c,
$$

where $p_{i}$ represents the poles, $z_{i}$ the zeros, $K$ the gain of the transfer function, and $c$ a constant. $q$ is the order of the ROMs and is also the number of poles in the transfer function. $\gamma_{0}=\gamma\left(\omega_{0}\right)$ and $\omega_{0}$ is the expansion point in frequency. To obtain the poles, zeros, and gain in $H(\omega)$, the FEM, the MPVL algorithm, and an eigendecomposition process have to be executed, as given by Appendix. Note that the poles and zeros defined in (3.1) are not in the well-known "s-space" $(s=j \omega)$. We refer to the poles/zeros in (3.1) as the poles/zeros in the complex propagation space, or the " $\gamma$-space" in this chapter.

As geometrical parameters change, the poles and zeros, as well as the gain change accordingly. Further, as geometrical parameters change to a new value, the FEM, the MPVL algorithm, and the eigendecomposition process have to be re-executed to obtain a new set of poles, zeros and gain. However, two issues arise as one recomputes the new set of poles, zeros, and gain. Firstly, the time-consuming nature of the FEM makes the evaluation of the poles/zeros/gain computationally expensive, and repetitive changes of the geometrical parameter values necessitate massive evaluations of the poles/zeros/gain. To make these evaluations fast and accurate, we propose to use neural networks to learn the relationship between the poles/zeros/gain and geometrical parameters by a proper training process. Sec- 
ondly, as the poles/zeros are recomputed by MPVL and eigendecomposition each time we change the geometrical parameters, the indices of the poles/zeros after the recomputation may not have clear correspondences with those before the recomputation. In other words, without sorting, the sequence of the unsorted poles/zeros at one geometrical parameter value may not correspond with that at the other geometrical parameter value. Consequently, the poles/zeros at one geometrical value do not match with those at the other geometrical value. This may lead to wrong predictions of the poles/zeros for a new change of geometrical parameters. We call this issue the issue of mismatch of poles/zeros in this chapter. In the following sections, we describe this issue in more detail and propose a novel sensitivity-analysis-based algorithm to solve it.

\subsubsection{The Issue of Mismatch of Poles and Zeros}

We first use a simple fictitious example with two real-valued poles $\left(p_{1}\right.$ and $\left.p_{2}\right)$ and one geometrical variable $(L)$ to illustrate the issue of mismatch of poles. As $L$ changes, the values of $p_{1}$ and $p_{2}$ have to be recomputed. Table 3.1 shows the original unsorted poles, where the indices of the poles at one geometrical parameter value do not correctly correspond to those at the other geometrical parameter value. A discontinuity is observed for both poles as the geometrical variable changes. If we use these original unsorted poles to interpolate the values of the poles at new geometrical parameter values, the interpolation results may be totally wrong. For example, using the information in Table 3.1, the interpolation results at a new value of $L(12.05 \mathrm{~mm})$ are $p_{1}=14.195$ and $p_{2}=14.2$. In reality, both of these 
Table 3.1: The Original Unsorted Poles Versus Changing Values of the Geometrical Parameter $L$ - An Illustrative Example

\begin{tabular}{c|c|c}
\hline \hline$L(\mathrm{~mm})$ & $p_{1}$ & $p_{2}$ \\
\hline 12.02 & 3.68 & 24.61 \\
\hline 12.04 & 3.72 & 24.64 \\
\hline 12.06 & 24.67 & 3.76 \\
\hline 12.08 & 24.70 & 3.80 \\
\hline 12.10 & 24.73 & 3.84 \\
\hline \hline
\end{tabular}

Table 3.2: The Poles of Table 3.1 After Being Sorted Correctly

\begin{tabular}{c|c|c}
\hline \hline$L(\mathrm{~mm})$ & $p_{1}$ & $p_{2}$ \\
\hline 12.02 & 3.68 & 24.61 \\
\hline 12.04 & 3.72 & 24.64 \\
\hline 12.06 & 3.76 & 24.67 \\
\hline 12.08 & 3.80 & 24.70 \\
\hline 12.10 & 3.84 & 24.73 \\
\hline \hline
\end{tabular}

two poles from interpolation are wrong. Table 3.2 shows the poles after we sort the original poles in Table 3.1 correctly. If we use these sorted poles to interpolate the values of $p_{1}$ and $p_{2}$ at $L=12.05(\mathrm{~mm})$, the correct solutions $p_{1}=3.74$ and $p_{2}=24.655$ can be obtained. This example shows that it is necessary to sort the poles at each geometrical parameter value into the correct sequence in order to have correct interpolation results for the poles at new geometrical parameter values.

A simple solution to this issue is to sort the poles/zeros according to their magnitudes at each geometrical parameter value, as shown in Table 3.2. Such sorting is usually available in many numerical computing tools such as MATLAB. However, 
sorting the poles simply according to their magnitudes cannot solve the issue of mismatch of poles completely. To illustrate, Fig. 3.2 shows another example where the poles are obtained from a fifth-order waveguide bandpass filter. The two poles are recomputed as the geometrical variable $L$ changes to any new values in the range of $14.01(\mathrm{~mm})$ to $14.95(\mathrm{~mm})$, where $L$ is the distance from one pair of irises to the waveguide wall. The original unsorted poles in Fig. 3.2(a) are directly obtained from eigendecomposition in a typical numerical computing tool (i.e., MATLAB). Using the unsorted poles in Fig. 3.2(a), interpolation results for the poles at new geometrical parameter values may be wrong. Consequently, the overall model developed using these original poles will be inaccurate and non-robust. Fig. 3.2(b) shows the poles after sorting the original ones according to their magnitudes. It is seen that the issue of mismatch of poles still exists after the sorting. This demonstrates that simply sorting the poles according to their magnitudes cannot solve the issue completely.

Fig. 3.2(c) shows the correct sorting results of the two poles. In comparison with those in Fig. 3.2(a) and Fig. 3.2(b), the changes of the poles in Fig. 3.2(c) are more continuous and smooth. Therefore, to allow reliable interpolations for the poles/zeros, the original poles/zeros need to be sorted/matched correctly before being used as training data for the neural networks in the proposed model. Specifically, the basic problem we are considering can be stated as follows: Given two sets of poles corresponding to two different geometrical parameter values, we need to determine which pole in one set corresponds to a given pole in the other set. We refer to the process to determine such correspondences as the pole-matching process 


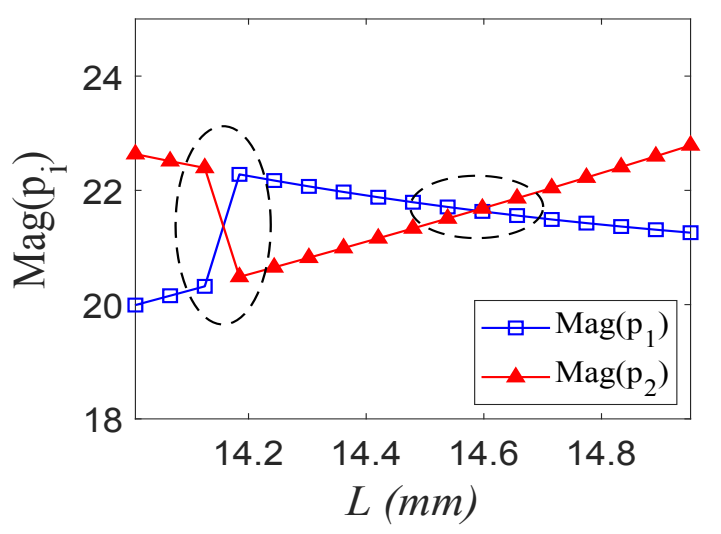

(a)

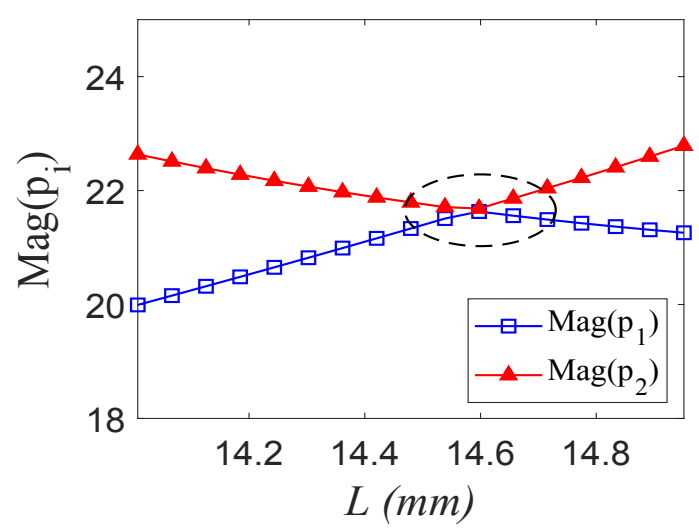

(b)

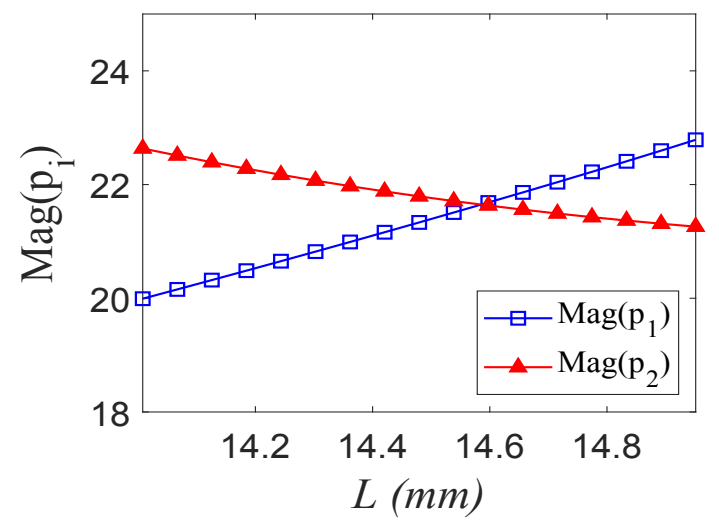

(c)

Figure 3.2: Illustration of the mismatch issue of poles w.r.t. one geometrical variable using a fifth-order waveguide bandpass filter example: (a) The original unsorted poles obtained directly from the eigendecomposition processes; (b) The poles sorted according to their magnitudes; (c) Correct sorting results of the two poles. In both (a) and (b), the dashed circles indicate the areas where the issue of mismatch of poles occurs. 
in this chapter.

In practice, the original poles/zeros obtained from eigendecomposition are usually unsorted. Therefore, the indices for the poles/zeros at a geometrical parameter value may not be consistent with those at another geometrical parameter value. The two examples above show relatively easy cases where two poles are mismatched as one geometrical parameter changes. In many practical cases, as multiple geometrical parameters change, the poles/zeros move in a multi-dimensional space and multiple poles/zeros are simultaneously mismatched. Therefore, the mismatch pattern of the poles/zeros is much more complex and the correct solution of matching the poles/zeros turns out to be neither simple nor obvious.

Some research activities have been carried out to approach this problem recently, i.e., the distance-based pole-matching method presented in [23] and the continuation method presented in [24]. Both methods perform well for applications with small/medium geometrical variations. In this work, we focus on more challenging applications involving large geometrical variations. Accurate parametric models valid in a large region in the geometrical parameter space are useful for accelerating nominal and yield-driven design of microwave passive components. In the following section, we propose a novel sensitivity-analysis-based pole/zero-matching algorithm to address the issue of mismatch of poles/zeros. The proposed algorithm exploits the EM sensitivities of poles/zeros at a geometrical parameter value to predict the new positions of the poles/zeros at another geometrical parameter value in multidimensional parameter spaces. Then, it matches the poles/zeros such that the differences between the predicted poles/zeros and the original ones are minimal. The 
use of the EM sensitivities allows the proposed algorithm to obtain more reliable pole/zero-matching solutions than existing methods when the geometrical variations are large. This advantage ultimately makes the overall model developed using the proposed approach more accurate and robust than those developed by existing methods, especially for challenging applications with large geometrical variations.

\subsubsection{Sensitivity-Analysis-Based Pole/Zero-Matching Algo- rithm}

Let $\boldsymbol{x}$ be a vector containing all the geometrical parameters of the microwave structure, i.e., $\boldsymbol{x}=\left[x_{1}, x_{2}, \ldots, x_{m}\right]^{T}$, where $m$ is the dimension of $\boldsymbol{x}$. The proposed training approach for developing a neuro-TF model begins with the generation of training data (for both the neural networks and the overall model) by performing full-wave EM simulations at a set of geometrical parameter values. To do this, we change the value of $\boldsymbol{x}$ multiple times to obtain a set of samples of $\boldsymbol{x}$ (also called a set of geometrical parameter values in this chapter). Let $N$ represent the number of changes in geometrical parameter values. Let $X$ be the set containing all the geomet-

rical parameter values in the training data, i.e., $X=\left\{\boldsymbol{x}^{(0)}, \boldsymbol{x}^{(1)}, \ldots, \boldsymbol{x}^{(N)}\right\} . \boldsymbol{x}^{(0)}$ is the initial geometrical parameter value before any change, while $\boldsymbol{x}^{(1)}, \boldsymbol{x}^{(2)}, \ldots, \boldsymbol{x}^{(N)}$ are samples of $\boldsymbol{x}$ after $\boldsymbol{x}$ is changed following a certain sampling method. In general, $\boldsymbol{x}^{(n)}$ is the $n$th sample of $\boldsymbol{x}$, where $n=0,1, \ldots, N$. Let $T_{r}$ represent the index set of the training samples at different geometrical parameter values, i.e., $T_{r}=\{0,1, \ldots, N\}$. The total number of geometrical parameter values (samples) in $X$ is $N+1$. 
Let $\boldsymbol{p} \in \mathbb{C}^{q \times 1}$ and $\boldsymbol{z} \in \mathbb{C}^{(q-1) \times 1}$ be two vectors containing all the poles and zeros in $H(\omega)$ at a given geometrical parameter value, respectively. Then, we have $\boldsymbol{p}=\left[\begin{array}{lllll}p_{1} & p_{2} & \ldots & p_{q}\end{array}\right]^{T}$ and $\boldsymbol{z}=\left[\begin{array}{lllll}z_{1} & z_{2} & \ldots & z_{q-1}\end{array}\right]^{T}$, where $q$ and $q-1$ represent the number of poles and the number of zeros in $H(\omega)$, respectively. For each geometrical parameter value $\boldsymbol{x}^{(n)}, n \in T_{r}$, we apply the FEM to solve the EM propagation problem [96], reduce the order of the system resulting from FEM using the MPVL algorithm [97], and execute an eigendecomposition process (refer to Appendix for more details) to obtain the following three sets of data: $\left\{\boldsymbol{x}^{(n)}, \boldsymbol{p}^{(n)}\right\},\left\{\boldsymbol{x}^{(n)}, \boldsymbol{z}^{(n)}\right\}$, and $\left\{\boldsymbol{x}^{(n)}, K^{(n)}\right\} . K^{(n)}$ is the gain evaluated at $\boldsymbol{x}^{(n)} \cdot \boldsymbol{p}^{(n)}$ and $\boldsymbol{z}^{(n)}$ are two vectors containing the poles and the zeros evaluated at $\boldsymbol{x}^{(n)}$, respectively, i.e.,

$$
\begin{aligned}
& \boldsymbol{p}^{(n)}=\left[\begin{array}{llll}
p_{1}^{(n)} & p_{2}^{(n)} & \ldots & p_{q}^{(n)}
\end{array}\right]^{T}, \\
& \boldsymbol{z}^{(n)}=\left[\begin{array}{llll}
z_{1}^{(n)} & z_{2}^{(n)} & \ldots & z_{q-1}^{(n)}
\end{array}\right]^{T} .
\end{aligned}
$$

Assuming that sensitivity analysis is available from full-wave EM simulations, then we can obtain the matrices of EM sensitivities of poles and zeros w.r.t. geometrical variables at all the geometrical parameter values in $X$. For the $n$th geometrical parameter value $\boldsymbol{x}^{(n)}$, we apply the FEM and the MPVL algorithm to solve the EM wave propagation problem in the frequency domain, and perform EM sensitivity analysis to obtain the following two Jacobian matrices:

$$
\begin{aligned}
& \boldsymbol{J}_{p}^{(n)}=\left[\frac{\partial p_{1}}{\partial \boldsymbol{x}^{(n)}} \frac{\partial p_{2}}{\partial \boldsymbol{x}^{(n)}} \cdots \frac{\partial p_{q}}{\partial \boldsymbol{x}^{(n)}}\right]^{T}, \\
& \boldsymbol{J}_{z}^{(n)}=\left[\frac{\partial z_{1}}{\partial \boldsymbol{x}^{(n)}} \frac{\partial z_{2}}{\partial \boldsymbol{x}^{(n)}} \cdots \frac{\partial z_{q-1}}{\partial \boldsymbol{x}^{(n)}}\right]^{T},
\end{aligned}
$$


where $\boldsymbol{J}_{p}^{(n)} \in \mathbb{C}^{m \times q}, \boldsymbol{J}_{z}^{(n)} \in \mathbb{C}^{m \times(q-1)}, \frac{\partial p_{r}}{\partial \boldsymbol{x}^{(n)}}=\left.\frac{\partial p_{r}}{\partial \boldsymbol{x}}\right|_{\boldsymbol{x}=\boldsymbol{x}^{(n)}}(r=1, \ldots, q)$, and $\frac{\partial z_{r}}{\partial \boldsymbol{x}^{(n)}}=$ $\left.\frac{\partial z_{r}}{\partial \boldsymbol{x}}\right|_{\boldsymbol{x}=\boldsymbol{x}^{(n)}}(r=1, \ldots, q-1) . \boldsymbol{J}_{p}^{(n)}$ and $\boldsymbol{J}_{z}^{(n)}$ contain the EM sensitivities of poles and the EM sensitivities of zeros w.r.t. geometrical variables at $\boldsymbol{x}^{(n)}$, respectively. The sensitivities in $\boldsymbol{J}_{p}^{(n)}$ and $\boldsymbol{J}_{z}^{(n)}$ are both evaluated at $\boldsymbol{x}^{(n)}$. These sensitivity information are to be utilized in the subsequent pole/zero-matching process.

\subsubsection{Matching the Poles Between Two Neighboring Geometrical Pa- rameter Values}

Suppose that two neighboring geometrical parameter values are chosen from $X$ for the purpose of pole-matching. Let $\boldsymbol{x}^{(k)}$ and $\boldsymbol{x}^{(l)}\left(k \in T_{r}, l \in T_{r}\right)$ denote these two values. As geometrical parameters change from $\boldsymbol{x}^{(k)}$ to $\boldsymbol{x}^{(l)}$, all the poles at $\boldsymbol{x}^{(k)}$ move to new positions. To match the poles at $\boldsymbol{x}^{(l)}$ with those at $\boldsymbol{x}^{(k)}$, we first exploit the sensitivity information of the poles w.r.t. geometrical variables at $\boldsymbol{x}^{(k)}$ to predict the new positions of the poles at $\boldsymbol{x}^{(l)}$. Then, We compare the predicted pattern of the $q$ poles with the original unsorted pattern of the $q$ poles at $\boldsymbol{x}^{(l)}$. The purpose is to sort (and re-sort) the original pattern until the sorted pattern matches the predicted pattern. In other words, the $q$ poles at $\boldsymbol{x}^{(l)}$ are matched with those at $\boldsymbol{x}^{(k)}$ such that the overall differences between the actual and the predicted new positions of the poles at $\boldsymbol{x}^{(l)}$ are minimal. Let $\overline{\boldsymbol{p}}^{(l)}$ represent the predicted new positions of the poles at $\boldsymbol{x}^{(l)}$. By taking advantage of the EM sensitivities of the poles at $\boldsymbol{x}^{(k)}, \overline{\boldsymbol{p}}^{(l)}$ is obtained as follows:

$$
\overline{\boldsymbol{p}}^{(l)}=\boldsymbol{p}^{(k)}+\boldsymbol{J}_{p}^{(k)} \Delta \boldsymbol{x},
$$


where $\Delta \boldsymbol{x}=\boldsymbol{x}^{(l)}-\boldsymbol{x}^{(k)}$, which denotes the change of geometrical parameters between $\boldsymbol{x}^{(l)}$ and $\boldsymbol{x}^{(k)} . \boldsymbol{J}_{p}^{(k)}$ represents the EM sensitivities of the poles at $\boldsymbol{x}^{(k)}$. Note that in Eqn. (3.6), a linear function is utilized to represent the relationship between the change of the poles and that of the geometrical parameters for two neighboring geometrical samples. However, in general, when geometrical parameters change inside the entire region covered by all the samples in the design parameter space, the change of the poles is typically a nonlinear function of the change of geometrical parameters.

Ideally, one should consider all the possibilities of matching the poles between $\boldsymbol{x}^{(k)}$ and $\boldsymbol{x}^{(l)}$ (i.e., all the sorting possibilities of sorting the poles at $\boldsymbol{x}^{(l)}$ ), and find the optimal pole-matching solution by comparing all of them. One possible candidate as a pole-matching solution is actually a permutation of the indices of the $q$ poles at $\boldsymbol{x}^{(l)}$. By permutating the indices of the poles, the optimal correspondence between the two sets of poles (one set at $\boldsymbol{x}^{(k)}$ and the other set at $\boldsymbol{x}^{(l)}$ ) can be obtained. Since the total number of permutations of $q$ indices is $q$ !, the total number of possible candidates as the pole-matching solution is $q$ ! as well. Thus, the search space for the optimal pole-matching solution would become extremely large if all the possible candidates are considered (e.g., when $q=12, q !=479001600$ ). To avoid the high computational complexity, we propose an efficient and effective method to seek suboptimal pole-matching solutions. With this method, the computational complexity of the proposed pole-matching algorithm can be reduced from $O(q !)$ to $O\left(q^{2}\right)$.

Let $\boldsymbol{D} \in \mathbb{R}^{q \times q}$ be a matrix containing the distances between the actual and the predicted new positions of the poles at $\boldsymbol{x}^{(l)}$. The elements in $\boldsymbol{D}$ are obtained as 
follows:

$$
\boldsymbol{D}_{i j}=\left\|\bar{p}_{i}^{(l)}-p_{j}^{(l)}\right\|
$$

where $i, j=1,2, \ldots, q \cdot \bar{p}_{i}^{(l)}$ represents the predicted new position for the $i$ th pole at $\boldsymbol{x}^{(l)}$, while $p_{j}^{(l)}$ represents the actual new position for the $j$ th pole at $\boldsymbol{x}^{(l)}$.

Given the matrix $\boldsymbol{D}$, the poles between $\boldsymbol{x}^{(k)}$ and $\boldsymbol{x}^{(l)}$ are matched in $q$ rounds. Let $r$ be a counter, where $r=1,2, \ldots, q$. In the $r$ th round, we find the index of the minimum element in $\boldsymbol{D}$, i.e.,

$$
\left(i_{r}, j_{r}\right)=\arg \min _{\substack{i \in\{1, \ldots, q\}, i \notin \Omega_{1}^{r} \\ j \in\{1, \ldots, q\}, j \notin \Omega_{2}^{r}}}\left\{\boldsymbol{D}_{i j}\right\},
$$

where $i_{r}$ and $j_{r}$ denote the row index and column index of the minimum element in $\boldsymbol{D}$, respectively. $\Omega_{1}^{r}$ is a set containing all the row indices of the minimum elements found in the rounds prior to round $r$, while $\Omega_{2}^{r}$ is a set containing all the column indices of the minimum elements found in the rounds prior to round $r . \Omega_{1}^{r}$ and $\Omega_{2}^{r}$ are both initialized as an empty set in the first round $(r=1)$ and updated in subsequent rounds as follows:

$$
\begin{aligned}
& \Omega_{1}^{r+1}=\Omega_{1}^{r} \cup\left\{i_{r}\right\}, \\
& \Omega_{2}^{r+1}=\Omega_{2}^{r} \cup\left\{j_{r}\right\},
\end{aligned}
$$

where $r=1, \ldots, q-1$. A small value of $\boldsymbol{D}_{i j}$ implies that the predicted new position of the $i$ th pole is close to the actual new position of the $j$ th pole. Hence, the $i$ th pole is very likely to move to the actual new position of the $j$ th pole at $\boldsymbol{x}^{(l)}$. By using this idea, we match the $i_{r}$ th pole at $\boldsymbol{x}^{(k)}$ with the $j_{r}$ th pole at $\boldsymbol{x}^{(l)}$ in round $r$. 
The above procedure terminates after $q$ rounds when all the poles of $\boldsymbol{x}^{(l)}$ have a one-to-one match with those of $\boldsymbol{x}^{(k)}$. Let $\Phi$ be a set containing the final polematching solution. Specifically,

$$
\Phi=\left\{\left(i_{r}, j_{r}\right) \mid r=1,2, \ldots, q\right\},
$$

where the $r$ th element in $\Phi$, i.e., $\left(i_{r}, j_{r}\right)$, implies that the $i_{r}$ th pole at $\boldsymbol{x}^{(k)}$ is matched with the $j_{r}$ th pole at $\boldsymbol{x}^{(l)}$.

Lastly, we sort the poles at $\boldsymbol{x}^{(l)}$ such that the original $j_{r}$ th pole is sorted into the new $r$ th pole, where $r=1, \ldots, q$. For example, if $q=3$ and $\Phi=\{(1,3),(2,1),(3,2)\}$, then the poles after the sorting are $\boldsymbol{p}^{(l)}=\left[p_{3}^{(l)} p_{1}^{(l)} p_{2}^{(l)}\right]^{T}$. By performing such sorting, each pole at $\boldsymbol{x}^{(l)}$ will have a correct match with that at $\boldsymbol{x}^{(k)}$. The flow chart of matching the poles between two neighboring geometrical parameter values is shown in Fig. 3.3.

\subsubsection{Enumerating All the Geometrical Parameter Values for Pole- Matching}

The pole-matching algorithm described above is to match the poles between any two neighboring geometrical parameter values. i.e., between $\boldsymbol{x}^{(k)}$ and $\boldsymbol{x}^{(l)}$. In reality, many geometrical parameter values (samples) exist. Two concerns have to be dealt with while matching the poles at all the geometrical samples in $X$. Firstly, the matching of the poles at all the geometrical samples should follow a common set of reference indices of the poles. Secondly, to avoid massive number of EM simulations especially in applications involving high-dimensional geometrical parameter spaces, 


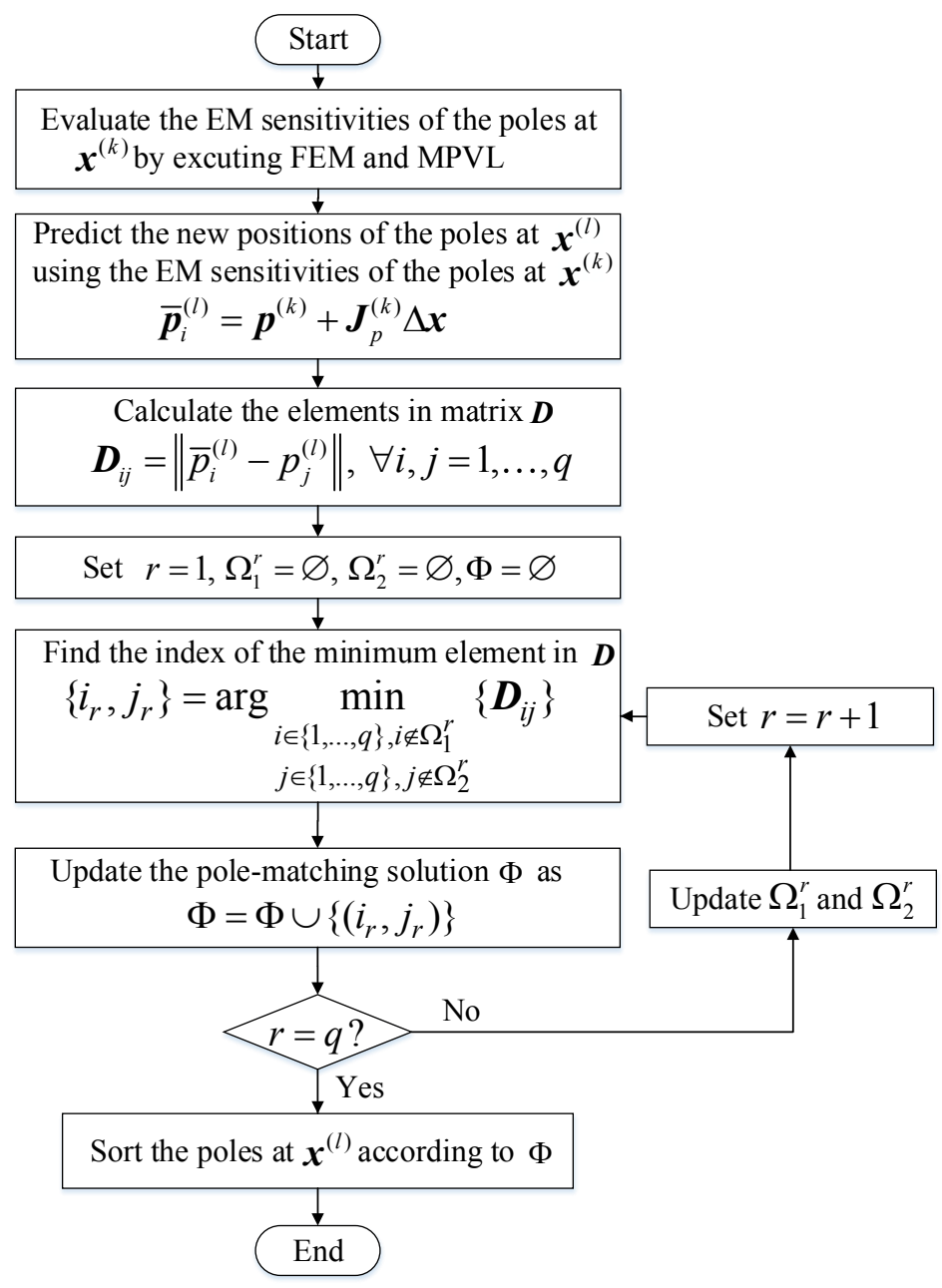

Figure 3.3: Flow chart of the proposed sensitivity-analysis-based pole-matching algorithm to match the poles between two neighboring geometrical parameter values, i.e., between $\boldsymbol{x}^{(k)}$ and $\boldsymbol{x}^{(l)}$.

the geometrical parameter values in $X$ are not necessarily grid samples. For non-grid sampling methods, the samples are scattered in different directions in the multidimensional parameter space. In such a case, it may not be possible to have a sequence of geometrical parameter values where consecutive samples are always 
within each other's neighborhood. In other words, two consecutive geometrical samples in $X$ may sometimes be far apart in the geometrical parameter space. Consequently, the data of poles at such consecutive geometrical samples may not have enough information for reliable pole-matching between them.

To solve this problem, we develop a new algorithm to enumerate all the geometrical samples in $X$ into a sequence of pairs, while ensuring that each pair of geometrical samples chosen for pole-matching are always within each other's neighborhood. The enumerating algorithm also guarantees that the poles at all the geometrical samples are matched following a common set of reference indices.

We first define two sets $\Gamma$ and $\Upsilon$, with $\Gamma \cup \Upsilon=X$. The set $\Gamma$ stores the geometrical parameter values at which the poles are already matched, while the set $\Upsilon$ stores the geometrical parameter values at which the poles are not matched yet. Initially, all the poles at all the geometrical parameter values in $X$ are not matched, thus $\Gamma=\emptyset$ and $\Upsilon=X$. To start the overall pole-matching process, we place $\boldsymbol{x}^{(0)}$ into $\Gamma$, and remove $\boldsymbol{x}^{(0)}$ from $\Upsilon$ as follows:

$$
\begin{gathered}
\Gamma=\left\{\boldsymbol{x}^{(0)}\right\}, \\
\Upsilon=X \backslash\left\{\boldsymbol{x}^{(0)}\right\},
\end{gathered}
$$

where the symbol \is used to denote the difference between two sets. Note that by using (3.12) and (3.13), the indices of the poles at $\boldsymbol{x}^{(0)}$ are designated as the common reference indices to be followed by the poles at all the other geometrical parameter values in $X$. In other words, the indices of the poles at $\boldsymbol{x}^{(0)}$ are chosen as the reference indices according to which the poles at all the other geometrical 
parameter values should be sorted. For notational convenience, let $\boldsymbol{u}$ and $\boldsymbol{v}$ denote two samples of $\boldsymbol{x}$, one from $\Gamma$ and the other from $\Upsilon$, respectively. Let $S$ be a set containing all the pairs of $\boldsymbol{u}$ and $\boldsymbol{v}$ as follows:

$$
S=\{(\boldsymbol{u}, \boldsymbol{v}) \mid \forall \boldsymbol{u} \in \Gamma, \forall \boldsymbol{v} \in \Upsilon,\|\boldsymbol{u}-\boldsymbol{v}\| \leq \delta\}
$$

where $\delta$ is the threshold for a neighborhood. According to (3.14), the $\boldsymbol{u}$ and $\boldsymbol{v}$ for each pair in $S$ are within each other's neighborhood. Once $S$ is obtained, we sort all the pairs in $S$ in ascending order according to the distance between the two members (i.e., the distance between $\boldsymbol{u}$ and $\boldsymbol{v}$ ) in each pair. Whenever a new pair is added to $S$, we compare the distance between $\boldsymbol{u}$ and $\boldsymbol{v}$ in the new pair with the distances in all the existing pairs in $S$. Then, we insert the new pair into $S$ at a suitable location according to ascending value of the distances. In this way, the first pair in $S$ always has the shortest distance between its two members among all the pairs in $S$.

Next, the first pair of $\boldsymbol{u}$ and $\boldsymbol{v}$ in $S$ will be chosen as the $\boldsymbol{x}^{(k)}$ and $\boldsymbol{x}^{(l)}$ to be used in the pole-matching process, i.e.,

$$
\left(\boldsymbol{x}^{(k)}, \boldsymbol{x}^{(l)}\right)=\left(\boldsymbol{u}_{1}, \boldsymbol{v}_{1}\right)
$$

where $\left(\boldsymbol{u}_{1}, \boldsymbol{v}_{1}\right)$ represents the first pair in $S$. Given the chosen $\boldsymbol{x}^{(k)}$ and $\boldsymbol{x}^{(l)}$, the poles between them are matched using the sensitivity-analysis-based pole-matching algorithm as described in (3.6)-(3.11). Once the matching process for these two sets 
of poles has been finished, we update $S$ by removing the pair $\left(\boldsymbol{u}_{1}, \boldsymbol{v}_{1}\right)$ as follows:

$$
S=S \backslash\left\{\left(\boldsymbol{u}_{1}, \boldsymbol{v}_{1}\right)\right\}
$$

After that, we mark the poles at $\boldsymbol{x}^{(l)}$ as already matched and then update $\Gamma$ and $\Upsilon$ as follows:

$$
\begin{aligned}
& \Gamma=\Gamma \cup\left\{\boldsymbol{x}^{(l)}\right\}, \\
& \Upsilon=\Upsilon \backslash\left\{\boldsymbol{x}^{(l)}\right\} .
\end{aligned}
$$

Lastly, we add several new pairs into $S$ as follows:

$$
S=S \cup\left\{\left(\boldsymbol{x}^{(l)}, \boldsymbol{v}\right) \mid \forall \boldsymbol{v} \in \Upsilon,\left\|\boldsymbol{x}^{(l)}-\boldsymbol{v}\right\| \leq \delta\right\}
$$

Note that the new pairs are inserted into $S$ at suitable locations so that the first pair still has the shortest distance between its two members among all the pairs in $S$. This concludes one iteration. Next, we go back to (3.15) to start the next iteration, in which a new pair of geometrical parameter values will be chosen for pole-matching.

The above procedure proceeds iteratively and terminates when $\Gamma=X$ and $\Upsilon=\emptyset$. In other words, the iterative process terminates when the poles at all the geometrical parameter values are matched already. Fig. 3.4 shows the flow chart of the overall pole-matching process. The overall matching process for the zeros can be done in a very similar manner as the matching process for the poles. The only difference is that the number of zeros at each geometrical parameter value is $q-1$ instead of $q$. After the poles and zeros at all the geometrical parameter values are 


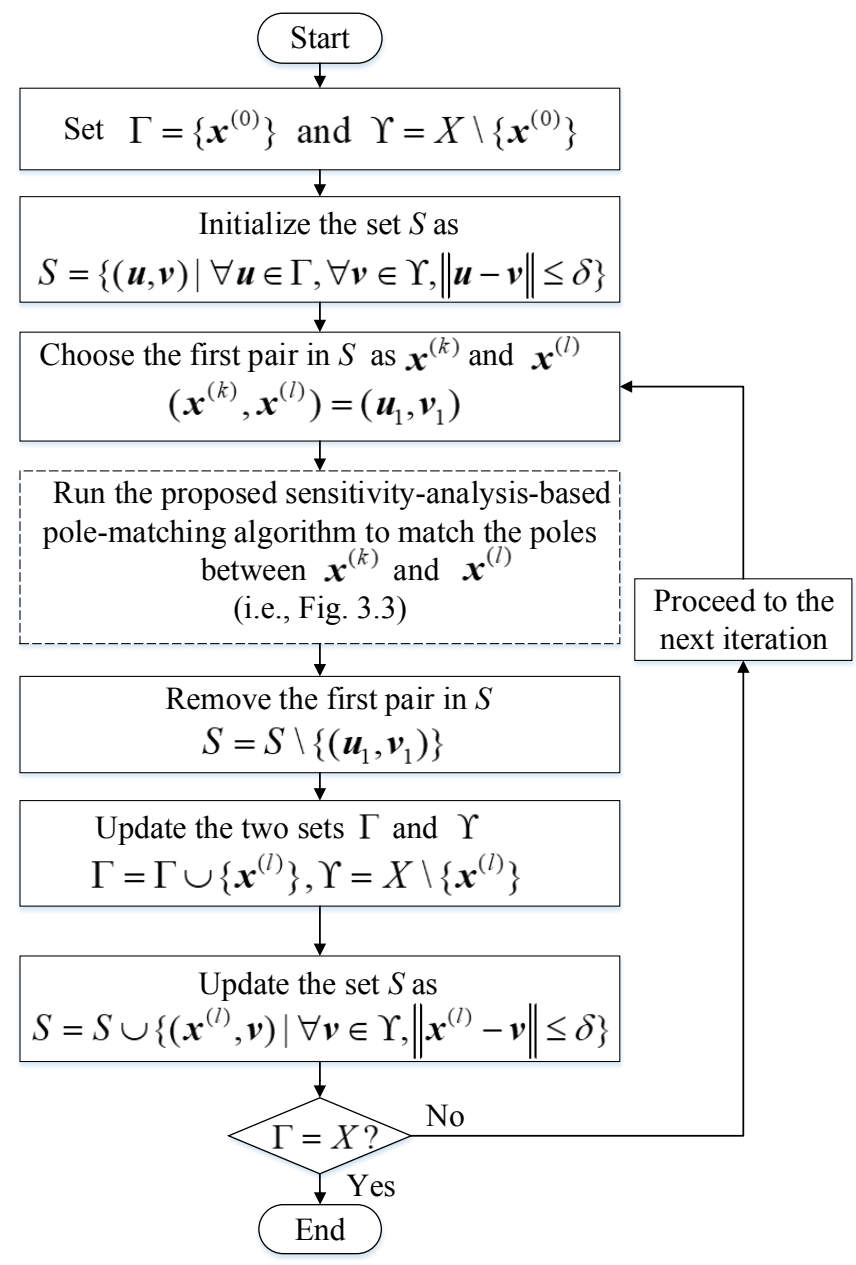

Figure 3.4: Flow chart of the algorithm to enumerate all the scattered geometrical parameter samples into pairs and to organize the sequence of pairs for matching the poles following a common set of reference indices for all the geometrical samples. The details in the dashed block is described by Fig. 3.3.

matched, we are ready to use them as training data to train the neural networks in the proposed neuro-TF model. 


\subsubsection{Preliminary Training of the Neural Networks}

The training process of the proposed neuro-TF model consists of two stages. In the first stage, we perform preliminary training of the neural networks to learn the relationships between the poles/zeros/gain and the geometrical parameters. Let the training data for this stage be denoted as $\left\{\boldsymbol{x}^{(n)}, \boldsymbol{p}^{(n)}\right\},\left\{\boldsymbol{x}^{(n)}, \boldsymbol{z}^{(n)}\right\}$, and $\left\{\boldsymbol{x}^{(n)}, K^{(n)}\right\}$, where $n \in T_{r}$. Note that the poles (zeros) used here are after being sorted using the new indices in (3.11). Since the nonlinear relationship between $\boldsymbol{p}$ and $\boldsymbol{x}$ are usually different from that between $\boldsymbol{z}$ and $\boldsymbol{x}$ and that between $K$ and $\boldsymbol{x}$, different neural networks are used to learn the relationships between $\boldsymbol{p}$ and $\boldsymbol{x}, \boldsymbol{z}$ and $\boldsymbol{x}$, and $K$ and $\boldsymbol{x}$, respectively. Let $p_{N N}, z_{N N}$, and $K_{N N}$ represent the outputs of the neural networks for poles, zeros, and gain, respectively. Let $\boldsymbol{w}_{p}, \boldsymbol{w}_{z}$, and $\boldsymbol{w}_{K}$ represent the internal neural network weights corresponding to $p_{N N}, z_{N N}$, and $K_{N N}$, respectively. The purpose in this stage is to minimize the differences between $p_{N N}\left(\boldsymbol{x}^{(n)}, \boldsymbol{w}_{p}\right)$ and $\boldsymbol{p}^{(n)}, z_{N N}\left(\boldsymbol{x}^{(n)}, \boldsymbol{w}_{z}\right)$ and $\boldsymbol{z}^{(n)}$, and $K_{N N}\left(\boldsymbol{x}^{(n)}, \boldsymbol{w}_{K}\right)$ and $K^{(n)}$, by adjusting the weights $\boldsymbol{w}_{p}, \boldsymbol{w}_{z}$, and $\boldsymbol{w}_{K}$ in a proper training process. After the preliminary training of the neural networks is finished, the overall model is further refined in the following model refinement stage.

\subsubsection{Refinement Training of the Proposed Neuro-TF Model}

In this stage, the proposed neuro-TF model is further refined in a refinement training process. The training data for this stage is $\left\{\boldsymbol{x}^{(n)}, \boldsymbol{d}^{(n)}\right\}, n \in T_{r}$, where $\boldsymbol{d}$ represents the EM responses (e.g., $S$-parameters) from EM simulation. The model inputs are geometrical parameter values and the model outputs are EM responses. The 


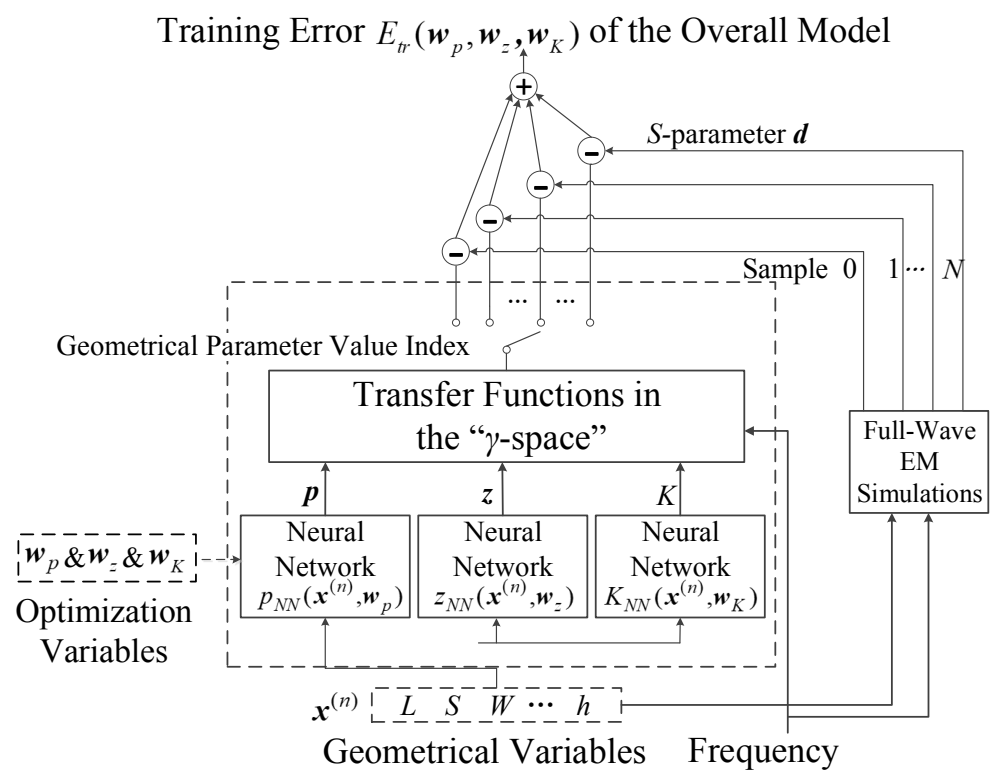

Figure 3.5: Mechanism for the refinement training process of the overall model. The optimization variables here are the weighting parameters $\boldsymbol{w}_{p}, \boldsymbol{w}_{z}$, and $\boldsymbol{w}_{K}$ in neural networks $p_{N N}, z_{N N}$, and $K_{N N}$.

mechanism for the refinement training process is illustrated in Fig. 3.5. The initial values of the neural network weights in $p_{N N}, z_{N N}$, and $K_{N N}$ are solutions from the preliminary training process in the first stage. The output vector of the overall model, $\boldsymbol{y}$, is a function of the poles, zeros, gain, and frequency, i.e.,

$$
\boldsymbol{y}=\boldsymbol{y}(\boldsymbol{p}, \boldsymbol{z}, K, \omega)
$$

where $\boldsymbol{p}, \boldsymbol{z}$, and $K$ are all functions of geometrical variables $\boldsymbol{x}$, i.e., $\boldsymbol{p}=\boldsymbol{p}(\boldsymbol{x})$, $\boldsymbol{z}=\boldsymbol{z}(\boldsymbol{x})$, and $K=K(\boldsymbol{x})$. The overall training process is to minimize the error function $E_{t r}$ by optimizing the neural network weights $\boldsymbol{w}_{p}, \boldsymbol{w}_{z}$, and $\boldsymbol{w}_{K}$ using a 
certain training algorithm:

$$
\begin{array}{r}
E_{t r}\left(\boldsymbol{w}_{p}, \boldsymbol{w}_{z}, \boldsymbol{w}_{K}\right)=\frac{1}{2(N+1)} \sum_{n \in T_{r}} \sum_{i \in F} \| \boldsymbol{y}\left(p_{N N}\left(\boldsymbol{x}^{(n)}, \boldsymbol{w}_{p}\right), z_{N N}\left(\boldsymbol{x}^{(n)}, \boldsymbol{w}_{z}\right),\right. \\
\left.K_{N N}\left(\boldsymbol{x}^{(n)}, \boldsymbol{w}_{K}\right), \omega_{i}\right)-\boldsymbol{d}_{i}^{(n)} \|^{2},
\end{array}
$$

where $T_{r}$ is the index set of all the geometrical parameter values. $i$ is the index for frequency sample and $F$ is the index set of all the frequency samples.

The training process terminates when the training error is lower than a userdefined threshold $\varepsilon$. Then, a new set of data samples which has not been used in the training process is used to test the quality of the trained model. If the testing error is also lower than $\varepsilon$, the model refinement training process terminates, and the developed model can be used for high-level circuit and system designs. Otherwise, we adjust the number of hidden neurons in $p_{N N}, z_{N N}$, and $K_{N N}$, and repeat the preliminary and refinement training processes until the desired testing error is achieved. Fig. 3.6 shows the overall flow chart of the proposed training approach for developing parametric neuro-TF models.

\subsection{Application Examples}

\subsubsection{Parametric Modeling of a Fifth-Order Waveguide Band- pass Filter: Simple Example with Two Geometrical Variables}

This example illustrates the proposed pole/zero-matching algorithm by parametric modeling of a waveguide filter with two geometrical variables. Consider a fifth-order waveguide bandpass filter with nonsymmetrical irises [98], whose structure is shown 


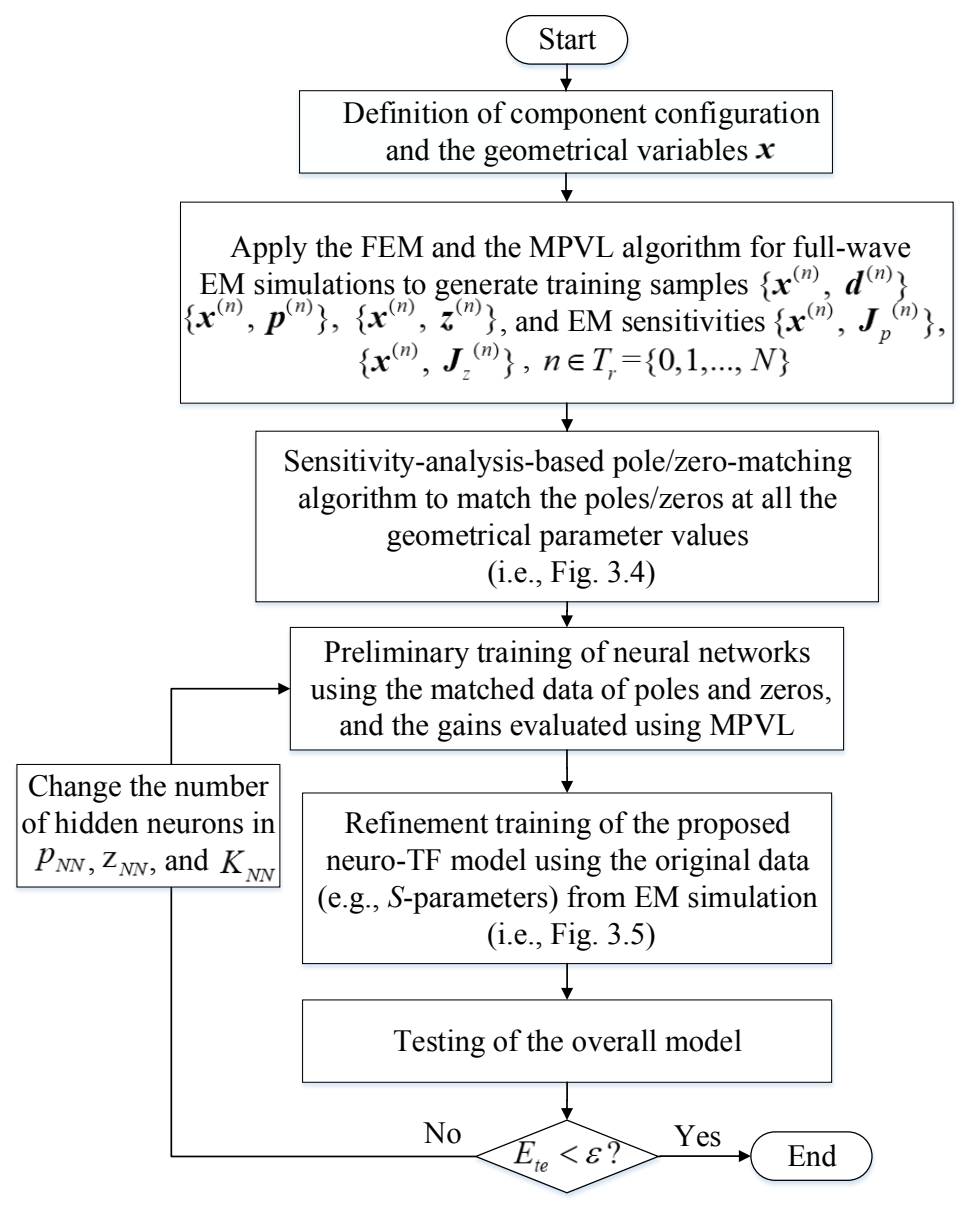

Figure 3.6: Flow chart of the overall training approach for developing parametric models of microwave passive components.

in Fig. 3.7. The cross section of the waveguide where the filter is constructed is $19.05 \mathrm{~mm} \times 9.525 \mathrm{~mm}(\mathrm{WR}-75) . d_{1}$ and $d_{2}$ are the distances from two pairs of irises to the waveguide wall. We consider $d_{1}$ and $d_{2}$ as geometrical variables. The model thus has two inputs, i.e., $\boldsymbol{x}=\left[\begin{array}{ll}d_{1} & d_{2}\end{array}\right]^{T}(\mathrm{~mm})$. Frequency is taken as an additional input to the model. The model contains one output, i.e., $\boldsymbol{y}=\left|S_{11}\right|$. 


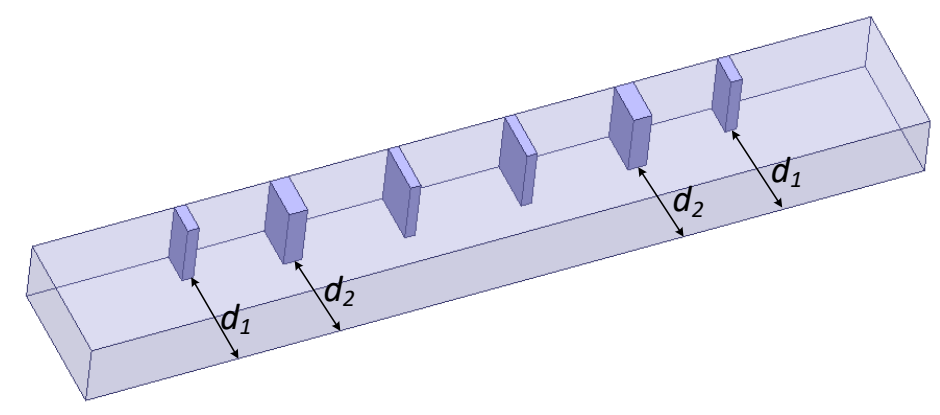

Figure 3.7: Structure of the fifth-order waveguide bandpass filter for EM simulation and parametric modeling: Simple example with two geometrical variables. For this simple example, only $d_{1}$ and $d_{2}$ are geometrical variables as inputs to the model, i.e., $\boldsymbol{x}=\left[\begin{array}{ll}d_{1} & d_{2}\end{array}\right]^{T}$.

Table 3.3: Definition of Training and Testing Data for the Fifth-Order Bandpass Filter Example with Two Geometrical Variables

\begin{tabular}{|c|c|c|c|c|c|c|c|}
\hline \multicolumn{2}{|c|}{$\begin{array}{l}\text { Geometrical } \\
\text { Variables }\end{array}$} & \multicolumn{3}{|c|}{$\begin{array}{l}\text { Training Data } \\
\text { (49 samples) }\end{array}$} & \multicolumn{3}{|c|}{$\begin{array}{l}\text { Testing Data } \\
\text { (36 samples) }\end{array}$} \\
\hline & & Min & Max & Step & Min & $\operatorname{Max}$ & Step \\
\hline \multirow{2}{*}{ Case 1} & $d_{1}(\mathrm{~mm})$ & 14.45 & 15.05 & 0.1 & 14.5 & 15.0 & 0.1 \\
\hline & $d_{2}(\mathrm{~mm})$ & 12.68 & 13.19 & 0.09 & 12.72 & 13.15 & 0.09 \\
\hline \multirow{2}{*}{ Case 2} & $d_{1}(\mathrm{~mm})$ & 13.57 & 15.92 & 0.39 & 13.76 & 15.73 & 0.39 \\
\hline & $d_{2}(\mathrm{~mm})$ & 11.9 & 13.97 & 0.35 & 12.07 & 13.8 & 0.35 \\
\hline
\end{tabular}

Since existing commercial EM simulators such as HFSS and CST do not provide the poles/zeros/gain in the " $\gamma$-space" for training the neural networks, we develop an in-house FEM program based on Gmsh [99] and an MPVL program in MATLAB to obtain the poles/zeros/gain in the " $\gamma$-space" and the EM responses of microwave passive components. In our program, the FEM is applied to solve 
the EM wave propagation problem in waveguides in the frequency domain, and the MPVL algorithm is applied to solve the FEM system for a fast frequency sweep. Mesh deformation [100][101] is incorporated into the EM simulations to guarantee the order consistency of the full-order models resulting from FEM as the values of geometrical parameters change. Appendix provides the specific equations used for the development of our program. The training and testing data for developing the overall model, the poles/zeros for training the neural networks, and the EM sensitivities for running the proposed sensitivity-analysis-based pole/zero-matching algorithm are all generated from our in-house FEM program. Grid sampling method is used in the $d_{1}-d_{2}$ space to generate both training and testing data for this example. For the MPVL algorithm, the order of the ROMs is set as $q=18$ after performing a convergence analysis of the EM responses. This results in a total of 18 poles and 17 zeros at each geometrical parameter value. The expansion point of the frequency is chosen as $11 \mathrm{GHz}$, which is the center frequency in the whole frequency range of interest (9 GHz-13 GHz).

As shown in Table 3.3, two different cases of geometrical parameter ranges are considered for parametric modeling. In Case 1, the geometrical parameters change in a narrower range, while in Case 2 the geometrical parameters change in a wider range. In both cases, seven points are sampled uniformly in the parameter range of both $d_{1}$ and $d_{2}$ to generate a total of 49 training data samples $(N+1=49)$. The poles and zeros, along with their EM sensitivities are evaluated by executing the FEM, the MPVL algorithm, and performing EM sensitivity analysis at all the geometrical parameter values (i.e., all the 49 samples). Six points are sampled uni- 
formly in the parameter range for each of the two parameters to generate a total of 36 testing data samples. The ranges of training and testing data are given in Table 3.3. The proposed sensitivity-ananlysis-based pole/zero-matching algorithm is applied. The overall neuro-TF model is trained and tested using the NeuroModelerPlus software. Using the proposed approach, in Case 1, the average training and testing errors of the model are $0.06 \%$ and $0.05 \%$, respectively, while in Case 2 , the average training and testing errors are $0.65 \%$ and $0.64 \%$, respectively.

For comparison purpose, we also apply the two existing pole-matching methods, i.e., the distance-based method and the continuation method, to match the poles and zeros at all the geometrical parameter values for both cases. For Case 1, since the geometrical parameters have small variations, both of the two existing methods can achieve correct pole/zero-matching solutions (which are the same with that of the proposed algorithm). For Case 2, however, the two existing methods perform differently from the proposed algorithm. For the purpose of illustration, we choose three poles $\left(p_{1}, p_{2}\right.$, and $\left.p_{4}\right)$ and show their original distributions in the " $\gamma$-space" in Fig. 3.8. These poles are evaluated using MPVL at each of the 49 geometrical parameter values in the training data.

The pole-matching solutions for these three selected poles using the three methods are shown in Fig. 3.9. It is observed that the three methods have different pole-matching solutions. The detailed pole-matching solutions for the first pole $p_{1}$ with the three methods are shown in Fig. 3.10, where the testing samples of $p_{1}$ are also shown explicitly. It can be seen that, by using the proposed sensitivityanalysis-based pole-matching algorithm, the pattern formed by the training samples 


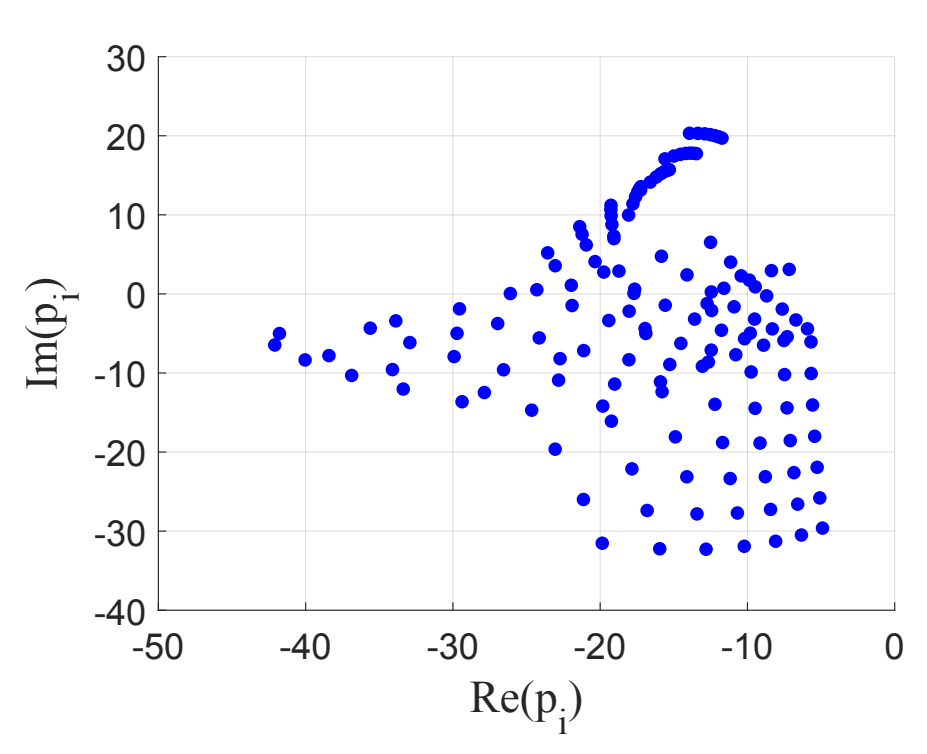

Figure 3.8: Original distributions of the three selected poles $\left(p_{1}, p_{2}\right.$, and $\left.p_{4}\right)$ in the " $\gamma$-space". These poles are evaluated using MPVL at 49 geometrical parameter samples in the training data for Case 2 of the fifth-order waveguide bandpass filter. There are a total of 147 poles (three groups with each group having 49 poles) in this figure. From these unsorted poles, we need to use a pole-matching method to identify which 49 poles belong to $p_{1}$, which 49 poles belong to $p_{2}$, and which 49 poles belong to $p_{4}$, by sorting the poles at each of the 49 geometrical parameter values correctly.

of $p_{1}$ appear to be very similar to that formed by the testing samples of $p_{1}$. When the training and testing samples of $p_{1}$ have similar patterns, one can use $p_{1}$ in the training data to predict $p_{1}$ for the testing data reliably. In contrast, by using the two existing methods, the patterns of the training and testing samples of $p_{1}$ appear to be different. This indicates that using $p_{1}$ in the training data to predict $p_{1}$ for the testing data will have large prediction errors. Similar conclusions can be drawn for the other two selected poles $p_{2}$ and $p_{4}$, as well as the zeros in the zero-matching process. In comparison with the existing methods, the proposed pole/zero-matching 


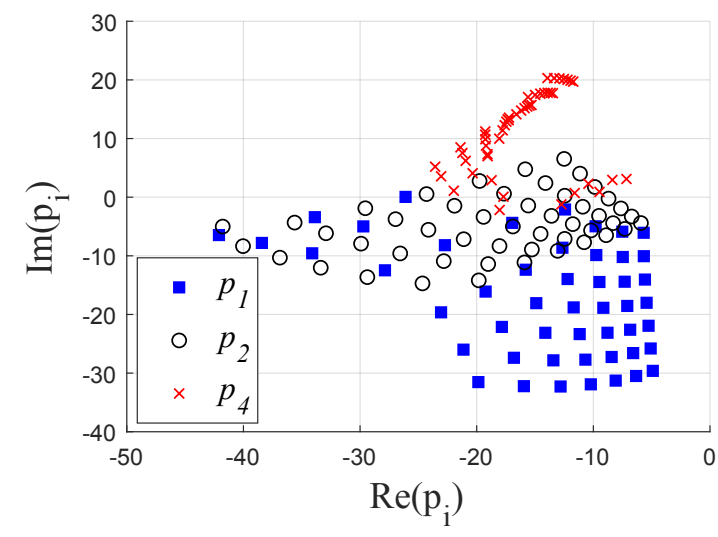

(a)

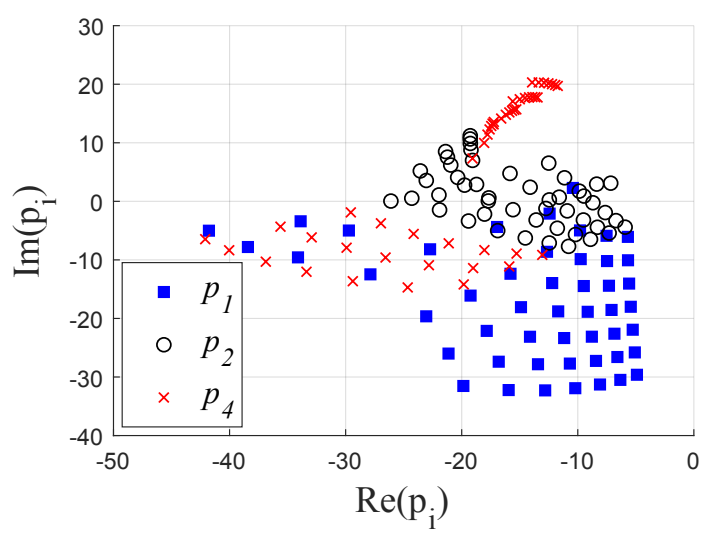

(b)

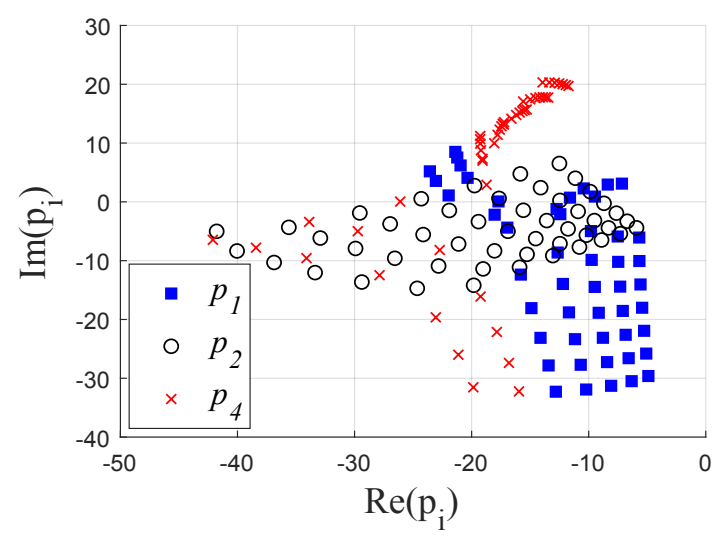

(c)

Figure 3.9: Pole-matching solutions of the three selected poles with different methods in Case 2 of the fifth-order waveguide bandpass filter example. The figure shows how the 147 poles in Fig. 3.8 are sorted and grouped into $p_{1}$ (solid squares), $p_{2}$ (circles), and $p_{4}$ (crosses) using different pole-matching methods: (a) The proposed sensitivity-analysis-based pole-matching algorithm, (b) The distance-based method, and (c) The continuation method. It is seen that different methods have different pole-matching solutions. 


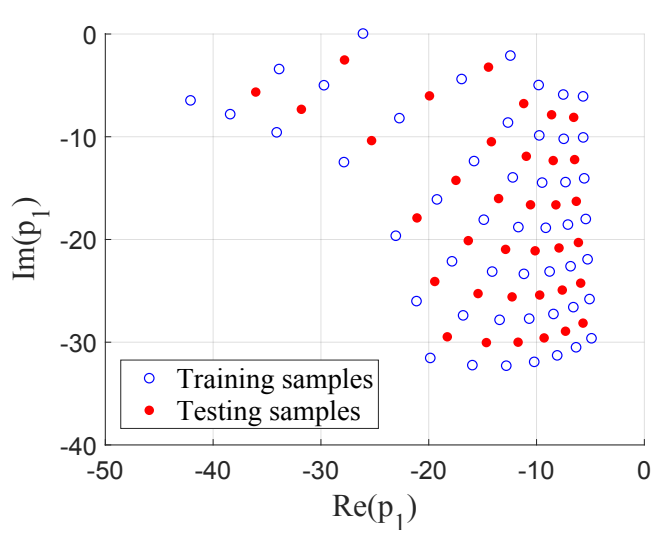

(a)

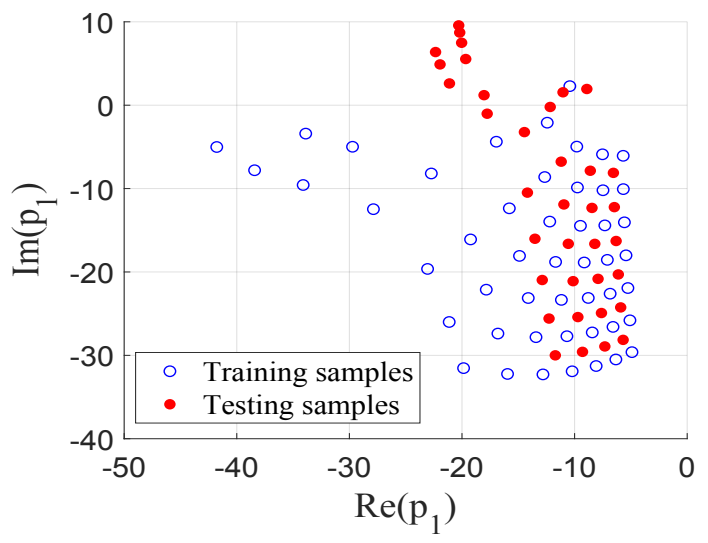

(b)

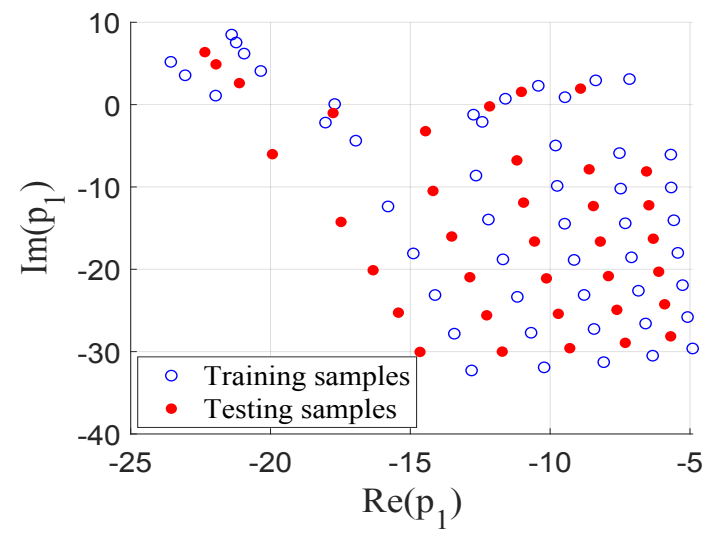

(c)

Figure 3.10: Detailed pole-matching solution for $p_{1}$ showing the degree of similarity of the patterns between training samples and testing samples for the fifth-order waveguide bandpass filter example: (a) The proposed sensitivity-analysis-based pole-matching algorithm, (b) The distance-based method, and (c) The continuation method. The training and testing samples of $p_{1}$ are represented by hollow and solid circles, respectively. 
Table 3.4: Model Accuracy Comparisons of Different Modeling Methods for the Fifth-Order Bandpass Filter Example with Two Geometrical Variables

\begin{tabular}{l|l|l|l|l}
\hline \hline \multicolumn{2}{l|}{ Modeling Method } & $\begin{array}{l}\text { Number } \\
\text { of Hidden } \\
\text { Neurons }\end{array}$ & $\begin{array}{l}\text { Average } \\
\text { Training } \\
\text { Error }\end{array}$ & $\begin{array}{l}\text { Average } \\
\text { Testing } \\
\text { Error }\end{array}$ \\
\hline \multirow{4}{*}{ Case 1 } & $\begin{array}{l}\text { Distance-based method } \\
\text { Distance-based method } \\
\text { with refinement training }\end{array}$ & 10 & $0.35 \%$ & $0.27 \%$ \\
\cline { 2 - 5 } & $\begin{array}{l}\text { Continuation method } \\
\text { with refinement training }\end{array}$ & 8 & $0.06 \%$ & $0.05 \%$ \\
\cline { 2 - 5 } & $\begin{array}{l}\text { Proposed approach } \\
\text { Case 2 }\end{array}$ & 8 & $0.06 \%$ & $0.05 \%$ \\
\cline { 2 - 5 } & $\begin{array}{l}\text { Distance-based method } \\
\text { with refinement training }\end{array}$ & 20 & $0.06 \%$ & $0.05 \%$ \\
\cline { 2 - 5 } & $\begin{array}{l}\text { Continuation method } \\
\text { with refinement training }\end{array}$ & 10 & $21.3 \%$ & $565 \%$ \\
\cline { 2 - 5 } & Proposed approach & 10 & $0.57 \%$ & $94.81 \%$ \\
\hline \hline
\end{tabular}

algorithm achieves more reliable predictions of the poles/zeros using limited EM samples in large modeling range (i.e., Case 2). This advantage will ultimately make the overall model developed by the proposed approach more accurate than those developed by using the existing methods.

We next compare the performance of the proposed approach in terms of the overall model accuracy with those of the following three existing modeling methods (slight modifications are made to align them with the proposed approach): 1) The distance-based pole-matching method; ANNs are used to interpolate the poles and residues in the transfer function; 2) The distance-based pole-matching method with 
refinement training; and 3) The continuation method with refinement training. For both 2) and 3), ANNs are used to interpolate the poles and zeros in the transfer function and a refinement training process is added to further refine the overall model. Table 3.4 compares these three existing modeling methods and the proposed approach in terms of the overall model accuracy. In Case 1, since the geometrical parameters have small variations, it is relatively convenient to match the poles/zeros (or poles/residues) correctly. Hence, the training and testing errors obtained by all the four methods are comparable and very small. In Case 2, the geometrical parameters have large variations and it becomes more challenging to match the poles/zeros correctly. In this case, only the proposed approach achieves a small testing error. This demonstrates that, when the geometrical variations are large, the proposed sensitivity-analysis-based pole/zero-matching algorithm can achieve more reliable pole/zero-matching solutions than the existing modeling methods. The reason for this is that EM sensitivities provide useful information of the direction of movements of the poles/zeros. Using these direction information, the proposed algorithm can predict the movements of the poles/zeros more reliably than those methods without using EM sensitivities, especially when the steps between two neighboring EM samples are large.

\subsubsection{Parametric Modeling of a Three-Pole H-Plane Filter}

In the second example, we consider the parametric modeling of a three-pole $H$-plane filter [9], as shown in Fig. 3.11. The cross section of the waveguide where the filter is constructed is $19.05 \mathrm{~mm} \times 9.525 \mathrm{~mm}(\mathrm{WR}-75)$. The model for this example 


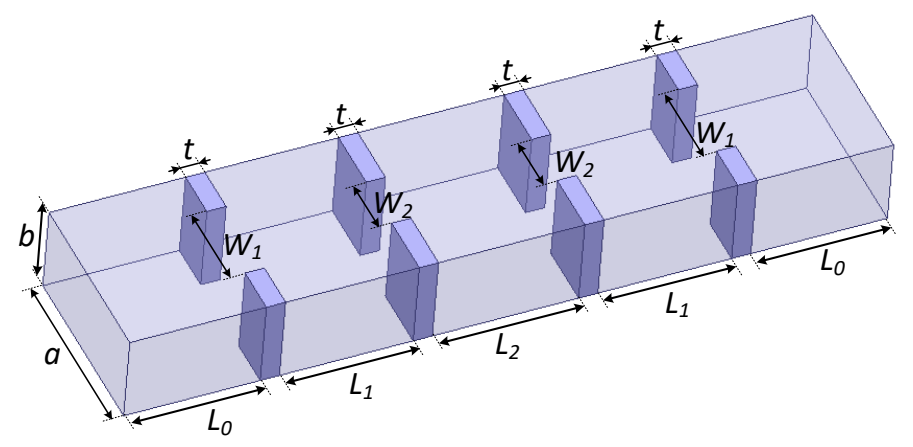

Figure 3.11: Structure of the three-pole $H$-plane filter for EM simulation and parametric modeling. The four geometrical parameters of the filter are $\boldsymbol{x}=$ $\left[\begin{array}{lllll}L_{1} & L_{2} & W_{1} & W_{2}\end{array}\right]^{T}$, with $a=19.05 \mathrm{~mm}, b=9.525 \mathrm{~mm}$, and $t=2.0 \mathrm{~mm}$.

has four geometrical variables as inputs, i.e., $\boldsymbol{x}=\left[\begin{array}{llll}L_{1} & L_{2} & W_{1} & W_{2}\end{array}\right]^{T}(\mathrm{~mm})$, and one output, i.e., $\boldsymbol{y}=\left|S_{11}\right|$, which is the magnitude of $S_{11}$. Fig. 3.12 shows the structure of the neuro-TF model for this example.

The in-house FEM program is used to generate both the training and the testing data for parametric modeling. The MPVL algorithm is applied for fast frequency sweep, with the order of the ROMs being set as $q=9$. This results in a total of 9 poles and 8 zeros at each geometrical parameter value. The expansion point of the frequency is chosen as $12 \mathrm{GHz}$, which is the center frequency in the whole frequency range of interest $(11 \mathrm{GHz}-13 \mathrm{GHz})$. We use the design of experiments (DOE) method [102] as the sampling method for both training and testing data. The DOE samples are not grid samples.

We apply the proposed approach to two different cases as defined in Table 3.5. In Case 1, the geometrical parameters change in a narrower range, while in Case 2 the geometrical parameters change in a wider range. In both cases, seven levels 


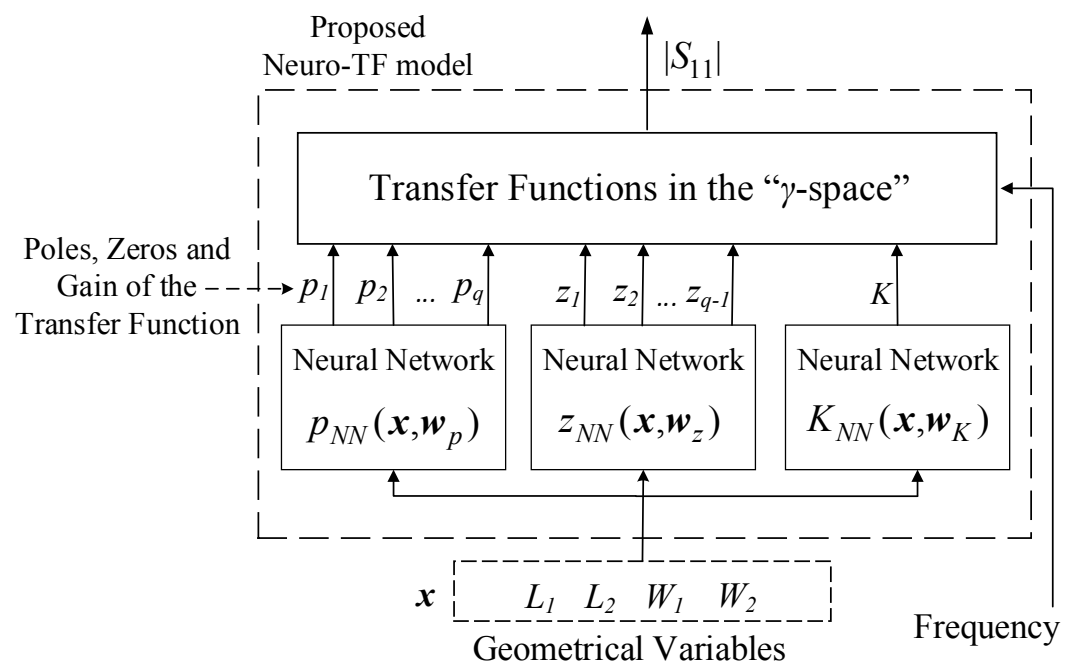

Figure 3.12: Structure of the neuro-TF model for the three-pole $H$-plane filter example.

of DOE are used for defining the samples of training data, resulting in a total of 49 samples $(N+1=49)$ of training data. Six levels of DOE are used for defining the samples of testing data, resulting in a total of 36 samples of testing data. The ranges of the training and testing data are shown in Table 3.5. Since Case 2 has larger steps between two neighboring EM samples than Case 1, it is more challenging to match the poles/zeros correctly in Case 2. For this example, the dimension of the geometrical parameter space is four. Because the EM samples generated using the DOE sampling method are not grid samples [102], it is impossible to have a sequence of 49 samples where every consecutive samples are within each other's neighborhood. We apply the algorithm described in Section 3.2.2.2 to enumerate all the geometrical parameter values for pole/zero-matching. This enumeration allows us to split the 49 geometrical parameter values into a sequence of 48 pairs. Each pair 
Table 3.5: Definition of Training and Testing Data for the Three-Pole $H$-Plane Filter Example

\begin{tabular}{|c|c|c|c|c|c|c|c|}
\hline \multirow{2}{*}{\multicolumn{2}{|c|}{$\begin{array}{c}\text { Geometrical } \\
\text { Variables }\end{array}$}} & \multicolumn{3}{|c|}{$\begin{array}{c}\text { Training Data } \\
\text { (49 samples) }\end{array}$} & \multicolumn{3}{|c|}{$\begin{array}{l}\text { Testing Data } \\
\text { (36 samples) }\end{array}$} \\
\hline & & Min & Max & Step & Min & Max & Step \\
\hline \multirow{4}{*}{ Case 1} & $L_{1}(\mathrm{~mm})$ & 13.7 & 14.26 & 0.09 & 13.75 & 14.21 & 0.09 \\
\hline & $L_{2}(\mathrm{~mm})$ & 14.9 & 15.5 & 0.1 & 14.95 & 15.45 & 0.1 \\
\hline & $W_{1}(\mathrm{~mm})$ & 8.82 & 9.18 & 0.06 & 8.85 & 9.15 & 0.06 \\
\hline & $W_{2}(\mathrm{~mm})$ & 5.88 & 6.12 & 0.04 & 5.9 & 6.1 & 0.04 \\
\hline \multirow{4}{*}{ Case 2} & $L_{1}(\mathrm{~mm})$ & 13.28 & 14.68 & 0.23 & 13.4 & 14.56 & 0.23 \\
\hline & $L_{2}(\mathrm{~mm})$ & 14.44 & 15.96 & 0.25 & 14.57 & 15.83 & 0.25 \\
\hline & $W_{1}(\mathrm{~mm})$ & 8.55 & 9.45 & 0.15 & 8.63 & 9.38 & 0.15 \\
\hline & $W_{2}(\mathrm{~mm})$ & 5.7 & 6.3 & 0.1 & 5.75 & 6.25 & 0.1 \\
\hline
\end{tabular}

of geometrical parameter values are within each other's neighborhood. Then, the algorithm presented in Section 3.2.2.1 is executed to match the 9 poles (or 8 zeros) at one geometrical parameter value with those at the other geometrical parameter value for each of the 48 pairs of geometrical parameter values. Using the proposed approach, in Case 1, the average training and testing errors are $0.16 \%$ and $0.18 \%$, respectively, while in Case 2, the average training and testing errors are $0.62 \%$ and $1.56 \%$, respectively.

For comparison purpose, we also apply the three existing modeling methods to model the EM behavior of the filter for the two cases. Table 3.6 compares the proposed approach with the three existing methods in terms of the overall model accuracy. In Case 1 , since the geometrical parameters have small variations, it is 
Table 3.6: Model Accuracy Comparisons of Different Modeling Methods for the Three-Pole $H$-Plane Filter Example

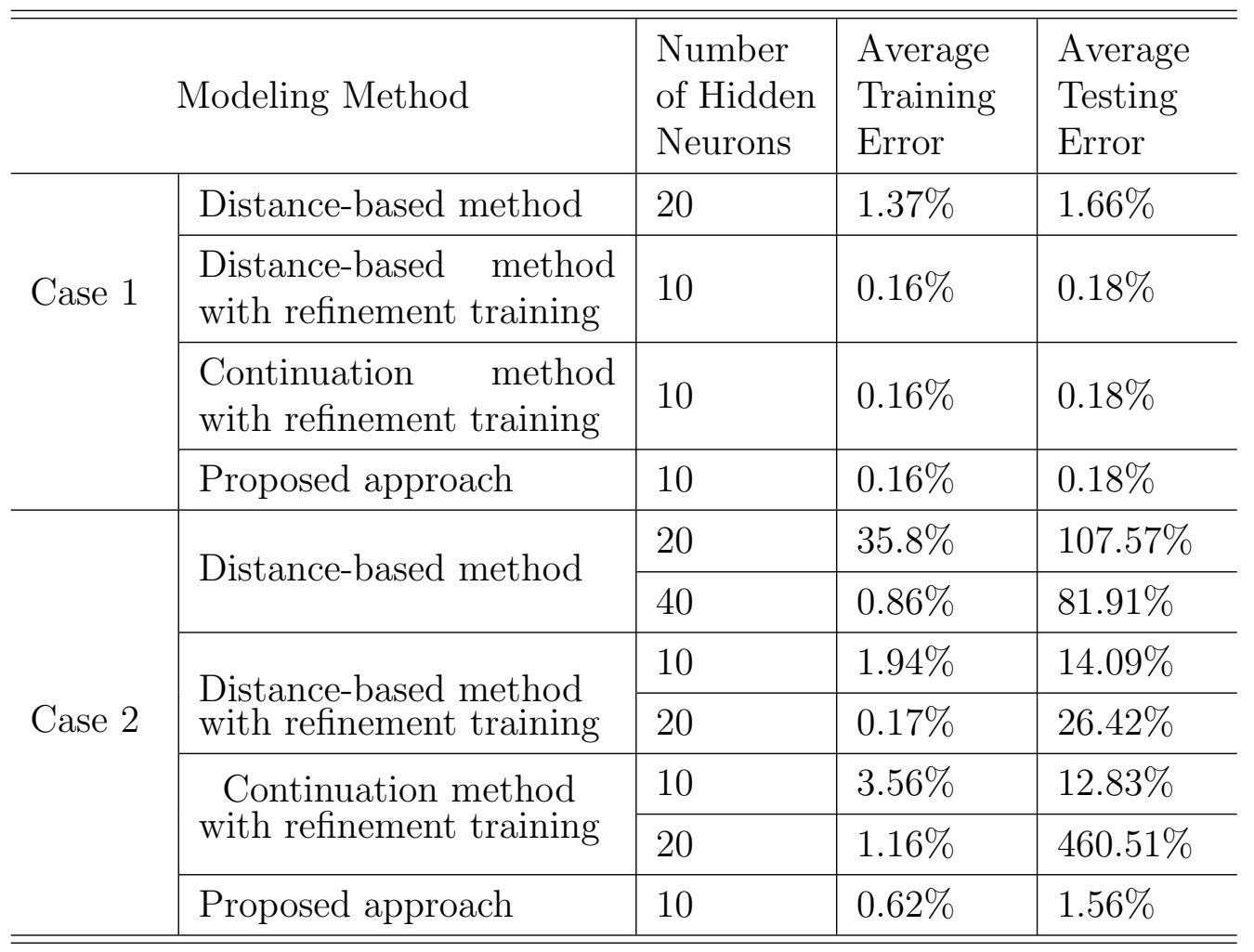

relatively convenient to match the poles/zeros (or poles/residues) correctly. All the methods achieve comparably small training and testing errors, with the distancebased method having slightly larger training and testing errors than the other three methods. The proposed approach, the distance-based method with refinement training, and the continuation method with refinement training have exactly the same training and testing errors because the pole/zero-matching solutions are identical for all the three methods. This implies that when the geometrical variations are small, all the three pole-matching methods are able to match the poles and zeros 
correctly. In Case 2, the geometrical parameters vary in a large range and the mismatch pattern of poles/zeros becomes more complex. In this case, only the proposed approach can still achieve small training and testing errors, while all the three existing methods have large testing errors. Figure 3.13 compares the model outputs obtained from the proposed approach with EM data and the model outputs obtained from the three existing methods for Case 2. This comparison is done at three different test geometrical parameter values (referred to as test geometrical parameter values $\# 1, \# 2$, and $\# 3$ ). The values of geometrical variables for the three selected test samples of filters are as follows:

Test geometrical parameter value

\#1: $\boldsymbol{x}=\left[\begin{array}{llll}14.1742 & 14.9889 & 9 & 5.8333\end{array}\right]^{T}(\mathrm{~mm})$

Test geometrical parameter value

$\# 2: \boldsymbol{x}=\left[\begin{array}{lll}14.1742 & 15.62228 .875 & 6.16667\end{array}\right]^{T}(\mathrm{~mm})$

Test geometrical parameter value

\#3: $\boldsymbol{x}=\left[\begin{array}{llll}13.7858 & 14.9889 & 9.125 & 6.25\end{array}\right]^{T}(\mathrm{~mm})$

It can be observed from Fig. 3.13 that, when the geometrical variations are large, the model outputs obtained from the proposed approach match the EM data much better than the model outputs obtained from the other three existing methods. Moreover, it can be concluded that the proposed model is accurate at the test values even though these test values are not used in the training process. 


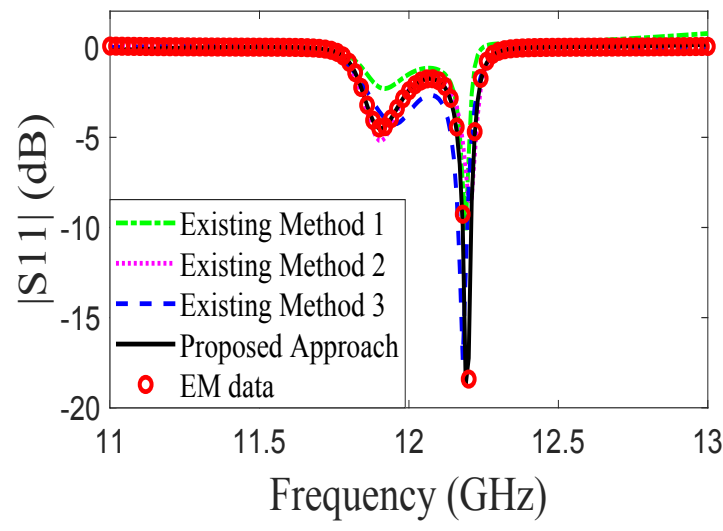

(a)

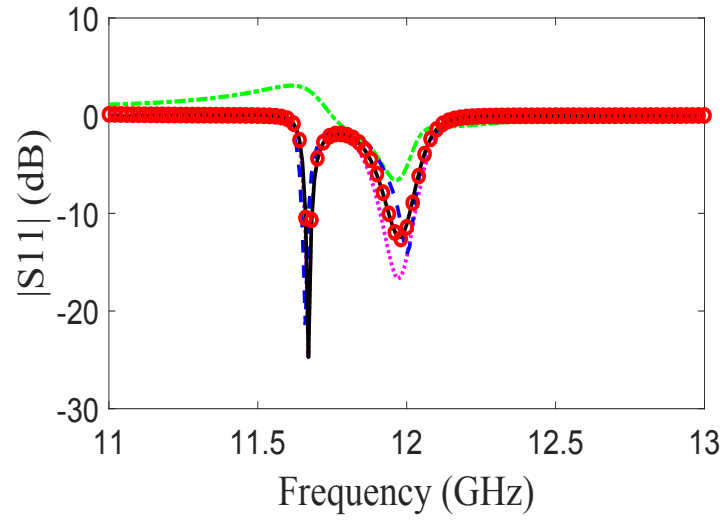

(b)

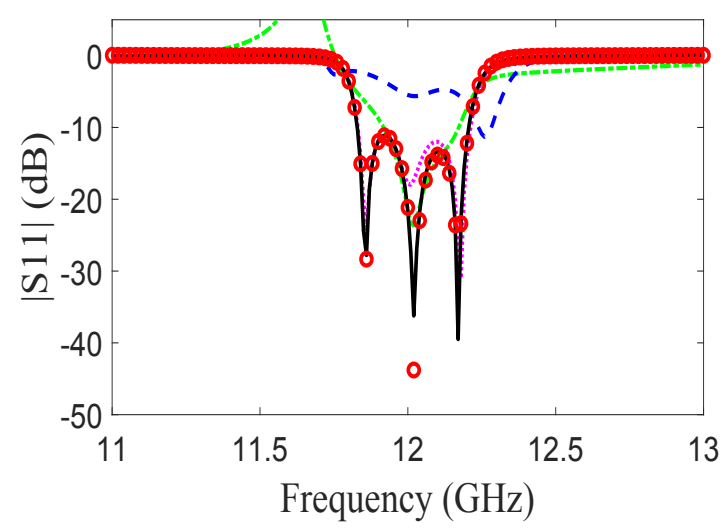

(c)

Figure 3.13: Comparison of the outputs ( $\left|S_{11}\right|$ in decibels) of the models and the EM data for Case 2 of the three-pole $H$-plane filter example: (a) Test geometrical parameter value \#1, (b) Test geometrical parameter value \#2, and (c) Test geometrical parameter value \#3. The three existing methods are: 1) Distance-based method, 2) Distance-based method with refinement training, and 3) Continuation method with refinement training. The model outputs obtained from the proposed approach matches the EM data much better than the model outputs obtained from the three existing methods. 


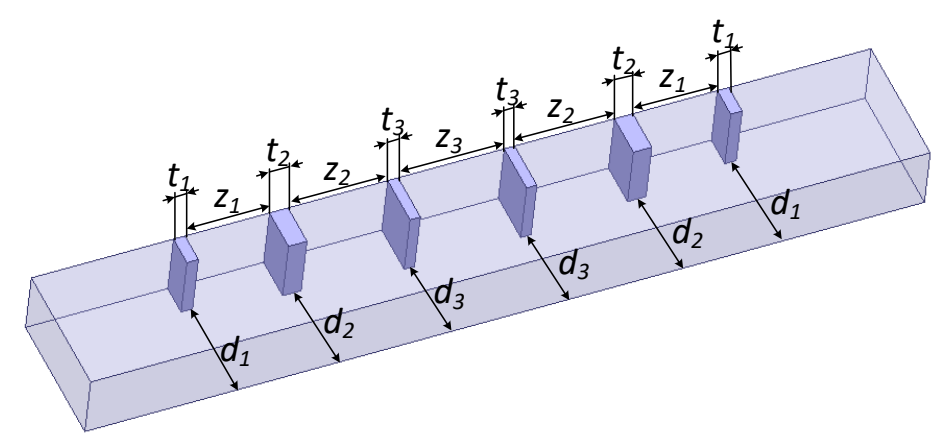

Figure 3.14: Structure of fifth-order waveguide bandpass filter for EM simulation and parametric modeling: Harder example with nine geometrical variables. The

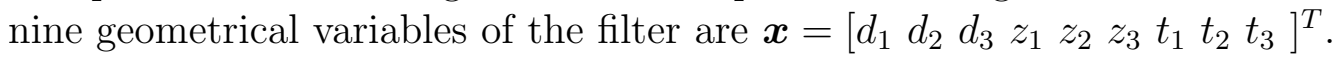

\subsubsection{Parametric Modeling of the Fifth-Order Waveguide Bandpass Filter: Harder Example with Nine Geomet- rical Variables}

In this example, we consider a harder case of parametric modeling of the fifthorder waveguide bandpass filter. The structure of the filter is shown in Fig. 3.14. $d_{1}, d_{2}$, and $d_{3}$ are the distances from the irises to the waveguide wall. $z_{1}, z_{2}$, and $z_{3}$ are the distances between two adjacent irises. The thicknesses of the irises are $t_{1}, t_{2}$, and $t_{3}$. The model has nine geometrical variables as inputs, i.e., $\boldsymbol{x}=$

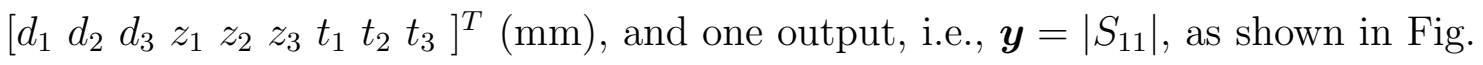

3.15. The order of the reduced-order models in the MPVL algorithm is set as $q=18$. DOE method is used as the sampling method in generating training and testing data. The DOE samples are not grid samples.

The proposed approach is applied to two different cases as defined in Table 3.7. In Case 1, the geometrical parameters change in a narrower range, while in Case 


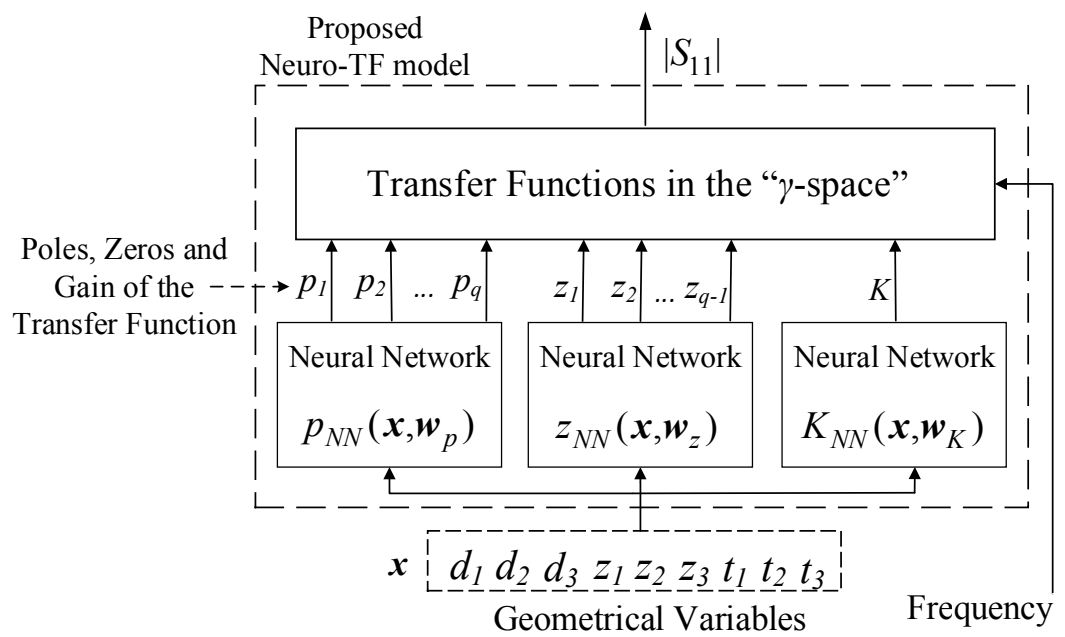

Figure 3.15: Structure of the neuro-TF model for the fifth-order waveguide bandpass filter example with nine geometrical variables.

2 the geometrical parameters change in a wider range. In both cases, nine levels of DOE are used for defining the training data, resulting in a total of 81 samples $(N+1=81)$ of training data, while eight levels of DOE are used for defining the testing data, resulting in a total of 64 samples of testing data. Since Case 2 has larger steps between two neighboring EM samples than Case 1, it is harder to match the poles/zeros correctly in Case 2. For this example, the dimension of the geometrical parameter space is nine. The EM samples generated using the DOE sampling method are not grid samples [102]. Therefore, it is not possible to have a sequence of 81 samples where every consecutive samples are within each other's neighborhood. We apply the algorithm described in Section 3.2.2.2 to enumerate all the geometrical parameter values for pole/zero-matching. This enumeration allows us to split the 81 geometrical parameter values into a sequence of 80 pairs. Each pair of geometrical parameter values are within each other's neighborhood. Then, 
the algorithm presented in Section 3.2.2.1 is executed to match the 18 poles (or 17 zeros) at one geometrical parameter value with those at the other geometrical parameter value for each of the 80 pairs of geometrical parameter values. Using the proposed modeling technique, in Case 1, the average training and testing errors of the model are $0.08 \%$ and $0.07 \%$, respectively, while in Case 2, the average training and testing errors of the model are $0.78 \%$ and $1.59 \%$, respectively.

For comparison purpose, the three existing modeling methods are also applied to the two cases. Table 3.8 compares the three existing methods and the proposed approach in terms of the overall model accuracy. In Case 1, the geometrical variations are small and the issue of mismatch of poles and zeros is relatively convenient to address. All the four methods in the comparison achieve small training and testing errors. The models developed by the proposed approach, the distancebased method with refinement training, and the continuation method with refinement training have the same model accuracy because the pole/zero-matching solutions obtained from the three methods are exactly the same. This is because when geometrical variations are small, both the proposed sensitivity-analysis-based pole/zero-matching algorithm and the two existing pole-matching methods are able to match the poles/zeros correctly. In Case 2, since the geometrical parameters have large variations, the mismatch pattern of poles/zeros becomes more complex. The proposed approach can still achieve small training and testing errors, while all the other three existing methods have large testing errors. It is also noticeable that the proposed approach can achieve a smaller training error with robust ANN structures than all the three existing methods. 


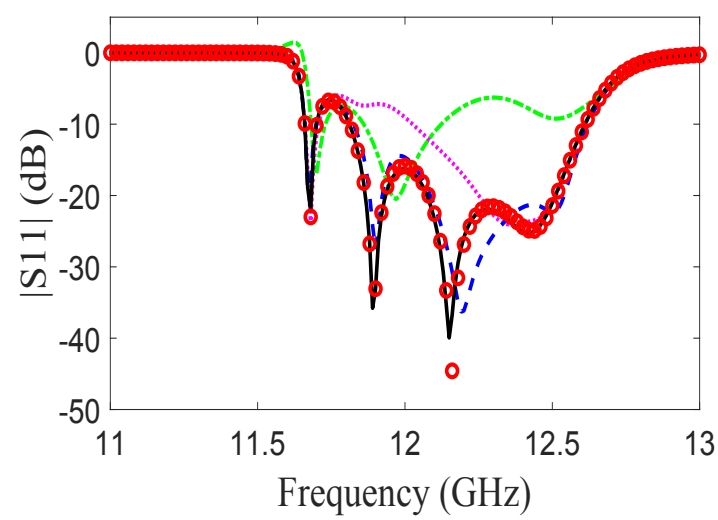

(a)

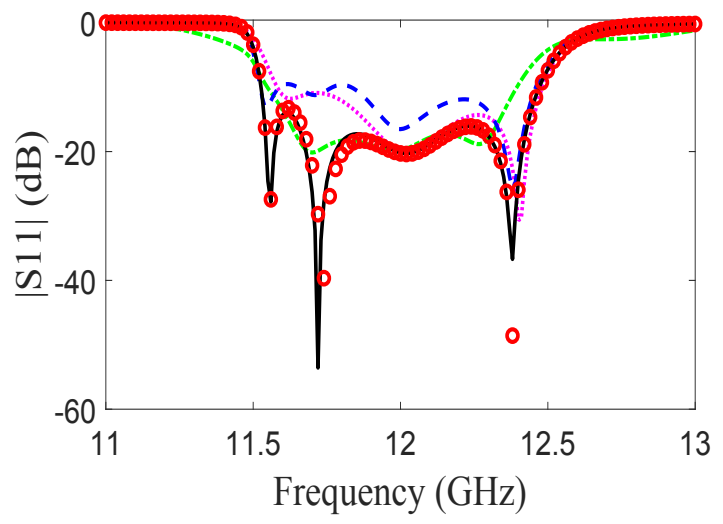

(b)

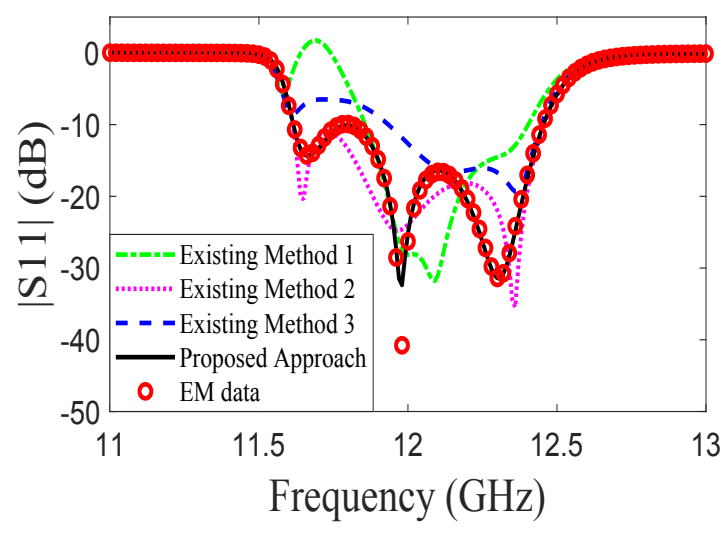

(c)

Figure 3.16: Comparison of the outputs ( $\left|S_{11}\right|$ in decibels) of the models and the EM data for Case 2 of the fifth-order waveguide bandpass filter example: (a) Test geometrical parameter value \#1, (b) Test geometrical parameter value \#2, and (c) Test geometrical parameter value \#3. The three existing methods are: 1) Distance-based method, 2) Distance-based method with refinement training, and 3) Continuation method with refinement training. It can be seen that the model outputs obtained from the proposed approach matches with the EM data much better than the model outputs obtained from the three existing methods. 
Table 3.7: Definition of Training and Testing Data for the Fifth-Order Bandpass Filter Example with Nine Geometrical Variables

\begin{tabular}{|c|c|c|c|c|c|c|c|}
\hline \multirow{2}{*}{\multicolumn{2}{|c|}{$\begin{array}{c}\text { Geometrical } \\
\text { Variables }\end{array}$}} & \multicolumn{3}{|c|}{$\begin{array}{c}\text { Training Data } \\
\text { (81 samples) }\end{array}$} & \multicolumn{3}{|c|}{$\begin{array}{l}\text { Testing Data } \\
\text { (64 samples) }\end{array}$} \\
\hline & & Min & Max & Step & Min & Max & Step \\
\hline \multirow{9}{*}{ Case 1} & $d_{1}(\mathrm{~mm})$ & 14.6 & 14.89 & 0.04 & 14.62 & 14.87 & 0.04 \\
\hline & $d_{2}(\mathrm{~mm})$ & 12.81 & 13.07 & 0.04 & 12.83 & 13.05 & 0.04 \\
\hline & $d_{3}(\mathrm{~mm})$ & 11.76 & 12 & 0.03 & 11.78 & 11.98 & 0.03 \\
\hline & $z_{1}(\mathrm{~mm})$ & 11.76 & 12 & 0.03 & 11.78 & 11.98 & 0.03 \\
\hline & $z_{2}(\mathrm{~mm})$ & 13.89 & 14.17 & 0.04 & 13.91 & 14.15 & 0.04 \\
\hline & $z_{3}(\mathrm{~mm})$ & 14.78 & 15.08 & 0.04 & 14.81 & 15.06 & 0.04 \\
\hline & $t_{1}(\mathrm{~mm})$ & 1.59 & 1.63 & 0.005 & 1.6 & 1.63 & 0.005 \\
\hline & $t_{2}(\mathrm{~mm})$ & 2.57 & 2.63 & 0.007 & 2.58 & 2.62 & 0.007 \\
\hline & $t_{3}(\mathrm{~mm})$ & 1.43 & 1.48 & 0.004 & 1.44 & 1.47 & 0.004 \\
\hline \multirow{9}{*}{ Case 2} & $d_{1}(\mathrm{~mm})$ & 14.01 & 15.48 & 0.18 & 14.1 & 15.39 & 0.18 \\
\hline & $d_{2}(\mathrm{~mm})$ & 12.42 & 13.45 & 0.13 & 12.48 & 13.39 & 0.13 \\
\hline & $d_{3}(\mathrm{~mm})$ & 11.64 & 12.11 & 0.06 & 11.67 & 12.09 & 0.06 \\
\hline & $z_{1}(\mathrm{~mm})$ & 11.29 & 12.47 & 0.15 & 11.36 & 12.4 & 0.15 \\
\hline & $z_{2}(\mathrm{~mm})$ & 13.68 & 14.38 & 0.09 & 13.73 & 14.34 & 0.09 \\
\hline & $z_{3}(\mathrm{~mm})$ & 14.56 & 15.31 & 0.09 & 14.6 & 15.26 & 0.09 \\
\hline & $t_{1}(\mathrm{~mm})$ & 1.29 & 1.93 & 0.08 & 1.33 & 1.89 & 0.08 \\
\hline & $t_{2}(\mathrm{~mm})$ & 2.21 & 2.99 & 0.1 & 2.26 & 2.94 & 0.1 \\
\hline & $t_{3}(\mathrm{~mm})$ & 1.16 & 1.74 & 0.07 & 1.2 & 1.71 & 0.07 \\
\hline
\end{tabular}

Figure 3.16 compares the model outputs obtained from the proposed approach with EM data and the model outputs obtained from the three existing methods for Case 2. This comparison is done at three different test geometrical parameter 
Table 3.8: Model Accuracy Comparisons of Different Modeling Methods for the Fifth-Order Bandpass Filter Example with Nine Geometrical Variables

\begin{tabular}{|c|c|c|c|c|}
\hline \multicolumn{2}{|r|}{ Modeling Method } & $\begin{array}{l}\text { Number } \\
\text { of Hidden } \\
\text { Neurons }\end{array}$ & $\begin{array}{l}\text { Average } \\
\text { Training } \\
\text { Error }\end{array}$ & $\begin{array}{l}\text { Average } \\
\text { Testing } \\
\text { Error }\end{array}$ \\
\hline \multirow{4}{*}{ Case 1} & Distance-based method & 10 & $0.71 \%$ & $0.55 \%$ \\
\hline & $\begin{array}{l}\text { Distance-based method } \\
\text { with refinement training }\end{array}$ & 10 & $0.08 \%$ & $0.07 \%$ \\
\hline & $\begin{array}{l}\text { Continuation method } \\
\text { with refinement training }\end{array}$ & 10 & $0.08 \%$ & $0.07 \%$ \\
\hline & Proposed approach & 10 & $0.08 \%$ & $0.07 \%$ \\
\hline \multirow{7}{*}{ Case 2} & \multirow{2}{*}{ Distance-based method } & 10 & $28.59 \%$ & $41.81 \%$ \\
\hline & & 40 & $4.64 \%$ & $35.42 \%$ \\
\hline & \multirow{2}{*}{$\begin{array}{l}\text { Distance-based method } \\
\text { with refinement training }\end{array}$} & 10 & $2.77 \%$ & $13.61 \%$ \\
\hline & & 20 & $1.07 \%$ & $21.29 \%$ \\
\hline & \multirow{2}{*}{$\begin{array}{l}\text { Continuation method } \\
\text { with refinement training }\end{array}$} & 10 & $1.97 \%$ & $12.61 \%$ \\
\hline & & 20 & $1.61 \%$ & $54.92 \%$ \\
\hline & Proposed approach & 10 & $0.78 \%$ & $1.59 \%$ \\
\hline
\end{tabular}

values. We refer to them as test geometrical parameter values $\# 1$, \#2, and \#3. The values of geometrical variables for the three selected test samples of filters are as follows:

Test geometrical parameter value

\#1: $\boldsymbol{x}=[14.099812 .483411 .6711 .360413 .7259$

$14.60751 .330162 .258751 .19939]^{T}(\mathrm{~mm})$

Test geometrical parameter value

$\# 2: \boldsymbol{x}=[14.837113 .130212 .085711 .805914 .1645$ 
$14.79411 .410782 .843751 .19939]^{T}(\mathrm{~mm})$

Test geometrical parameter value

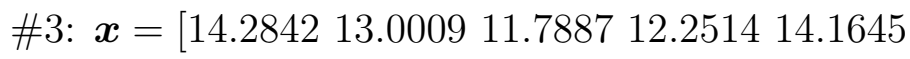

$14.88751 .330162 .648751 .70822]^{T}(\mathrm{~mm})$

It can be observed from Fig. 3.16 that, when the geometrical variations are large, the model outputs obtained from the proposed approach match the EM data much better than the model outputs obtained from the other three existing methods.

\subsection{Conclusions and Summary}

In this chapter, a novel training approach for developing parametric models of microwave passive components using Padé via Lanczos and EM sensitivities has been proposed. The EM responses (e.g., $S$-parameters) of passive components versus frequency have been represented by pole-zero-gain transfer functions. To address the issue of mismatch of poles/zeros, a novel sensitivity-analysis-based pole/zeromatching algorithm has been proposed. The proposed algorithm utilizes EM sensitivities, which provides useful information for the direction of movement of the poles/zeros, to predict the new positions of the poles/zeros for each change of geometrical parameters. The predicted new positions have been used to guide the matching process of poles/zeros between different geometrical parameter values. It has been shown that in comparison with the existing methods, the proposed algorithm can obtain more reliable pole/zero-matching solutions when geometrical variations are large. This allows the trained neural networks to have more accurate predictions for the poles and zeros subject to large geometrical variations, and ul- 
timately increases the accuracy and robustness of the overall model. It has been demonstrated that the proposed approach can obtain better accuracy in challenging applications involving large geometrical variations. 


\section{Chapter 4}

\section{Surrogate-Assisted Approach to Yield-Driven EM Optimization}

\subsection{Introduction}

Yield optimization, also called design centering, is an optimization process that aims to find a nominal design solution with the maximum yield [25]. Direct electromagnetic (EM)-based yield optimization does not appear to be feasible as a significantly large number of EM simulations are typically required during the whole optimization process [50]. As a recognized engineering optimization methodology, space mapping (SM) holds a great potential to accelerate yield-driven EM optimization [103]-[105]. The basic concept of SM is to replace EM-based fine models with fast yet not so accurate coarse models and calibrate the coarse models with certain mapping (linear or nonlinear) structures in each iteration during optimization. Such mapping structures construct a mathematical link between a coarse model and a fine model. Through SM, the original CPU-intensive evaluations on the fine model are replaced by fast computations on the coarse model, while the accuracy of the fine model is 
still maintained [103].

In the last two decades, a number of research activities have been carried out to develop SM-based approaches to facilitate yield optimization of microwave structures [28], [32], [92], [106]-[108]. For example, neural space-mapping models have been exploited in [106] to perform EM-based yield optimization efficiently. The sensitivity formulas of the surrogate model responses have also been derived, which are then used in approximating the sensitivities of the fine model responses. In [107], a tuning space-mapping surrogate based yield estimation and optimization technique has been presented. The responses of the surrogate are further corrected to obtain improved yield accuracy, facilitating the overall yield optimization process. However, in these two methods, the SM surrogate is not updated during yield optimization, which means that a large amount of EM data are required to train the surrogate to be valid in a large region. Some other research works exist in the literature that update the SM surrogate in each iteration during yield optimization. For example, in [28], a modified ellipsoidal technique has been incorporated into space mapping and then applied to the yield optimization problem of microwave circuits. The mapping structure between coarse and fine models is updated iteratively in yield optimization using the EM responses at all the nominal points in the preceding iterations. In [108], a derivative-free trust region approach has been presented to maximize the yield of microwave circuits, where the generalized SM surrogate is used and updated at each SM iteration in yield optimization. For both [28] and [108], Jacobian matrices of the fine model responses are also used in the parameter extraction process to enhance the accuracy of the surrogate model. Re- 
cently, SM approaches exploiting response features have also been investigated with the objective of achieving more efficient yield estimation and/or optimization of microwave structures [32], [92].

All the aforementioned SM-based methods do not use parallel computation, which holds a great potential to speed up EM design optimization. Parallel computation is an efficient technique to speed up EM optimization by accelerating EM data generation [33] and surrogate model training [34]. Recently, a parallel SM approach has been presented in [35], where the parallel computation mechanism has been combined with SM, to facilitate EM-based nominal optimization. It has been shown that with parallel computation, the SM surrogate can be trained to be valid in a relatively larger neighborhood and the optimal nominal design can be obtained in shorter time and fewer SM iterations [35].

Yield estimation is an indispensable component of yield optimization. Traditional Monte Carlo (MC) method for EM-based yield estimation is computationally expensive as a large number of EM simulations are required to achieve desired yield estimation accuracy. Recently, the polynomial chaos expansion (PCE) approach [36] has emerged as a powerful tool for statistical analysis and yield estimation in microwave design [37]-[95]. Existing studies have shown that PCE has significant benefits over the traditional Monte Carlo analysis in terms of reduced computational costs and shorter CPU time for yield estimation of microwave structures [95]. However, how to combine SM, the parallel computation method, and PCE to facilitate EM-based yield optimization still remain an open subject in the literature.

In this chapter, we propose a novel parallel space-mapping based yield-driven 
EM optimization technique incorporating trust region algorithm and PCE. In this technique, a novel trust region algorithm designed specifically for EM-based yielddriven design is proposed to increase the robustness of the SM surrogate. The proposed algorithm updates the trust radius of each design parameter in each SM iteration based on the effectiveness of minimizing the $l_{1}$ objective function using the surrogate. Moreover, for the first time, we incorporate the parallel computation method to SM-based yield optimization of microwave structures. Specifically, parallel computation method is used to generate fine model EM responses at multiple geometrical samples simultaneously and to train the surrogate model to match the fine model over multiple geometrical samples. The use of parallel computation allows the surrogate to to be trained in a larger neighborhood in the design parameter space than that in standard SM, consequently increasing the speed of finding the optimal yield solution in yield-driven design. Lastly, we propose to incorporate the PCE approach, which is an efficient alternative method for EM-based yield estimation, into the proposed technique to further accelerate the overall yield optimization process. Compared with the standard SM-based yield optimization technique, the proposed technique reduces the number of SM iterations to achieve a desired yield value, thereby accelerating the overall EM-based yield optimization process. Two microwave examples are presented to demonstrate the advantages of the proposed technique. 


\subsection{Formulation of the Original EM-based Yield Optimization Problem}

Let $\boldsymbol{x}$ be a $n$-dimensional vector containing $n$ design parameters (e.g., geometrical/physical parameters) of the microwave structure under consideration. Let $\boldsymbol{x}^{0}$ denote the nominal point of $\boldsymbol{x}$. In the approaches to statistical design of microwave structures, the uncertainties introduced by the manufacturing process make the actual values of $\boldsymbol{x}$ be distributed around $\boldsymbol{x}^{0}$. The distribution is usually considered to follow some kind of distribution, e.g., uniform, Gaussian, etc.

Microwave design typically involves goals in terms of a number of design specifications applied on the responses of microwave structures. In practice, the design specifications are sampled at a number of frequency points in the whole frequency bands of interest. Let $N_{s}$ denote the total number of design specification samples, which usually consist of both the upper and the lower ones. Let the $j$ th design specification sample be denoted by $S_{j}$, where $j=1, \cdots, N_{s}$. Let $N_{s}^{u}$ and $N_{s}^{l}$ $\left(N_{s}^{l}=N_{s}-N_{s}^{u}\right)$ be the number of upper specification samples and the number of lower specification samples, respectively. Without the loss of generality, we assume that the first $N_{s}^{u}$ samples, $S_{1}, \cdots, S_{N_{s}^{u}}$, represent upper design specification samples, and that the remaining $N_{s}^{l}$ samples, $S_{N_{s}^{u}+1}, \cdots, S_{N_{s}}$, are lower design specification

samples. Let $R_{j}^{f}(\boldsymbol{x})$ represent the response of the fine model at the frequency of interest corresponding to $S_{j}$. Let $\boldsymbol{e}(\boldsymbol{x})$ be an error vector used to indicate how well the response vector of the fine model satisfies the design specifications. Specifically, 
$\boldsymbol{e}(\boldsymbol{x})$ is defined as follows:

$$
\boldsymbol{e}(\boldsymbol{x})=\left[e_{1} e_{2} \cdots e_{N_{s}^{u}} \cdots e_{N_{s}}\right]^{T},
$$

where the $j$ th element in the above vector, $e_{j}(\boldsymbol{x})$, is given by

$$
e_{j}(\boldsymbol{x})= \begin{cases}R_{j}^{f}(\boldsymbol{x})-S_{j}, & \text { if } 1 \leq j \leq N_{s}^{u}, \\ S_{j}-R_{j}^{f}(\boldsymbol{x}), & \text { if } N_{s}^{u}<j \leq N_{s} .\end{cases}
$$

Yield refers to the percentage of non-defective designs of all produced designs. To realize EM-based yield analysis and optimization, a sufficiently large number of random outcomes of the design parameters are typically generated, represented by

$$
\boldsymbol{x}^{m}=\boldsymbol{x}^{0}+\Delta \boldsymbol{x}^{m}, \quad m=1,2, \ldots, N_{m c},
$$

where $\boldsymbol{x}^{m}$ represents the deviation between the $m$ th outcome and the nominal point, and $N_{m c}$ is the total number of random outcomes for yield analysis. In this chapter, $m\left(m=1, \cdots, N_{m c}\right)$ is used to represent the index of random outcomes in Monte Carlo analysis. Due to the uncertainties in the manufacturing process, the EM responses at some outcomes may satisfy design specifications while others may not. Let the yield at nominal point $\boldsymbol{x}^{0}$ be denoted as $Y\left(\boldsymbol{x}^{0}\right)$. Then, $Y\left(\boldsymbol{x}^{0}\right)$ can be approximated as the number of acceptable design outcomes over the total number of design outcomes, i.e.,

$$
Y\left(\boldsymbol{x}^{0}\right) \approx N_{\text {acpt }} / N_{m c}
$$

where $N_{\text {acpt }}$ represents the total number of acceptable design outcomes.

Let $H_{p}(\cdot)$ represent the one-sided least $p$ th function. According to [25], the 
following objective function $U\left(\boldsymbol{x}^{0}\right)$ can be used to solve the original EM-based yield optimization problem

$$
U\left(\boldsymbol{x}^{0}\right)=H_{p}\left(\boldsymbol{u}\left(\boldsymbol{x}^{0}\right)\right)
$$

where $\boldsymbol{u}=\left[u_{1}, u_{2}, \cdots, u_{N_{m c}}\right]^{T}$. The $m$ th component in $\boldsymbol{u}$ is found from

$$
u_{m}=\alpha_{m} H_{q}\left(\boldsymbol{e}\left(\boldsymbol{x}^{m}\right)\right), m=1, \cdots, N_{m c},
$$

where $q$ and $p$ are two parameters indicating the norms used for $\boldsymbol{e}$ and $\boldsymbol{u}$, respectively. A smaller value of $U\left(\boldsymbol{x}^{0}\right)$ indicates a higher yield. Following the suggestion in [25], we set $p=q=1$ and the weighting factor $\alpha_{m}=1$ in this thesis. These settings lead to an objective function in the following form [50]:

$$
\begin{aligned}
U\left(\boldsymbol{x}^{0}\right) & =\sum_{m \in M} \sum_{j \in J\left(\boldsymbol{x}^{m}\right)} e_{j}\left(\boldsymbol{x}^{m}\right) . \\
J\left(\boldsymbol{x}^{m}\right) & =\left\{j \mid e_{j}\left(\boldsymbol{x}^{m}\right)>0\right\} \\
M & =\left\{m \mid J\left(\boldsymbol{x}^{m}\right) \neq \emptyset\right\}
\end{aligned}
$$

In most practical cases, a reasonably large number of random comes are required to achieve an effective minimization of the objective function defined in (4.1)-(4.9).

\subsection{Proposed Parallel Space-Mapping Based Yield Optimization Technique Incorporating Trust Region Algorithm and PCE}

A direct application of the objective function $U\left(\boldsymbol{x}^{0}\right)$ is feasible if the responses are computed by circuit simulations. When the responses are obtained from EM 
simulations, it appears to be computationally expensive to apply $U\left(\boldsymbol{x}^{0}\right)$ to EM-based yield optimization directly [50]. To address this challenge, we propose in this chapter a parallel SM based yield-driven EM optimization technique incorporating trust region algorithm and $\mathrm{PCE}$. The proposed technique mainly consists of five parts, namely, fine model data generation with parallel computational method, surrogate modeling over multiple geometrical samples with parallel computation method, the

$l_{1}$ design centering algorithm to optimize the yield using the surrogate model, a novel trust region algorithm to update the trust region of the surrogate, and the PCE approach to yield verification on the fine model. We provide the details of these five parts in the subsequent sections.

\subsubsection{Fine Model Data Generation with Parallel Computa- tion Method}

Parallel computation is a powerful tool to accelerate the EM data generation process. Here, we propose to use parallel computational method to evaluate the fine model responses using multiple processors in parallel, thereby reducing the total CPU time of EM-based yield optimization. Let $N$ be the number of data points generated using the fine model. The fine model responses at $N$ data points are evaluated using $N$ parallel processors. Let $S_{p}$ and $\eta$ represent the parallel speedup factor and the parallel efficiency, respectively. The communication time between multiple processors running in parallel produces an overhead cost to the EM data generation process in each iteration. The speedup is defined as the ratio of the data generation 
time on a single processor over that on $N$ processors running in parallel [35], i.e.,

$$
S_{p}=\frac{\sum_{j=1}^{N} T_{j}}{T_{o}+\max _{1 \leq j \leq N} T_{j}},
$$

where $T_{o}$ represents the additional communication time for data generation using $N$ processors, and $T_{j}$ denotes the time for each fine model evaluation on the $j$ th processor. The parallel efficiency $\eta$ is defined as the ratio of the speedup factor over the total number of processors [35], i.e.,

$$
\eta=\frac{S_{p}}{N}
$$

Obviously, when all the $N$ processors have similar evaluation time $T_{j}$ and the overhead cost $T_{o}$ is much smaller than $T_{j}$, a large speedup and a high parallel efficiency can be achieved.

\subsubsection{Surrogate Modeling over Multiple Geometrical Sam- ples Using Parallel Computation Method}

Direct EM-based yield optimization with accurate full-wave EM simulations is computationally prohibitive. Therefore, the first step in the proposed technique is to develop a surrogate model to replace the fine model to achieve high-quality yield optimization solutions in an efficient manner. Same as all the SM-based approaches, we assume the availability of a computationally fast but not so accurate coarse model and an accurate but computationally expensive fine model. Let $\boldsymbol{R}^{c}(\boldsymbol{x})$ and $\boldsymbol{R}^{f}(\boldsymbol{x})$ denote the response vectors of the coarse and fine models corresponding to $\boldsymbol{x}$, respectively. We first establish a surrogate model combining the coarse model 
with a linear input mapping as follows:

$$
\begin{gathered}
\boldsymbol{x}_{c}=\boldsymbol{B} \boldsymbol{x}_{f}+\boldsymbol{c}, \\
\boldsymbol{R}^{s}\left(\boldsymbol{x}_{f}, \boldsymbol{w}\right)=\boldsymbol{R}^{c}\left(\boldsymbol{x}_{c}\right)=\boldsymbol{R}^{c}\left(\boldsymbol{B} \boldsymbol{x}_{f}+\boldsymbol{c}\right),
\end{gathered}
$$

where $\boldsymbol{x}_{c}$ and $\boldsymbol{x}_{f}$ are two vectors containing all the design variables of the coarse model and the fine model, respectively. $\boldsymbol{R}^{c}\left(\boldsymbol{x}_{c}\right)$ is the response vector of the coarse model corresponding to $\boldsymbol{x}_{c}$, while $\boldsymbol{R}^{s}\left(\boldsymbol{x}_{f}, \boldsymbol{w}\right)$ represents the response vector of the SM surrogate. $\boldsymbol{w}$ is a vector of mapping parameters which contain all the elements in $\boldsymbol{B}$ and $\boldsymbol{c}$, where $\boldsymbol{B}$ and $\boldsymbol{c}$ represent the coefficients in the linear mapping function.

The surrogate in the proposed technique is trained over multiple geometrical samples. The purpose is to make the surrogate valid in a relatively larger neighborhood in the design parameter space, thereby reducing the SM iterations in the whole optimization process. Let the nominal point at the $k$ th $(k=1,2, \ldots)$ iteration during yield optimization be denoted by $\boldsymbol{x}^{0, k}$, where $\boldsymbol{x}^{0,1}$ is initialized as the optimal solution from nominal optimization. In each iteration, a set of geometrical samples are generated in the neighborhood of $\boldsymbol{x}^{0, k}$ in the design parameter space. The fine model responses at these geometrical samples are to be used to train the surrogate model. In this work, star distribution is used as the sampling method to generate the geometrical samples around $\boldsymbol{x}^{0, k}$ in the design parameter space. Let $X_{T r}^{k}$ represent the set of geometrical samples in iteration $k$, then,

$$
X_{T r}^{k}=\left\{\boldsymbol{x}^{(1), k}, \boldsymbol{x}^{(2), k}, \cdots, \boldsymbol{x}^{(2 n+1), k}\right\} .
$$

Next, the fine model are evaluated at all these $2 n+1$ geometrical samples by using 
$2 n+1$ processors in parallel

$$
\begin{aligned}
\left\{\boldsymbol{R}^{f}\left(\boldsymbol{x}^{(i), k}\right) \mid i=1,2, \ldots, 2 n+1\right\} & \\
& =\left\{\boldsymbol{R}^{f}\left(\boldsymbol{x}^{(1), k}\right), \boldsymbol{R}^{f}\left(\boldsymbol{x}^{(2), k}\right), \ldots, \boldsymbol{R}^{f}\left(\boldsymbol{x}^{(2 n+1), k}\right)\right\} .
\end{aligned}
$$

In each iteration (say iteration $k$ ), the surrogate model is trained by using an optimization formulation with the objective of minimizing an error function. Let $E(\boldsymbol{w})$ be the error function. $E(\boldsymbol{w})$ is defined as the sum of the squared differences between the responses of the fine model and those of the surrogate at all the $2 n+1$ geometrical samples, i.e.,

$$
\begin{aligned}
E(\boldsymbol{w}) & =\sum_{l=1}^{2 n+1} e^{(l), k}(\boldsymbol{w}) \\
& =\sum_{l=1}^{2 n+1}\left\|\boldsymbol{R}^{f}\left(\boldsymbol{x}^{(l), k}\right)-\boldsymbol{R}^{s}\left(\boldsymbol{x}^{(l), k}, \boldsymbol{w}\right)\right\|^{2},
\end{aligned}
$$

where $\boldsymbol{R}^{f}\left(\boldsymbol{x}^{(l), k}\right)$ and $\boldsymbol{R}^{s}\left(\boldsymbol{x}^{(l), k}\right)$ denote the response vectors of the fine and coarse models corresponding to the $l$ th $(l=1,2, \ldots, 2 n+1)$ training sample $\boldsymbol{x}^{(l), k}$, respectively. The training process of the surrogate model can be denoted by

$$
\boldsymbol{w}_{k}=\arg \min _{w} E(\boldsymbol{w})
$$

where $\boldsymbol{w}_{k}$ contains the optimal parameters of the mapping function after training. Similar to the fine model data generation process, in this work, the training process of the surrogate model to match the fine model at $2 n+1$ data points also uses the parallel computation method [35]. This reduces the surrogate modeling time in each iteration, thereby further accelerating the overall yield optimization process. Once 
the training procedure is finished, a new set of mapping parameters is obtained. Next, we perform yield optimization using the updated surrogate to obtain the next optimal yield solution $\boldsymbol{x}^{0, k+1}$.

\subsubsection{The One-Sided $l_{1}$ Centering Algorithm for Yield Op- timization Using the Parallel SM Surrogate}

Once the development of the surrogate is done, we next perform yield optimization on the surrogate model with the same statistical distributions and design specifications as those in the original EM-based yield optimization problem. The yield optimization on the surrogate is effective provided that the surrogate model is well trained over a number of geometrical samples and that a suitable region of interest is defined for the surrogate.

In this work, we use the one-sided $l_{1}$ design centering algorithm [106] to optimize the yield of the surrogate. Let $\boldsymbol{e}^{s}(\boldsymbol{x})$ represent the error vector used to indicate how well the response vector of the surrogate model satisfies the design specifications. Similar to (4.1), we have

$$
\boldsymbol{e}^{s}(\boldsymbol{x})=\left[\begin{array}{lll}
e_{1}^{s} & e_{2}^{s} \cdots e_{N_{s}^{u}}^{s} \cdots e_{N_{s}}^{s}
\end{array}\right]^{T}
$$

For each random outcome $\boldsymbol{x}^{m}$, we have a corresponding error vector $\boldsymbol{e}^{s}\left(\boldsymbol{x}^{m}\right)$. The value of $e_{j}^{s}\left(\boldsymbol{x}^{m}\right)$, where $j=1, \ldots, N_{s}$, indicates the degree to which the surrogate model response violates the $j$ th design specification sample $S_{j}$. A larger value of $e_{j}^{s}\left(\boldsymbol{x}^{m}\right)$ implies that $S_{j}$ is violated to a larger degree.

To optimize the yield of the microwave structure at $\boldsymbol{x}^{0}$, the $l_{1}$ design centering 
algorithm minimizes the following objective function using a certain gradient-based optimization algorithm:

$$
\begin{aligned}
U^{s}\left(\boldsymbol{x}^{0}\right) & =\sum_{m \in M} \sum_{j \in J\left(\boldsymbol{x}^{m}\right)} e_{j}^{s}\left(\boldsymbol{x}^{m}\right), \\
J\left(\boldsymbol{x}^{m}\right) & =\left\{j \mid e_{j}^{s}\left(\boldsymbol{x}^{m}\right)>0\right\} \\
M & =\left\{m \mid J\left(\boldsymbol{x}^{m}\right) \neq \emptyset\right\}
\end{aligned}
$$

where $e_{j}^{s}\left(\boldsymbol{x}^{m}\right)$ is defined in the same way as that in (4.2), but this time the responses are evaluated from the surrogate model instead of the fine model, i.e.,

$$
e_{j}^{s}(\boldsymbol{x})= \begin{cases}R_{j}^{s}(\boldsymbol{x}, \boldsymbol{w})-S_{j}, & \text { if } 1 \leq j \leq N_{s}^{u}, \\ S_{j}-R_{j}^{s}(\boldsymbol{x}, \boldsymbol{w}), & \text { if } N_{s}^{u}<j \leq N_{s} .\end{cases}
$$

It is seen that the yield objective function defined in (4.19) is closely related to the number of failed designs. By minimizing (4.19), the yield of the fine model is expected to be increased. Therefore, the new nominal point used for yield verification on the fine model, $\boldsymbol{x}^{0, k+1}$, is obtained as follows:

$$
\boldsymbol{x}^{0, k+1}=\arg \min _{\boldsymbol{x}^{0}} U^{s}\left(\boldsymbol{x}^{0}\right)
$$

Note that by replacing the EM-based fine model with a computationally efficient surrogate model, and applying (4.19)-(4.21) on the surrogate, we avoid the large number of computationally expensive EM simulations and thus the EM-based yield optimization procedure can be greatly accelerated. 


\subsubsection{Proposed Trust Region Algorithm for Parallel SM Based Yield Optimization}

Since the surrogate model can only learn the EM behavior of the fine model in a certain region in the design parameter space, a trust region has to be defined for the surrogate in each iteration during yield optimization. The trust region is a region in the design parameter space beyond which the surrogate become unreliable and cannot represent the behavior of the fine model accurately. Therefore, the $l_{1}$ design centering algorithm should only explore the parameter space inside the trust region in order to have an effective minimization of the objective function defined in (4.19). Based on this idea, we propose a novel trust region algorithm to update the trust radius of each design parameter in each iteration in yield-driven design.

Let $\Omega^{k}$ be the trust region of the surrogate model in iteration $k$, defined as follows:

$$
\Omega^{k}=\left\{\boldsymbol{x} \mid x_{i}^{0, k}-\delta_{i}^{k} \leq x_{i} \leq x_{i}^{0, k}+\delta_{i}^{k}, \forall i=1, \ldots, n\right\}
$$

where $\delta_{i}^{k}$ represents the trust radius for the $i$ th design variable in iteration $k$. Let $\boldsymbol{\delta}^{k}=\left\{\delta_{1}^{k}, \delta_{2}^{k}, \ldots, \delta_{n}^{k}\right\}$ be the set containing the trust radii for all the design variables. The update of the trust radius for each design parameter depends on the ratio of yield improvements of the surrogate model over that of the fine model between two consecutive iterations. Specifically, between iteration $k$ and iteration $k+1$, we evaluate the yield values of the fine model (denoted by $Y_{f}\left(\boldsymbol{x}^{0, k}\right)$ and $Y_{f}\left(\boldsymbol{x}^{0, k+1}\right)$ ) and the yield value of the surrogate model (denoted by $Y_{s}\left(\boldsymbol{x}^{0, k+1}\right)$ ) via the PCE approach. Define a parameter $r$ to be the ratio of yield increase on the surrogate 
model over that on the fine model as follows:

$$
r= \begin{cases}\frac{Y_{f}\left(\boldsymbol{x}^{0, k+1}\right)-Y_{f}\left(\boldsymbol{x}^{0, k}\right)}{Y_{s}\left(\boldsymbol{x}^{0, k+1}\right)-Y_{f}\left(\boldsymbol{x}^{0, k}\right)}, & \text { if } Y_{f}\left(\boldsymbol{x}^{0, k+1}\right) \geq Y_{f}\left(\boldsymbol{x}^{0, k}\right), \\ -1, & \text { otherwise. }\end{cases}
$$

The parameter $r$ can be used as an indicator to update the trust radius of each design parameter due to the following reasons: 1) when $Y_{f}\left(\boldsymbol{x}^{0, k+1}\right)<Y_{f}\left(\boldsymbol{x}^{0, k}\right)$, this means that yield optimization on the surrogate is not effective, i.e., it cannot increase the yield of the fine model. Therefore, the trust radius of each design parameter should shrink; 2) when $Y_{f}\left(\boldsymbol{x}^{0, k+1}\right) \geq Y_{f}\left(\boldsymbol{x}^{0, k}\right)$, this implies that the yield of the fine model is increased after performing yield optimization on the surrogate. Depending on how large the yield improvement on the fine model is, one can choose to keep, enlarge, or shrink the current trust radius. In this thesis, we use the following formulas to update the trust radii of the design parameters in the $k$ th iteration [109]:

$$
\boldsymbol{\delta}^{k+1}= \begin{cases}0.69 \boldsymbol{\delta}^{k}, & \text { if } r<0.1 \\ \min \left\{1.3 \boldsymbol{\delta}^{k}, \boldsymbol{\Delta}_{\max }\right\}, & \text { if } r>0.75 \\ \boldsymbol{\delta}^{k}, & \text { otherwise }\end{cases}
$$

where $\boldsymbol{\Delta}_{\max }$ denotes the maximal allowed trust radii for the design variables. In this thesis, $\boldsymbol{\Delta}_{\max }$ is set as $50 \%$ of the initial nominal values of the design variables. 


\subsubsection{Incorporating the PCE Approach for Yield Verifica- tion on the Fine Model}

In each iteration, after the yield optimization on the surrogate is done and a new nominal point is found, the yield value on the fine model at the new nominal point has to be verified. To further speed up the overall optimization process, we propose to use the PCE approach to verify the yield of the fine model in each iteration during yield optimization. To realize PCE-based yield estimation, one has to first transform the original random parameters $\boldsymbol{x}$ to independent standard random parameters $\boldsymbol{\xi}$. Next, the stochastic expansion has to be applied in the " $\boldsymbol{\xi}$-space" [93] to express the function between the stochastic quantity and $\boldsymbol{\xi}$. In the $k$ th iteration, let $R_{j}^{f}\left(\boldsymbol{x}^{0, k}, \boldsymbol{\xi}\right)$ be the response of the fine model at the frequency point where the $j$ th design

specification sample exists. Via PCE, the relationship between $R_{j}^{f}\left(\boldsymbol{x}^{0, k}, \boldsymbol{\xi}\right)$ and $\boldsymbol{\xi}$ is represented by the weighted sum of a set of orthogonal basis functions as follows:

$$
R_{j}^{f}\left(\boldsymbol{x}^{0, k}, \boldsymbol{\xi}\right)=\sum_{i=0}^{P} a_{i j}\left(\boldsymbol{x}^{0, k}\right) \Phi_{i}(\boldsymbol{\xi}),
$$

where $\Phi_{i}(\cdot)$ represents the generalized basis function in PCE. The total number of terms in $(4.25)$ is $P+1 . a_{i j}$ represents the PCE coefficients, which are also the weighting coefficients for different basis functions at different EM responses.

Given that the basis functions $\Phi_{i}(\boldsymbol{\xi})$ are orthogonal to each other, the coefficients of PCE, $a_{i j}$, are evaluated as follows:

$$
a_{i j}\left(\boldsymbol{x}^{0, k}\right)=\frac{\int_{\Omega^{n}} R_{j}^{f}\left(\boldsymbol{x}^{0, k}, \boldsymbol{\xi}\right) \Phi_{i}(\boldsymbol{\xi}) \rho(\boldsymbol{\xi}) d \boldsymbol{\xi}}{\int_{\Omega^{n}} \Phi_{i}^{2}(\boldsymbol{\xi}) \rho(\boldsymbol{\xi}) d \boldsymbol{\xi}},
$$

where $\Omega^{n}$ represents the $n$-dimensional random space of $\boldsymbol{\xi} \cdot \rho(\boldsymbol{\xi})$ represents the joint 


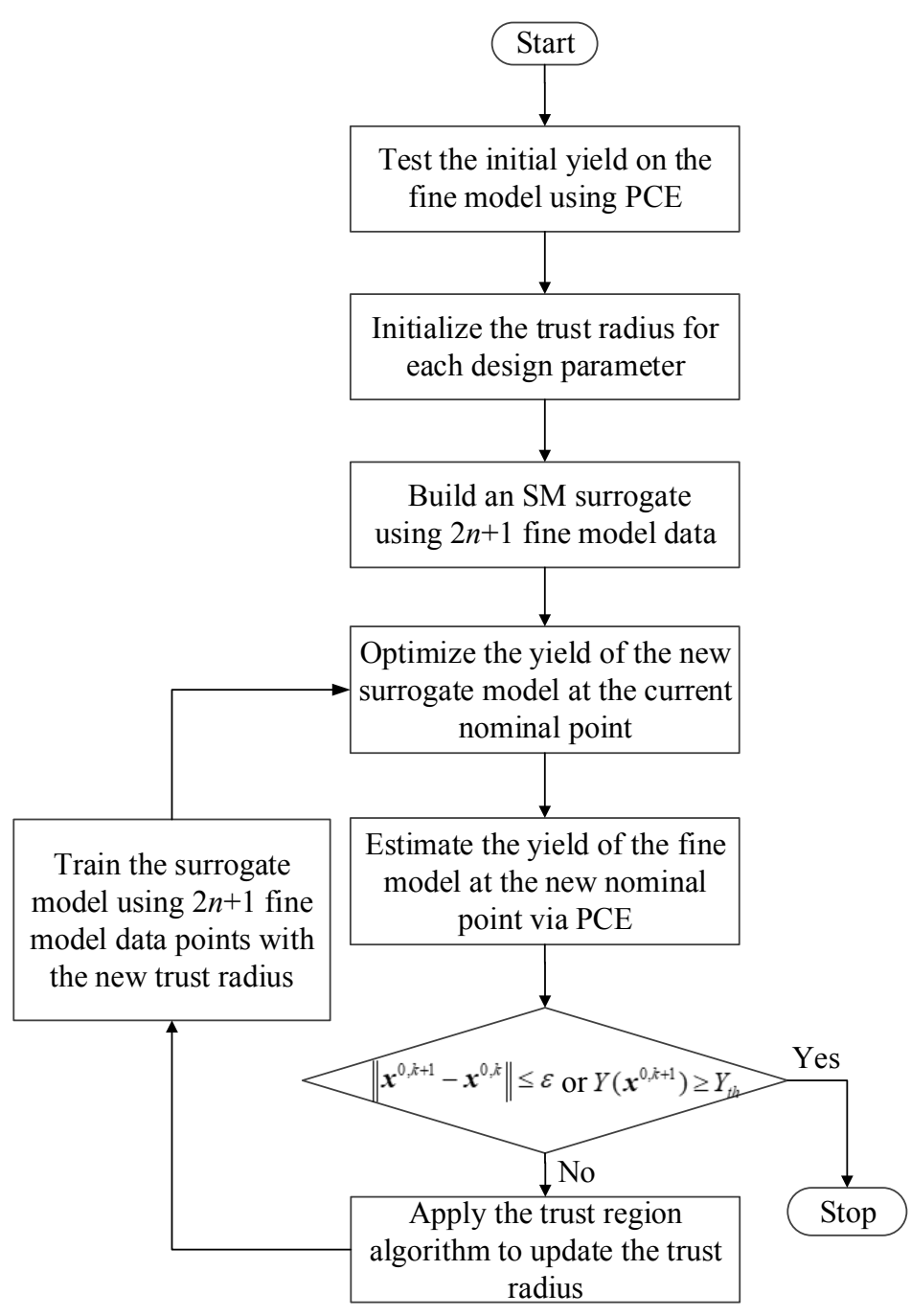

Figure 4.1: Flow chart of the proposed yield optimization technique.

probability density function (PDF) of the transformed parameters $\boldsymbol{\xi}$. Numerical quadrature based on sparse grid techniques [95] are typically used to evaluate the multi-dimensional integrations in (4.26). Let $N_{s g}$ be the number of samples in sparse grid techniques.

To perform yield estimation of the fine model, a number of PCE models have 
to be constructed at all the frequencies of interest. More specifically, a single PCE model has to be constructed for each frequency point under consideration. In other words, the total number of PCE models is equal to the total number of frequency points in the whole frequency range of interest. Then, an MC analysis needs to be performed on the PCE models taking into consideration all the design specifications [95]. We summarize the yield verification on the fine model via PCE at iteration $k$ as follows:

Step 1) Generate $N_{s g}$ samples of the transformed parameters $\boldsymbol{\xi}$, i.e., $\left\{\boldsymbol{\xi}^{(1)}, \boldsymbol{\xi}^{(2)}, \cdots\right.$, $\left.\boldsymbol{\xi}^{\left(N_{s g}\right)}\right\}$, in the " $\boldsymbol{\xi}$-space" following the rules of the sparse grid technique.

Step 2) Transform the $N_{s g}$ samples in the " $\boldsymbol{\xi}$-space" back into the " $\boldsymbol{x}$-space", to obtain a set of geometrical parameter samples around the nominal point $\boldsymbol{x}^{0, k}$.

Step 3) Evaluate the responses of the fine model at the $N_{s g}$ geometrical parameter samples in parallel.

Step 4) Numerically evaluate the PC coefficients $a_{i j}$ using numerical quadrature based on the sparse grid technique [50].

Step 5) Perform an MC analysis on the PCE models taking into consideration all the design specifications to obtain the yield verification result on the fine model at $\boldsymbol{x}^{0, k}$.

As demonstrated in [39], the PCE approach to EM-based yield estimation is much more computationally efficient than traditional MC analysis, since an accurate 
evaluation of PCE coefficients typically requires much fewer EM samples than the MC analysis does.

\subsubsection{The Proposed Yield Optimization Algorithm}

The overall yield optimization procedure terminates if the difference of $\boldsymbol{x}^{0, k}$ between subsequent iterations is sufficiently small or the yield value of the fine model at the new nominal point is higher than a user-defined threshold, i.e.,

$$
\begin{aligned}
& \left\|\boldsymbol{x}^{0, k+1}-\boldsymbol{x}^{0, k}\right\| \leq \varepsilon, \\
& \text { or } \quad Y\left(\boldsymbol{x}^{0, k+1}\right) \geq Y_{t h},
\end{aligned}
$$

where $\varepsilon$ and $Y_{t h}$ are both user-defined criteria. The flow chart of the proposed yield optimization algorithm is shown in Fig. 4.1, which can also be summarized as follows:

Step 1) Set iteration counter $k=1$. Initialize the termination criteria $\varepsilon$ and $Y_{\text {th }}$ (the desired yield value). Initialize the trust radius for each design parameter. Set the perturbation sizes in star distribution for the design parameters the same as their trust radius.

Step 2) Initialize the nominal point $\boldsymbol{x}^{0, k}$ to be the optimal solution from nominal optimization $\boldsymbol{x}_{i n i}^{0}$. Estimate the yield of the fine model at $\boldsymbol{x}^{0, k}$ using the PCE approach described in Section 4.3.5.

Step 3) If $k \geq 2$, update the trust radius for each design parameter using (4.23) and (4.24), and update the perturbation size to be the same as the updated 
trust radius.

Step 4) Generate $2 n+1$ geometrical samples, $X_{T r}^{k}$, following star distribution based on the updated trust region for training the surrogate.

Step 5) Build a new surrogate model $\boldsymbol{R}^{s}(\boldsymbol{x}, \boldsymbol{w})$ using (4.14)-(4.17) and obtain the optimal mapping parameters $\boldsymbol{B}$ and $\boldsymbol{c}$.

Step 6) Perform yield optimization on the new surrogate by solving (4.21) using a gradient-based algorithm, e.g., the quasi-Newton method, to find the next optimal yield solution $\boldsymbol{x}^{0, k+1}$.

Step 7) Estimate the yield of the fine model at the new nominal point $\boldsymbol{x}^{0, k+1}$ using the PCE approach.

Step 8) If $\left\|\boldsymbol{x}^{0, k+1}-\boldsymbol{x}^{0, k}\right\| \leq \varepsilon$ or $Y\left(\boldsymbol{x}_{0, k+1}\right) \geq Y_{t h}$ is satisfied, go to Step 9$)$, otherwise, set $k=k+1$ and go to Step 3).

Step 9) Output the final optimal yield solution $\boldsymbol{x}^{*}=\boldsymbol{x}^{0, k}$ and the final yield value $Y^{*}=Y_{f}\left(\boldsymbol{x}^{0, k}\right)$.

\subsubsection{Discussion}

In the proposed technique, we choose to use the PCE approach to verify the yield of the fine model during the overall yield optimization process. The PCE approach serves as an efficient vehicle to further speed up the proposed technique. There also exist some other methods in the literature that can avoid the high computational costs in traditional Monte Carlo based analysis, such as the stochastic testing 
method [110], the polynomial chaos-Kriging (PC-Kriging) method [111], and the neural network based technique [112]. They all perform well for statistical analysis and/or yield estimation in different applications. Considering that the main focus of this study is yield-driven EM optimization, a comprehensive comparison between these methods and the PCE approach is beyond the scope of this study. However, upon being used properly, other efficient methods such as those in [110]-[112] can also be incorporated in the proposed technique to achieve efficient yield optimization of microwave structures.

\subsection{Application Examples}

\subsubsection{Yield Optimization of a Low-Pass Elliptic Microstrip Filter}

In this example, we aim to perform yield optimization for a two-section low-pass elliptic microstrip filter [35], as shown in Fig. 4.2. We consider six design variables, i.e., $\boldsymbol{x}=\left[\begin{array}{llllll}L_{1} & L_{2} & L_{c 1} & L_{c 2} & W_{c} & G_{c}\end{array}\right]^{T}$ (mil), and assume that the variables have independent normal distributions to allow yield optimization. The initial nominal point obtained from nominal EM optimization is $\boldsymbol{x}_{\text {ini }}^{0}=\left[\begin{array}{lll}44.79825 & 171.68189 & 165.44252\end{array}\right.$ $45.058736 .061973 .36753]^{T}$ (mil). The standard deviation of each design variable is $2 \%$ of its mean value. The design specifications for the filter are defined as

$$
\begin{aligned}
& \left|S_{21}\right| \geq-0.92 \mathrm{~dB}, \quad \text { for } 1.0 \mathrm{GHz} \leq \omega \leq 1.97 \mathrm{GHz} \\
& \left|S_{21}\right| \leq-18 \mathrm{~dB}, \quad \text { for } 2.3 \mathrm{GHz} \leq \omega \leq 4.0 \mathrm{GHz}
\end{aligned}
$$

The HFSS EM simulator with a fast simulation feature is used to perform fine 


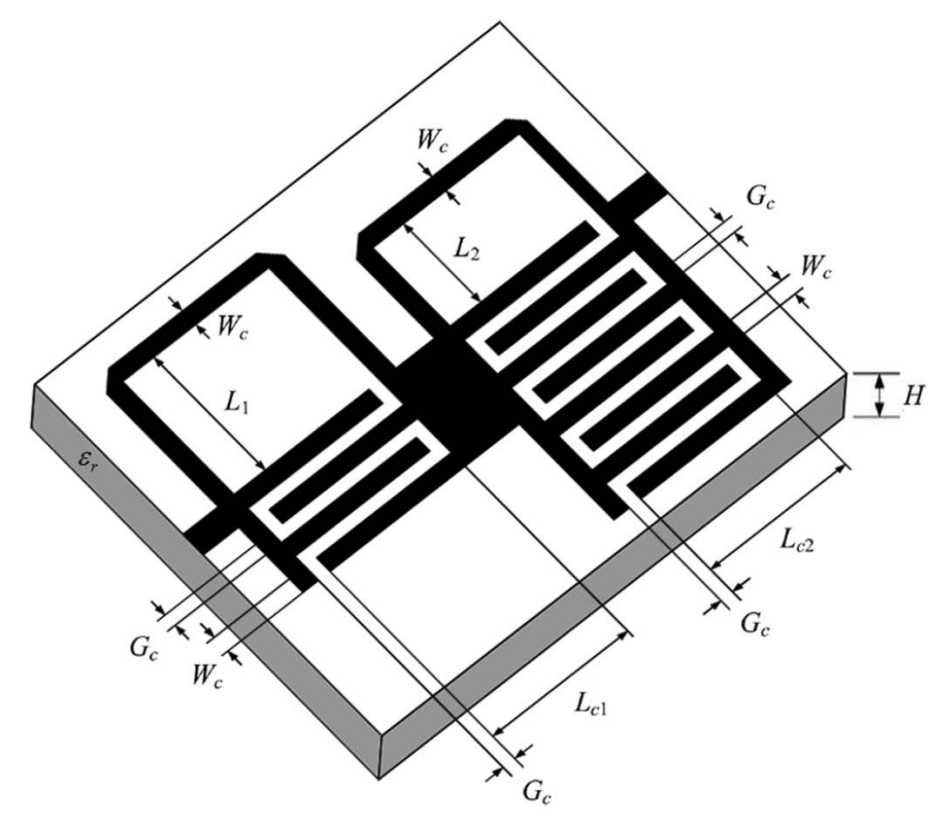

Figure 4.2: The structure of the low-pass elliptic microstrip filter used in EM simulation and yield optimization, from [35].

model evaluations. The coarse model is the equivalent circuit for the low-pass filter using simple transmission lines [35]. NeuroModelerPlus software is used to program the coarse model, implement surrogate model training over multiple geometrical samples, and perform yield optimization on the surrogate model. The initial yield value of the fine model at the initial nominal point is $56 \%$. The initial trust radius of each design parameter is set as $5 \%$ of its nominal value, i.e., $\boldsymbol{\delta}^{1}=0.05 \times \boldsymbol{x}_{i n i}^{0}$. The stop criteria of the propose yield optimization algorithm are set as $\varepsilon=1.0 e-5$ and $Y_{t h}=75 \%$. A cluster of computers is used to allow parallel processing for parallel data generation.

The proposed yield optimization technique stops after two SM iterations, meeting the user-defined yield criterion $Y_{t h}$. The final optimal yield solution is $\boldsymbol{x}^{*}=$ 
[43.91140 158.20101 163.80511 46.465446 .235983 .22687$]^{T}$ (mil) with a yield value of $77 \%$ of the fine model. The yield value of the fine model during yield optimization is verified by the PCE approach. For a further verification, we evaluate the responses of the fine model at a sufficiently large number $\left(N_{m c}\right)$ of random outcomes around the initial and final design solutions, and obtain the corresponding yield values using Monte Carlo analysis, as shown in Fig. 4.3. To obtain the reasonable value of $N_{m c}$ in Monte Carlo analysis, the yield at the initial nominal point is estimated with different number of random samples. We gradually increase the value of $N_{m c}$ until a convergence on the yield value is observed (which happens when $N_{m c}=100$ ). The same value is used for the number of random outcomes during yield optimization on the surrogate model. It is observed from Fig. 4.3 that a significant increase on the yield value is obtained, which demonstrates the effectiveness of the proposed technique.

For this example, we have $2 n+1=13$ data samples, so 13 processors are used to perform fine model evaluations in parallel. The time for fine model evaluations using the parallel computation method is $32 \mathrm{~min}$, while that using the sequential computation method is $372 \mathrm{~min}$. This results in a speedup of 11.6 and a parallel efficiency $(\eta)$ of about $89.5 \%$. This speedup contributes to the total CPU time reduction of the proposed yield optimization technique. For the purpose of comparison, we also apply the standard SM approach to optimize the yield for this example. For the standard SM, the surrogate model is built at only one geometrical sample in each SM iteration without using the parallel computation method. Specifically, in each iteration, the response of the fine model at the current nominal point is used to train 
Table 4.1: Comparison of Standard SM based Yield Optimization Technique and the Proposed Yield Optimization Technique for the Lowpass Microstrip Filter

\begin{tabular}{l|l|l}
\hline \hline $\begin{array}{l}\text { Yield optimization } \\
\text { technique }\end{array}$ & $\begin{array}{l}\text { Standard SM } \\
\text { based yield } \\
\text { optimization } \\
\text { technique }\end{array}$ & $\begin{array}{l}\text { Proposed yield } \\
\text { optimization } \\
\text { technique }\end{array}$ \\
\hline Initial yield of fine model & $56 \%$ & $56 \%$ \\
\hline Number of SM iterations & 4 & 2 \\
\hline $\begin{array}{l}\text { Fine model evaluation } \\
\text { time }\end{array}$ & $29 \mathrm{~min} \times 4$ & $32 \mathrm{~min} \times 2$ \\
\hline $\begin{array}{l}\text { Surrogate model training } \\
\text { time }\end{array}$ & $12 \mathrm{~s} \times 4$ & $40 \mathrm{~s} \times 2$ \\
\hline $\begin{array}{l}\text { Total surrogate yield op- } \\
\text { timization time }\end{array}$ & $24 \mathrm{~min}$ & $11 \mathrm{~min}$ \\
\hline Total CPU time & $141 \mathrm{~min}$ & $76 \mathrm{~min}$ \\
\hline Final yield of fine model & $62 \%$ & $77 \%$ \\
\hline \hline
\end{tabular}

the surrogate model. The comparison between the standard SM based yield optimization technique and the proposed yield optimization technique in terms of the yield improvement and total CPU time is shown in Table 4.1. Note that the CPU time for yield verification on the fine model in each iteration is omitted for both methods. As can be seen in the table, the standard SM based yield optimization terminates after four iterations, achieving a small yield increase of the fine model. The reason behind this is that standard SM builds the surrogate model over a single point. Hence, the surrogate model is valid in a smaller region than that built with the parallel SM approach, making it difficult to achieve an effective yield optimization of the surrogate. It is also seen from the table that, compared with the standard 


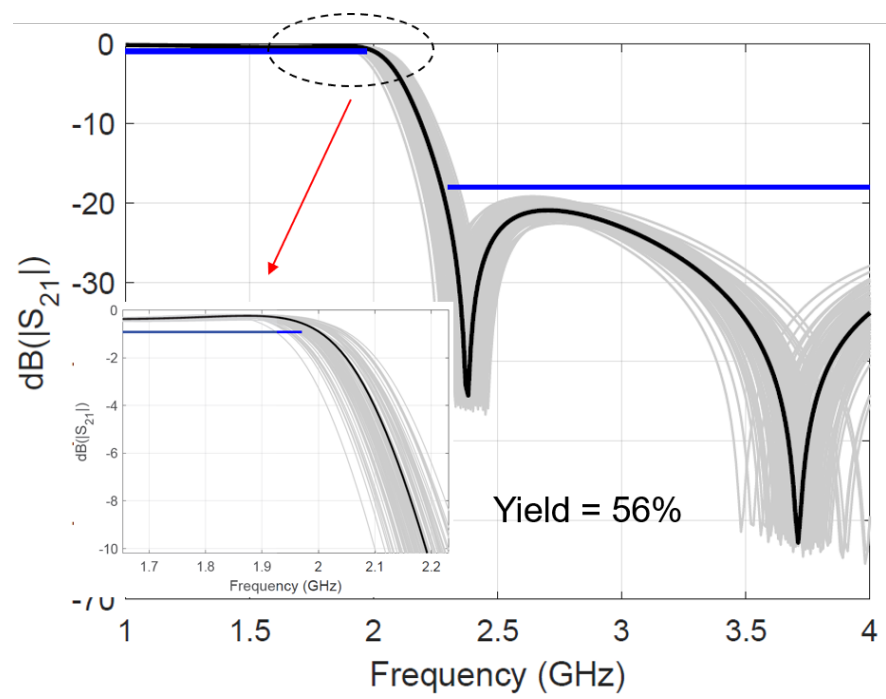

(a)

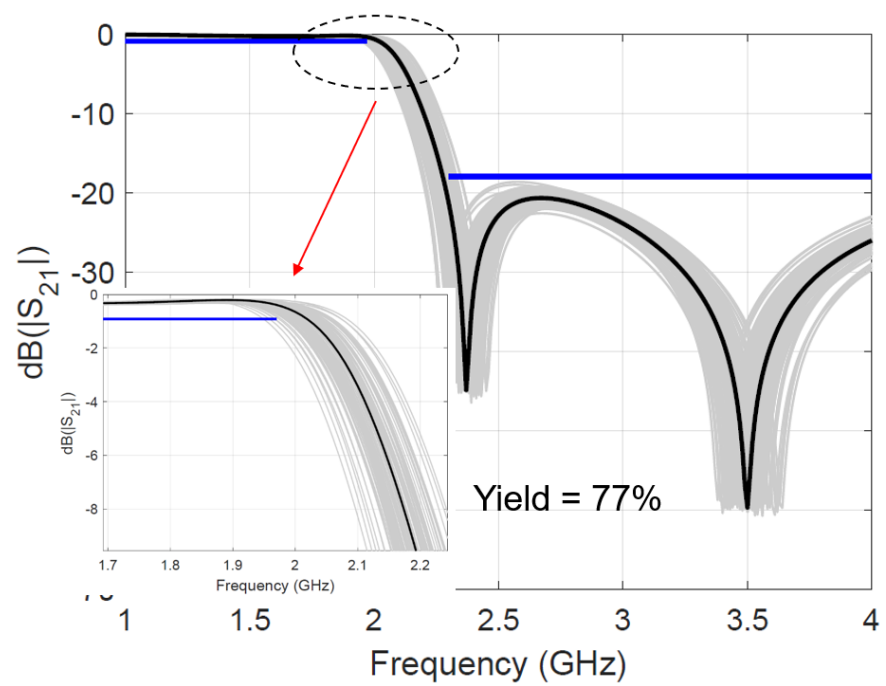

(b)

Figure 4.3: Yield optimization results of the low-pass microstrip filter using the proposed technique: (a) Before and (b) After yield optimization. In both figures, the grey dashed lines indicate 100 random samples in Monte Carlo analysis, while the black solid line indicates the nominal response. 
SM based yield optimization technique, the proposed yield optimization technique achieves a much greater yield increase in shorter CPU time. This is because our proposed technique builds the surrogate model over multiple geometrical samples, which enables the surrogate to be valid in a larger neighborhood. Therefore, the proposed technique is able to find the optimal yield solution in fewer SM iterations, accelerating the overall yield optimization process.

\subsubsection{Yield Optimization of a Bandstop Microstrip Filter with Open Stubs}

In the second example, we perform yield optimization for a bandstop microstrip filter with quarter-wave resonant open stubs [35], as shown in Fig. 4.4. The filter has five design variables, i.e., $\boldsymbol{x}=\left[\begin{array}{lllll}W_{1} & W_{2} & L_{0} & L_{1} & L_{2}\end{array}\right]^{T}$ (mil). We assume independent normal distributions for the design variables to realize yield estimation and optimization, with the standard deviation of each design variable being $0.5 \%$ of its mean value. The initial nominal point obtained from nominal EM optimization is $\boldsymbol{x}_{i n i}^{0}=\left[\begin{array}{lllll}5.83198 & 14.68157 & 120.06024 & 119.10691 & 110.10827\end{array}\right]^{T}$ (mil). The design specifications for the filter are defined as

$$
\begin{array}{ll}
\left|S_{21}\right| \geq 0.9, & \text { for } 5.0 \mathrm{GHz} \leq \omega \leq 8.0 \mathrm{GHz} \\
\left|S_{21}\right| \leq 0.05, & \text { for } 9.3 \mathrm{GHz} \leq \omega \leq 10.7 \mathrm{GHz} \\
\left|S_{21}\right| \geq 0.9, & \text { for } 12.0 \mathrm{GHz} \leq \omega \leq 15.0 \mathrm{GHz}
\end{array}
$$

The HFSS EM simulator with a fast simulation feature is used to perform fine model evaluations. The coarse model is the equivalent circuit for the bandstop filter using simple transmission lines based on $A D S$ [35]. NeuroModelerPlus software is 


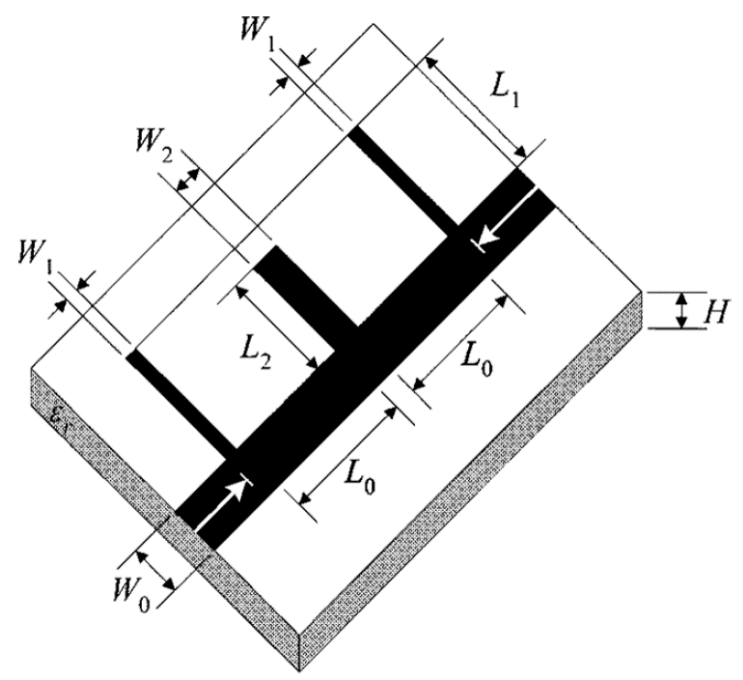

Figure 4.4: The structure of the bandstop microstrip filter defined for EM simulation and yield optimization, from [35].

used to program the coarse model, implement surrogate model training, and perform the yield optimization on the surrogate model. The yield value of the fine model at the initial nominal point is $22 \%$. The initial trust radius of each design parameter is set as $5 \%$ of its nominal value, i.e., $\boldsymbol{\delta}^{1}=0.05 \times \boldsymbol{x}_{i n i}^{0}$. The stop criteria of the propose yield optimization algorithm is set as $\varepsilon=1.0 e-5$ and $Y_{t h}=80 \%$. The proposed yield optimization technique terminates after two SM iterations, satisfying the user-defined stopping criterion $\left\|\boldsymbol{x}^{0, k+1}-\boldsymbol{x}^{0, k}\right\| \leq 1.0 e-5$. The final optimal yield solution is $\boldsymbol{x}^{*}=\left[\begin{array}{lllll}5.86118 & 14.59794 & 117.55622 & 121.90948 & 107.62781\end{array}\right]^{T}(\mathrm{mil})$ with a yield value of $46 \%$ of the fine model. The yield values at the initial nominal solution and the final optimal yield solution are both further verified by the Monte Carlo-based yield estimation, as shown in Fig. 4.5.

For this example, we have $2 n+1=11$ data samples, so 11 processors are used 
Table 4.2: Comparison of Standard SM based Yield Optimization Technique and the Proposed Yield Optimization Technique for the Bandstop Microstrip Filter

\begin{tabular}{l|l|l}
\hline \hline $\begin{array}{l}\text { Yield optimization } \\
\text { algorithm }\end{array}$ & $\begin{array}{l}\text { Standard SM } \\
\text { approach to } \\
\text { yield optimiza- } \\
\text { tion }\end{array}$ & $\begin{array}{l}\text { Proposed yield } \\
\text { optimization } \\
\text { technique }\end{array}$ \\
\hline Initial yield of fine model & $22 \%$ & $22 \%$ \\
\hline Number of iterations & 5 & 2 \\
\hline $\begin{array}{l}\text { Fine model evaluation } \\
\text { time }\end{array}$ & $14 \mathrm{~min} \times 5$ & $16 \mathrm{~min} \times 2$ \\
\hline $\begin{array}{l}\text { Surrogate model training } \\
\text { time }\end{array}$ & $20 \mathrm{~s} \times 5$ & $3 \mathrm{~min} \times 2$ \\
\hline $\begin{array}{l}\text { Total surrogate yield op- } \\
\text { timization time }\end{array}$ & $22 \mathrm{~min}$ & $15 \mathrm{~min}$ \\
\hline Total CPU time & $94 \mathrm{~min}$ & $53 \mathrm{~min}$ \\
\hline Final Yield of fine model & $39 \%$ & $46 \%$ \\
\hline \hline
\end{tabular}

to perform fine model evaluations in parallel. The time for fine model evaluations using the parallel computation method is $16 \mathrm{~min}$, while that using the sequential computation method is 157 min. This results in a speedup of 9.8 and a parallel efficiency $(\eta)$ of about $89.2 \%$. This speedup contributes to the total CPU time reduction of the proposed yield optimization technique. For comparison purposes, the standard SM approach is also applied to optimize the yield for this example. In each iteration during yield optimization, the surrogate model is trained with the EM response at the current nominal point. Table 4.2 compares the standard SM based yield optimization technique and the proposed technique in terms of the yield improvement and the total CPU time. It is observed from the table 
that, the proposed yield optimization technique achieves a higher yield increase using fewer SM iterations compared with the standard SM based yield optimization technique. Specifically, the standard SM optimization terminates after five SM iterations with a yield value of $39 \%$ of the fine model, while the proposed technique terminates after two SM iterations with a yield value of $46 \%$ of the fine model. This is because the surrogate model in the proposed technique is trained to be valid in larger neighborhood than that in the standard SM. Therefore, the trust region defined for the surrogate in the proposed technique can be larger than that in the standard SM, which increases the ability of improving the yield of the fine model for the proposed technique. These advantages ultimately allow the proposed technique to achieve a greater yield increase of the microwave structure in shorter CPU time compared with the standard SM based yield optimization technique. 


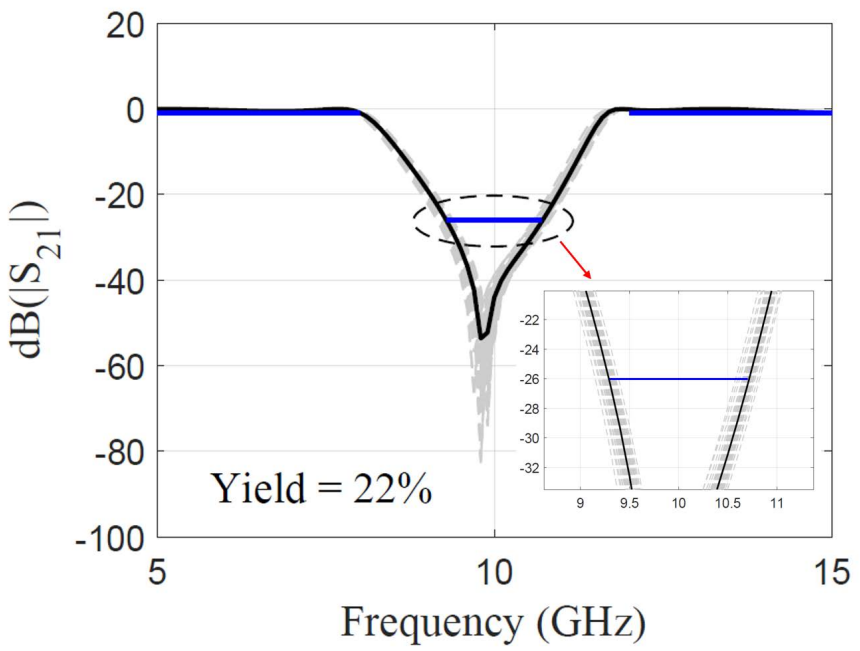

(a)

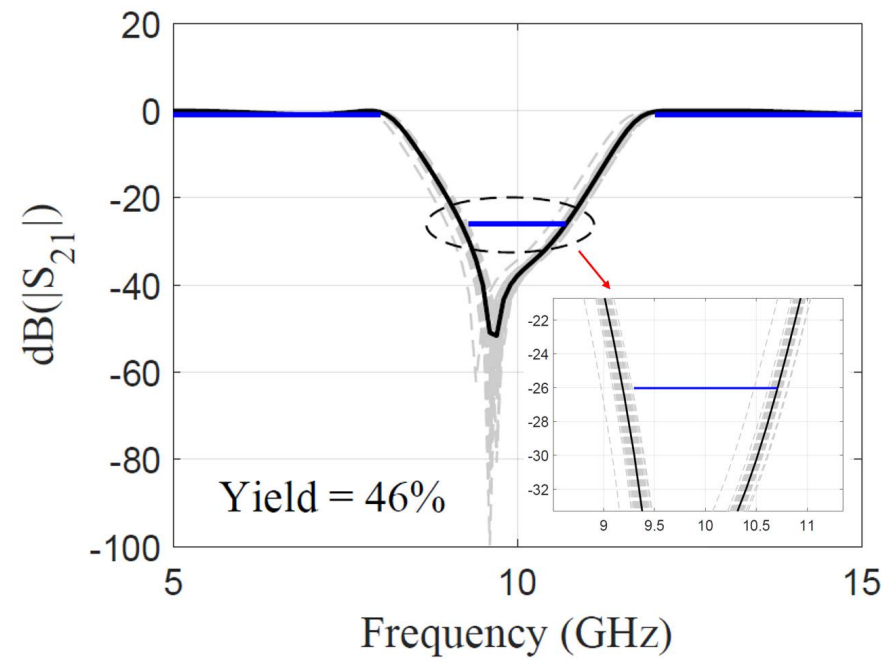

(b)

Figure 4.5: Yield optimization results of the bandstop microstrip filter using the proposed technique: (a) Before and (b) After yield optimization. In both figures, the grey dashed lines indicate 100 random samples in Monte Carlo analysis, while the black solid line indicates the nominal response. 


\section{Chapter 5}

\section{Non-Surrogate-Based Approach to Yield-Driven EM Optimization Exploiting PCE}

\section{$5.1 \quad$ Introduction}

The parallel SM based technique presented in the previous chapter is able to accelerate the overall yield optimization process by reducing the number of SM iterations. However, it requires the availability of an equivalent circuit coarse model. In many practical cases, equivalent circuit coarse models are not always available [91]. To address this situation, in this chapter, we propose a novel non-surrogate-based approach to yield-driven EM optimization exploiting PCE. For the first time, the use of PCE approach is elevated from EM-based yield estimation to EM-based yield optimization. The proposed approach provides systematic formulation and sensitivity formulas of EM-based yield optimization and does not require the availability of a coarse model. In the proposed approach, we first formulate a novel objective function for yield-driven EM optimization. By incorporating PCE coefficients into 
the formulation, the objective function is analytically related to yield optimization variables which are the nominal point. We then derive the sensitivity formulas of the PCE coefficients w.r.t. the nominal point. The sensitivities of the objective function w.r.t. the nominal point are derived based on the sensitivity formulas of the PCE coefficients. These sensitivities are then used in gradient-based optimization algorithms to find the optimal yield solution iteratively. The proposed objective function requires fewer EM simulations to provide reliable yield representation than that in the conventional Monte Carlo-based yield optimization approach. As a result, the number of EM simulations required to find the update direction and proper step size for the change of the nominal point is reduced at each iteration of optimization. This allows the proposed approach to achieve similar yield increase using much fewer EM simulations or greater yield increase using similar number of EM simulations compared to the conventional yield optimization approach. Three waveguide filter examples are used to demonstrate the advantages of our proposed approach.

\subsection{Formulations of the PCE Approach}

In this section, we give a brief overview of formulations of the PCE approach and establish necessary notations for the descriptions of the proposed approach.

Let $\boldsymbol{x}$ represent the vector of $n$ design parameters (e.g., geometrical parameters) of the EM structure, where $\boldsymbol{x}=\left[x_{1}, x_{2}, \cdots, x_{n}\right]^{T}$. Let $\boldsymbol{x}^{0}$ be the nominal point of $\boldsymbol{x}$. For statistical analysis of EM structures, the actual values of design parameters of the manufactured devices are spread around $\boldsymbol{x}^{0}$ following certain (uniform, 
normal, etc.) distributions. A key component of PCE-based statistical analysis is performing a transformation of parameters from the original random parameters $\boldsymbol{x}$ to independent standard random parameters $\boldsymbol{\xi}$ and then applying the stochastic expansion in the transformed space (i.e., the " $\boldsymbol{\xi}$-space") $[93]$. Let $\boldsymbol{\xi}=\left[\xi_{1}, \cdots, \xi_{n}\right]^{T}$ be the vector of the independent standard random parameters. For example, $\xi_{1}, \cdots, \xi_{n}$ are with zero mean and unit variance if $x_{1}, x_{2}, \cdots, x_{n}$ have normal distributions, or with support $[-1,1]$ if $x_{1}, x_{2}, \cdots, x_{n}$ are uniformly distributed. In the context of EM-based yield optimization, the change of $\boldsymbol{x}^{0}$ should be considered when performing the transformation as the nominal point is a variable. We thus denote this transformation as $\boldsymbol{\xi}=T\left(\boldsymbol{x}^{0}, \boldsymbol{x}\right)$ with the inverse transformation denoted as

$$
\boldsymbol{x}=T^{-1}\left(\boldsymbol{x}^{0}, \boldsymbol{\xi}\right) .
$$

The design goals are typically defined by a set of specifications imposed on the response of the EM structure at each frequency of interest. Let $S_{j}$ be the $j$-th design specification sample, where $j=1, \cdots, m$. The symbol $m$ denotes the number of specification samples, including samples for upper specifications and samples for lower specifications. Let $m_{u}$ be the number of upper specification samples. For convenience of description, we assume that $S_{1}, \cdots, S_{m_{u}}$ are upper specification samples and that $S_{m_{u}+1}, \cdots, S_{m}$ are lower specification samples. Let $R_{j}(\boldsymbol{x})$ be the EM response at the frequency of interest that corresponds to $S_{j}$. As we focus on yield optimization where the nominal point $\boldsymbol{x}^{0}$ is a variable, we intend to write $R_{j}(\boldsymbol{x})$ in a form where $\boldsymbol{x}^{0}$ is explicitly presented. Substituting the reverse transformation (5.1) into $R_{j}(\boldsymbol{x})$ allows us to have $R_{j}(\boldsymbol{x})=R_{j}\left(T^{-1}\left(\boldsymbol{x}^{0}, \boldsymbol{\xi}\right)\right)$. An error vector $\boldsymbol{e}(\boldsymbol{x})$ 
can be defined to measure the degree to which the response satisfies the design specifications as follows

$$
\boldsymbol{e}(\boldsymbol{x})=\boldsymbol{e}\left(T^{-1}\left(\boldsymbol{x}^{0}, \boldsymbol{\xi}\right)\right)=\left[e_{1} e_{2} \cdots e_{m_{u}} \cdots e_{m}\right]^{T},
$$

where the $j$-th element in the error vector, $e_{j}\left(T^{-1}\left(\boldsymbol{x}^{0}, \boldsymbol{\xi}\right)\right)$, is given by

$$
e_{j}\left(T^{-1}\left(\boldsymbol{x}^{0}, \boldsymbol{\xi}\right)\right)= \begin{cases}R_{j}\left(T^{-1}\left(\boldsymbol{x}^{0}, \boldsymbol{\xi}\right)\right)-S_{j}, & \text { if } 1 \leq j \leq m_{u}, \\ S_{j}-R_{j}\left(T^{-1}\left(\boldsymbol{x}^{0}, \boldsymbol{\xi}\right)\right), & \text { if } m_{u}<j \leq m .\end{cases}
$$

In practice, it is possible to impose both an upper specification and a lower specification on the response at a common frequency of interest. For example, if $S_{j_{1}}$ and $S_{j_{2}}$ are the upper specification and the lower specification at a common frequency, respectively, then the responses $R_{j_{1}}\left(T^{-1}\left(\boldsymbol{x}^{0}, \boldsymbol{\xi}\right)\right)$ and $R_{j_{2}}\left(T^{-1}\left(\boldsymbol{x}^{0}, \boldsymbol{\xi}\right)\right)$ have the same values.

In PCE-based statistical analysis of EM structures, the stochastic quantity of interest is $R_{j}(\boldsymbol{x})$. The functional form between $R_{j}(\boldsymbol{x})$ and $\boldsymbol{\xi}$ is approximated by the sum of weighted orthogonal basis polynomials in terms of the standard random parameters $\boldsymbol{\xi}$ as follows [36]

$$
R_{j}(\boldsymbol{x})=R_{j}\left(T^{-1}\left(\boldsymbol{x}^{0}, \boldsymbol{\xi}\right)\right)=\sum_{i=0}^{P} a_{i j}\left(\boldsymbol{x}^{0}\right) \Phi_{i}(\boldsymbol{\xi}),
$$

where $\Phi_{i}(\cdot)$ is the generalized polynomial chaos basis function. The optimal bases to construct the multi-variate basis depend on the continuous probability distribution types of the design parameters. For example, Hermite polynomials are optimal for normal distribution while Legendre polynomials correspond to uniform distribution 
[93]. $a_{i j}\left(\boldsymbol{x}^{0}\right)$ is the weighting coefficient for the $i$-th basis function $\Phi_{i}$ of the $j$-th EM response $R_{j}\left(T^{-1}\left(\boldsymbol{x}^{0}, \boldsymbol{\xi}\right)\right) . P+1$ is the number of terms in (5.4), given by [36]

$$
P+1=\frac{(n+D) !}{n ! \cdot D !}
$$

where $n$ is the dimentionality of $\boldsymbol{x}$, and $D$ is the highest polynomial order in the expansion.

In this thesis, we focus on the non-intrusive stochastic collocation scheme (more specifically, the spectral projection approach) to compute the PCE coefficients $a_{i j}\left(\boldsymbol{x}^{0}\right)$. Using the orthogonality condition of the polynomial chaos basis functions $\Phi_{i}(\boldsymbol{\xi})$, the PCE coefficients $a_{i j}\left(\boldsymbol{x}^{0}\right)$ can be found by projection [39]

$$
a_{i j}\left(\boldsymbol{x}^{0}\right)=\frac{\int_{\Omega^{n}} R_{j}\left(T^{-1}\left(\boldsymbol{x}^{0}, \boldsymbol{\xi}\right)\right) \Phi_{i}(\boldsymbol{\xi}) \rho(\boldsymbol{\xi}) d \boldsymbol{\xi}}{\int_{\Omega^{n}} \Phi_{i}^{2}(\boldsymbol{\xi}) \rho(\boldsymbol{\xi}) d \boldsymbol{\xi}}
$$

where $\Omega^{n}$ is the $n$-dimensional space of all possible values of $\boldsymbol{\xi} . \rho(\boldsymbol{\xi})$ is the joint probability density function $(\mathrm{PDF})$ of the standard random parameters $\boldsymbol{\xi}$. The definition of $\rho(\boldsymbol{\xi})$ is given by $\rho(\boldsymbol{\xi})=\prod_{d=1}^{n} f\left(\xi_{d}\right)$, where $f\left(\xi_{d}\right)$ is the PDF of $\xi_{d}$, $d=1,2, \cdots, n$. Equation (5.6) is the same as that in [39] except that the change of the nominal point is considered. As $\boldsymbol{x}^{0}$ changes during yield optimization, the PCE coefficients $a_{i j}$ need to be re-evaluated from iteration to iteration.

The multi-dimensional integration in (5.6) can be evaluated using numerical quadrature, e.g., [39]

$$
\int_{\Omega^{n}} R_{j}\left(T^{-1}\left(\boldsymbol{x}^{0}, \boldsymbol{\xi}\right)\right) \Phi_{i}(\boldsymbol{\xi}) \rho(\boldsymbol{\xi}) d \boldsymbol{\xi} \approx \sum_{l=1}^{M} R_{j}\left(T^{-1}\left(\boldsymbol{x}^{0}, \boldsymbol{\xi}^{(l)}\right)\right) \Phi_{i}\left(\boldsymbol{\xi}^{(l)}\right) w^{(l)}
$$




$$
\int_{\Omega^{n}} \Phi_{i}^{2}(\boldsymbol{\xi}) \rho(\boldsymbol{\xi}) d \boldsymbol{\xi} \approx \sum_{l=1}^{M} \Phi_{i}^{2}\left(\boldsymbol{\xi}^{(l)}\right) w^{(l)},
$$

where $\boldsymbol{\xi}^{(l)}$ and $w^{(l)}$ are the integration quadrature points (also called "nodes") and weights in the " $\xi$-space", respectively. $M$ is the total number of integration quadrature points. $R_{j}\left(T^{-1}\left(\boldsymbol{x}^{0}, \boldsymbol{\xi}^{(l)}\right)\right)$ is the EM response evaluated at the $l$-th sampling point in the original random space. As an example, assuming that the design parameters are independently Gaussian distributed with mean values $x_{1}^{0}, x_{2}^{0}, \cdots, x_{n}^{0}$ and standard deviations $\sigma_{1}, \sigma_{2}, \cdots, \sigma_{n}$, the $l$-th sample in the original space is given by

$$
T^{-1}\left(\boldsymbol{x}^{0}, \boldsymbol{\xi}^{(l)}\right)=\boldsymbol{x}^{0}+\Gamma \boldsymbol{\xi}^{(l)},
$$

where $\Gamma$ is a diagonal matrix containing the standard deviations for all the design parameters, i.e., $\Gamma=\operatorname{diag}\left\{\begin{array}{llll}\sigma_{1} & \sigma_{2} & \cdots & \sigma_{n}\end{array}\right\}$. To reduce the computational costs for multi-dimensional numerical integration in (5.7) and (5.8), sparse grid techniques based on the Smolyak algorithm are typically applied [94]. In many cases this can accurately approximate multi-dimensional integrals with substantially fewer quadrature points.

As the change of $\boldsymbol{x}^{0}$ does not affect the numerical quadrature in (5.8), for notational convenience, we define $b_{i}$ as follows

$$
b_{i}=\sum_{l=1}^{M} \Phi_{i}^{2}\left(\boldsymbol{\xi}^{(l)}\right) w^{(l)} .
$$

$b_{i}$ is problem-independent. It only depends on $M$ and the distribution types of standard random parameters $\boldsymbol{\xi}$. Therefore, $b_{i}$ can be determined before one performs yield optimization. Based on (5.6)-(5.8) and (5.10), the PCE coefficients can be 
computed from

$$
a_{i j}\left(\boldsymbol{x}^{0}\right)=\frac{1}{b_{i}} \sum_{l=1}^{M} R_{j}\left(T^{-1}\left(\boldsymbol{x}^{0}, \boldsymbol{\xi}^{(l)}\right)\right) \Phi_{i}\left(\boldsymbol{\xi}^{(l)}\right) w^{(l)}
$$

where $\Phi_{i}\left(\boldsymbol{\xi}^{(l)}\right)$ and $w^{(l)}$ are constants for yield optimization.

One valuable feature of the PCE approach is that, once the coefficients $a_{i j}\left(\boldsymbol{x}^{0}\right)$ are computed, the statistical properties of the stochastic quantity $R_{j}\left(T^{-1}\left(\boldsymbol{x}^{0}, \boldsymbol{\xi}\right)\right)$, e.g., mean $\mu_{j}\left(\boldsymbol{x}^{0}\right)$ and variance $\sigma_{j}^{2}\left(\boldsymbol{x}^{0}\right)$ can be obtained analytically through these coefficients in a simple closed form [39]

$$
\begin{gathered}
\mu_{j}\left(\boldsymbol{x}^{0}\right)=E\left(R_{j}\right)=a_{0 j}\left(\boldsymbol{x}^{0}\right) \\
\sigma_{j}^{2}\left(\boldsymbol{x}^{0}\right)=E\left[\left(R_{j}-\mu_{j}\right)^{2}\right]=\sum_{i=1}^{P} a_{i j}^{2}\left(\boldsymbol{x}^{0}\right) b_{i} .
\end{gathered}
$$

\subsection{Proposed Non-Surrogate-Based Yield Opti- mization Approach Exploiting PCE}

In this section, our proposed PCE-based yield optimization approach is presented in detail. In the first part, we incorporate the PCE coefficients into the objective function for yield-driven EM optimization. By taking full advantages of the statistical properties provided by the PCE coefficients, the number of EM simulations required to achieve reliable yield representation is reduced. In the second part, we derive the sensitivity formulas of the PCE coefficients w.r.t. the nominal point, followed by the derivation of the sensitivities for the proposed objective function. These sensitivities are then used in gradient-based optimization algorithms to find the optimal yield 
solution iteratively. Finally, the proposed yield optimization process is summarized into a stepwise algorithm.

\subsubsection{Formulation of the Objective Function for Yield-Driven EM Optimization Incorporating PCE Coefficients}

Bandler and Chen presented in [25] a one-sided least $p$-th objective function $U\left(\boldsymbol{x}^{0}\right)$ which was well suited to accelerating yield optimization. The objective function $U\left(\boldsymbol{x}^{0}\right)$ is congregated from the simulated responses related to design specifications for all the circuit outcomes randomly generated around the nominal point. Increase of the yield can be achieved by minimizing $U\left(\boldsymbol{x}^{0}\right)$ since such minimization leads to a better center in the feasible region [26]. In this chapter, we use the formulation in [25] as a starting point and propose a new and different objective function to facilitate yield-driven EM optimization.

In the statistical approach to microwave design, we consider that the random outcomes of the design parameters $\boldsymbol{x}$ are actually spread around the nominal point $\boldsymbol{x}^{0}$ following their statistical distributions and tolerances [27]. The $k$-th random outcome of $\boldsymbol{x}$ can be denoted as

$$
\boldsymbol{x}^{k}=T^{-1}\left(\boldsymbol{x}^{0}, \boldsymbol{\xi}^{k}\right), k=1, \cdots, N
$$

where $N$ is the total number random outcomes of $\boldsymbol{x} . \boldsymbol{\xi}^{k}$ is the vector of standard random parameters that corresponds to $\boldsymbol{x}^{k}$. In this chapter, to distinguish the random outcomes of $\boldsymbol{x}$ in Monte Carlo analysis from the sparse grid samples of $\boldsymbol{x}$ in the PCE approach, we use $k(k=1, \cdots, N)$ to represent the index of random 
outcomes and use $l(l=1, \cdots, M)$ to represent the index of sparse grid samples.

Suppose that $H_{p}(\cdot)$ represents the one-sided least $p$-th function. Following [25], the objective function $U\left(\boldsymbol{x}^{0}\right)$ for the Monte Carlo-based yield optimization is given by $[25]$

$$
U\left(\boldsymbol{x}^{0}\right)=H_{p}\left(\boldsymbol{u}\left(\boldsymbol{x}^{0}\right)\right)
$$

where $\boldsymbol{u}=\left[u_{1}, u_{2}, \cdots, u_{N}\right]^{T}$. The $k$-th component in $\boldsymbol{u}$ is defined as

$$
u_{k}=\alpha_{k} H_{q}\left(\boldsymbol{e}\left(T^{-1}\left(\boldsymbol{x}^{0}, \boldsymbol{\xi}^{k}\right)\right)\right), k=1, \cdots, N
$$

where $q$ is an index indicating the norm used for $\boldsymbol{e}$ while $p$ is an index indicating the norm used for $\boldsymbol{u}$. The specific definition of the least $p$-th norms $(p=1,2, \ldots, \infty)$ can be found in [25]. If we use $p=1$ and $q=1$ (as used in [25]) and take the weighting factor $\alpha_{k}=1$, the objective function $U\left(\boldsymbol{x}^{0}\right)$ will take the following form $[25]$

$$
\begin{aligned}
U\left(\boldsymbol{x}^{0}\right) & =\sum_{k \in K} \sum_{j \in J\left(\boldsymbol{x}^{k}\right)} e_{j}\left(T^{-1}\left(\boldsymbol{x}^{0}, \boldsymbol{\xi}^{k}\right)\right) . \\
J\left(\boldsymbol{x}^{k}\right) & =\left\{j \mid e_{j}\left(T^{-1}\left(\boldsymbol{x}^{0}, \boldsymbol{\xi}^{k}\right)\right)>0\right\} \\
K & =\left\{k \mid J\left(\boldsymbol{x}^{k}\right) \neq \emptyset\right\}
\end{aligned}
$$

The objective function defined in (5.17) typically requires the number of outcomes $N$ to be reasonably sufficient to make the minimization of (5.17) effective. It works efficiently if the computation of responses is by circuit simulations. However, it is not computationally efficient to directly apply (5.17) to yield-driven EM optimization. The reason is that in each iteration of yield optimization, the EM 
simulations need to be done $N$ times, where $N$ is the number of random outcomes of $\boldsymbol{x}$. Further those simulations have to be re-done from iteration to iteration as the nominal point $\boldsymbol{x}^{0}$ is changed, resulting in a large number of EM simulations.

To alleviate this difficulty, we propose a new formulation of the objective function inspired by the fact that the statistical properties of the EM response can be obtained analytically through the PCE coefficients. Provided that the computation of the PCE coefficients is accurate and not too expensive, we can take full advantage of the statistical properties offered by PCE such that the number of EM simulations required to achieve reliable yield representation is reduced. In terms of non-intrusive PCE approaches, it has been shown that the number of integration samples $M$ required to obtain accurate PCE coefficients is much fewer than the number of outcomes $N$ required in Monte Carlo analysis [39]. Thus, if we can use the PCE coefficients properly to formulate a new objective function, the computational costs to achieve reliable yield representation can be reduced and the overall yield optimization process can be expedited. In the subsequent descriptions, we follow this idea to propose our PCE-based yield optimization approach.

As shown in (5.4), for each specification sample $S_{j}$, we have one response $R_{j}\left(T^{-1}\left(\boldsymbol{x}^{0}, \boldsymbol{\xi}\right)\right)$ and one corresponding set of PCE coefficients $a_{i j}, i=0,1, \cdots, P$. In order to incorporate the PCE coefficients into EM-based yield optimization, we need to re-organize the objective function defined in (5.17) into a form where the error element $e_{j}\left(T^{-1}\left(\boldsymbol{x}^{0}, \boldsymbol{\xi}^{k}\right)\right)$ is accumulated first w.r.t. each specification sample $S_{j}$ then w.r.t. each set of design parameters of the outcomes $\boldsymbol{x}^{k}$. By doing this, the statistical properties of the responses computed from the PCE coefficients can 
be exploited. To achieve this, we rewrite the original objective function $U\left(\boldsymbol{x}^{0}\right)$ in (5.17) by swapping the order of the summations, and at the same time re-arrange the elements in $J$ and $K$ as follows

$$
\begin{aligned}
U\left(\boldsymbol{x}^{0}\right) & =\sum_{j \in \bar{J}} \sum_{k \in \bar{K}_{j}} e_{j}\left(T^{-1}\left(\boldsymbol{x}^{0}, \boldsymbol{\xi}^{k}\right)\right), \\
\bar{K}_{j} & =\left\{k \mid e_{j}\left(T^{-1}\left(\boldsymbol{x}^{0}, \boldsymbol{\xi}^{k}\right)\right)>0\right\} \\
\bar{J} & =\left\{j \mid \bar{K}_{j} \neq \emptyset\right\}
\end{aligned}
$$

where $\bar{K}_{j}$ is a set containing all the indices of the outcomes whose responses violate the specification sample $S_{j} . \bar{J}$ is a set containing all the indices of the specification samples which are violated by at least one outcome.

As shown in (5.3), the error element $e_{j}\left(T^{-1}\left(\boldsymbol{x}^{0}, \boldsymbol{\xi}^{k}\right)\right)$ is calculated differently for upper and lower specifications. For convenience of description, we divide the re-organized objective function (5.20) into two parts according to the design specification type of $S_{j}$ as follows

$$
U\left(\boldsymbol{x}^{0}\right)=\sum_{j \in \bar{J}_{u}} \sum_{k \in \bar{K}_{j}} e_{j}\left(T^{-1}\left(\boldsymbol{x}^{0}, \boldsymbol{\xi}^{k}\right)\right)+\sum_{j \in \bar{J}_{l}} \sum_{k \in \bar{K}_{j}} e_{j}\left(T^{-1}\left(\boldsymbol{x}^{0}, \boldsymbol{\xi}^{k}\right)\right),
$$

where $\bar{J}_{u}$ and $\bar{J}_{l}$ contain the indices of the upper and lower specification samples which are violated by at least one outcome, respectively, i.e.,

$$
\begin{gathered}
\bar{J}_{u}=\left\{j \mid j \in \bar{J}, 1 \leq j \leq m_{u}\right\}, \\
\bar{J}_{l}=\left\{j \mid j \in \bar{J}, m_{u}<j \leq m\right\} .
\end{gathered}
$$

It can be noted that $\bar{J}_{u} \cup \bar{J}_{l}=\bar{J}$. 
Let $N_{j}^{\text {fail }}$ be the number of elements in $\bar{K}_{j} . \quad N_{j}^{\text {fail }}$ represents the number of outcomes whose responses fail to satisfy the specification $S_{j}$. If the total number of outcomes $N$ goes to infinity, then the statistical properties of $\sum_{k \in \bar{K}_{j}} e_{j}\left(T^{-1}\left(\boldsymbol{x}^{0}, \boldsymbol{\xi}^{k}\right)\right)$ can be represented more accurately by an integral form as follows

$$
\begin{aligned}
\lim _{N \rightarrow \infty} \frac{1}{N} \sum_{k \in \bar{K}_{j}} e_{j}\left(T^{-1}\left(\boldsymbol{x}^{0}, \boldsymbol{\xi}^{k}\right)\right) \\
=\frac{1}{N} E\left(e_{j}\left(T^{-1}\left(\boldsymbol{x}^{0}, \boldsymbol{\xi}\right)\right) \cdot N_{j}^{\text {fail }}\right. \\
=E\left[\left(R_{j}-S_{j}\right) \mid R_{j}>S_{j}\right] \cdot \frac{N_{j}^{\text {fail }}}{N} \\
=\frac{\int_{S_{j}}^{\infty}\left(R_{j}-S_{j}\right) f\left(R_{j}\right) \mathrm{d} R_{j}}{\int_{S_{j}}^{\infty} f\left(R_{j}\right) \mathrm{d} R_{j}} \cdot \frac{N_{j}^{f a i l}}{N} \\
=\frac{\int_{S_{j}}^{\infty}\left(R_{j}-S_{j}\right) f\left(R_{j}\right) \mathrm{d} R_{j}}{N_{j}^{f a i l} / N} \cdot \frac{N_{j}^{f a i l}}{N} \\
=\int_{S_{j}}^{\infty}\left(R_{j}-S_{j}\right) f\left(R_{j}\right) \mathrm{d} R_{j} \\
=-S_{j} \int_{S_{j}}^{\infty} f\left(R_{j}\right) \mathrm{d} R_{j}+\int_{S_{j}}^{\infty} R_{j} f\left(R_{j}\right) \mathrm{d} R_{j},
\end{aligned}
$$

where $j \in \bar{J}_{u}, R_{j}=R_{j}\left(T^{-1}\left(\boldsymbol{x}^{0}, \boldsymbol{\xi}\right)\right)$, which can be expressed as truncated series expansion as shown in (5.4) if we apply the PCE approach. $E\left[\left(R_{j}-S_{j}\right) \mid R_{j}>S_{j}\right]$ represents the mean value of $\left(R_{j}-S_{j}\right)$ conditional on $R_{j}>S_{j} . f\left(R_{j}\right)$ denotes the $\mathrm{PDF}$ of $R_{j}$.

Similarly, the statistical properties of $\sum_{k \in \bar{K}_{j}} e_{j}\left(T^{-1}\left(\boldsymbol{x}^{0}, \boldsymbol{\xi}^{k}\right)\right), j \in \bar{J}_{l}$, can be repre- 
sented more accurately by an integral form as follows

$$
\lim _{N \rightarrow \infty} \frac{1}{N} \sum_{k \in \bar{K}_{j}} e_{j}\left(T^{-1}\left(\boldsymbol{x}^{0}, \boldsymbol{\xi}^{k}\right)\right)=S_{j} \int_{-\infty}^{S_{j}} f\left(R_{j}\right) \mathrm{d} R_{j}-\int_{-\infty}^{S_{j}} R_{j} f\left(R_{j}\right) \mathrm{d} R_{j} .
$$

It is noted that (5.26) and (5.27) have different intervals for the integration, i.e., (5.26) has interval $\left[S_{j}, \infty\right)$ while $(5.27)$ has interval $\left(-\infty, S_{j}\right]$.

For notational convenience, we define $\bar{u}_{j}\left(\boldsymbol{x}^{0}\right)$ as a yield indicator w.r.t. the specification sample $S_{j}$ as follows

$$
\bar{u}_{j}\left(\boldsymbol{x}^{0}\right)=\left\{\begin{array}{l}
-S_{j} \int_{S_{j}}^{\infty} f\left(R_{j}\right) \mathrm{d} R_{j}+\int_{S_{j}}^{\infty} R_{j} f\left(R_{j}\right) \mathrm{d} R_{j}, j \in \bar{J}_{u}, \\
S_{j} \int_{-\infty}^{S_{j}} f\left(R_{j}\right) \mathrm{d} R_{j}-\int_{-\infty}^{S_{j}} R_{j} f\left(R_{j}\right) \mathrm{d} R_{j}, j \in \bar{J}_{l} .
\end{array}\right.
$$

$\bar{u}_{j}\left(\boldsymbol{x}^{0}\right)$ can be regarded as an indicator of the yield because a small value of $\bar{u}_{j}\left(\boldsymbol{x}^{0}\right)$ basically represents that there are few outcomes whose responses violate the specification sample $S_{j}$, indicating a high yield. Therefore, reducing $\bar{u}_{j}\left(\boldsymbol{x}^{0}\right)$ is expected to lead to an increase of the yield.

Given $\mu_{j}, \sigma_{j}^{2}$, and higher moments of $R_{j}[93], f\left(R_{j}\right)$ in (5.28) can be approximated using existing PDF estimation techniques, e.g., the moment matching technique [113] and the maximum entropy technique [114]. Then the integrals in $\bar{u}_{j}\left(\boldsymbol{x}^{0}\right)$ can be evaluated by applying the Gauss quadratures developed in [115]. In this thesis, to analytically relate $\bar{u}_{j}\left(\boldsymbol{x}^{0}\right)$ to the nominal point through the PCE coefficients, we consider a special case where the EM response $R_{j}$ follows normal distribution with $\mu_{j}$ and $\sigma_{j}$ as its mean and standard deviation, respectively. In the following de- 
scriptions, we show how $\bar{u}_{j}\left(\boldsymbol{x}^{0}\right)$ is analytically related to the nominal point through PCE coefficients under such consideration.

Based on (5.12) and (5.13), the first term of $\bar{u}_{j}\left(\boldsymbol{x}^{0}\right), j \in \bar{J}_{u}$, can be related to the nominal point $\boldsymbol{x}^{0}$ through PCE coefficients as follows

$$
-S_{j} \int_{S_{j}}^{\infty} f\left(R_{j}\right) \mathrm{d} R_{j}=S_{j}\left(\phi\left(\frac{S_{j}-a_{0 j}\left(\boldsymbol{x}^{0}\right)}{\sqrt{\sum_{i=1}^{P} a_{i j}^{2}\left(\boldsymbol{x}^{0}\right) b_{i}}}\right)-1\right),
$$

where $\phi(\cdot)$ is the cumulative distribution function (CDF) of the standard normal distribution. $a_{0 j}$ and $a_{i j}$ are PCE coefficients as functions of yield optimization variables $\boldsymbol{x}^{0}$.

For notational convenience, let $\gamma_{j}\left(\boldsymbol{x}^{0}\right)$ be defined as

$$
\gamma_{j}\left(\boldsymbol{x}^{0}\right)=\frac{S_{j}-a_{0 j}\left(\boldsymbol{x}^{0}\right)}{\sqrt{\sum_{i=1}^{P} a_{i j}^{2}\left(\boldsymbol{x}^{0}\right) b_{i}}}, \quad j \in \bar{J}_{u} \cup \bar{J}_{l} .
$$

$\phi\left(\gamma_{j}\left(\boldsymbol{x}^{0}\right)\right)$ represents the probability that $R_{j}$ satisfies the specification $S_{j}$ in case $j \in \bar{J}_{u}$ or violates $S_{j}$ in case $j \in \bar{J}_{l}$.

To analytically relate the second term of $\bar{u}_{j}\left(\boldsymbol{x}^{0}\right), j \in \bar{J}_{u}$, to the nominal point through PCE coefficients, we are interested in finding the mean value of the EM response $R_{j}$ under the condition that $R_{j}$ violates the upper specification $S_{j}$. Let $E\left(R_{j} \mid\right.$ $R_{j}>S_{j}$ ) be the mean value of $R_{j}$ conditional on $R_{j}>S_{j}$. Then $\int_{S_{j}}^{\infty} R_{j} f\left(R_{j}\right) \mathrm{d} R_{j}$ 
can be analytically related to the nominal point $\boldsymbol{x}^{0}$ as follows [116]

$$
\begin{aligned}
\int_{S_{j}}^{\infty} & R_{j} f\left(R_{j}\right) \mathrm{d} R_{j} \\
& =E\left(R_{j} \mid R_{j}>S_{j}\right) \cdot \int_{S_{j}}^{\infty} f\left(R_{j}\right) \mathrm{d} R_{j} \\
& =\mu_{j}\left(1-\phi\left(\gamma_{j}\left(\boldsymbol{x}^{0}\right)\right)\right)+\frac{1}{\sqrt{2 \pi}} \sigma_{j} \cdot e^{-\frac{1}{2} \gamma_{j}^{2}\left(\boldsymbol{x}^{0}\right)},
\end{aligned}
$$

where $\mu_{j}$ and $\sigma_{j}$ can be computed from PCE coefficients as given by (5.12) and (5.13).

The PCE coefficients are incorporated into $\bar{u}_{j}\left(\boldsymbol{x}^{0}\right)$ for the upper specifications by substituting (5.12)-(5.13) and (5.29)-(5.31) into $\bar{u}_{j}\left(\boldsymbol{x}^{0}\right), j \in \bar{J}_{u}$, as follows

$$
\bar{u}_{j}\left(\boldsymbol{x}^{0}\right)=\sqrt{\sum_{i=1}^{P} a_{i j}^{2}\left(\boldsymbol{x}^{0}\right) b_{i}} \cdot\left[\left(\phi\left(\gamma_{j}\left(\boldsymbol{x}^{0}\right)\right)-1\right) \gamma_{j}\left(\boldsymbol{x}^{0}\right)+\frac{1}{\sqrt{2 \pi}} e^{-\frac{1}{2} \gamma_{j}^{2}\left(\boldsymbol{x}^{0}\right)}\right] .
$$

Following similar derivations, we can incorporate the PCE coefficients into $\bar{u}_{j}\left(\boldsymbol{x}^{0}\right)$ for the lower specifications, $j \in \bar{J}_{l}$, as follows

$$
\bar{u}_{j}\left(\boldsymbol{x}^{0}\right)=\sqrt{\sum_{i=1}^{P} a_{i j}^{2}\left(\boldsymbol{x}^{0}\right) b_{i}} \cdot\left[\phi\left(\gamma_{j}\left(\boldsymbol{x}^{0}\right)\right) \gamma_{j}\left(\boldsymbol{x}^{0}\right)+\frac{1}{\sqrt{2 \pi}} e^{-\frac{1}{2} \gamma_{j}^{2}\left(\boldsymbol{x}^{0}\right)}\right] .
$$

The proposed PCE-based objective function $\bar{U}\left(\boldsymbol{x}^{0}\right)$ for yield-driven EM optimization including both upper and lower specifications is given by

$$
\bar{U}\left(\boldsymbol{x}^{0}\right)=\sum_{j \in \bar{J}_{u}} \bar{u}_{j}\left(\boldsymbol{x}^{0}\right)+\sum_{j \in \bar{J}_{l}} \bar{u}_{j}\left(\boldsymbol{x}^{0}\right),
$$

where $\bar{u}_{j}\left(\boldsymbol{x}^{0}\right)$ is given by (5.32) and (5.33) for upper $\left(j \in \bar{J}_{u}\right)$ and lower specifications $\left(j \in \bar{J}_{l}\right)$, respectively. By substituting (5.32) and (5.33) into (5.34), the proposed 
optimization objective function can be written in a more convenient form

$$
\bar{U}\left(\boldsymbol{x}^{0}\right)=\sum_{j \in \bar{J}} V_{j}\left(\boldsymbol{x}^{0}\right)+\sum_{j \in \bar{J}_{u}}\left(a_{0 j}\left(\boldsymbol{x}^{0}\right)-S_{j}\right),
$$

where $V_{j}\left(\boldsymbol{x}^{0}\right)$ is computed from the PCE coefficients as follows

$$
V_{j}\left(\boldsymbol{x}^{0}\right)=\sqrt{\sum_{i=1}^{P} a_{i j}^{2}\left(\boldsymbol{x}^{0}\right) b_{i}} \cdot\left[\phi\left(\gamma_{j}\left(\boldsymbol{x}^{0}\right)\right) \gamma_{j}\left(\boldsymbol{x}^{0}\right)+\frac{1}{\sqrt{2 \pi}} e^{-\frac{1}{2} \gamma_{j}^{2}\left(\boldsymbol{x}^{0}\right)}\right] .
$$

Notice that $a_{i j}\left(\boldsymbol{x}^{0}\right)$ are PCE coefficients, and $\gamma_{j}\left(\boldsymbol{x}^{0}\right)$ is computed from the PCE coefficients $a_{i j}\left(\boldsymbol{x}^{0}\right)$ as shown in (5.30).

It is pointed out that through (5.20)-(5.36), we have changed the original objective function defined in (5.17) into a new form where the PCE coefficients are incorporated. The PCE coefficients are able to provide the statistical properties and approximate the PDF of the EM response with fewer EM samples than that required by Monte Carlo analysis. Therefore, the new PCE-based formulation requires fewer EM simulations to obtain accurate yield representation than the original formulation (which is based on assembling the responses evaluated at Monte Carlo samples). This advantage allows our proposed objective function to be able to facilitate the overall EM-based yield optimization process more efficiently.

\subsubsection{Derivation of Sensitivity Formulas for the Proposed Objective Function}

To use the proposed objective function formulated above in yield-driven EM optimization, the derivatives of the proposed objective function $\bar{U}\left(\boldsymbol{x}^{0}\right)$ w.r.t. the varying nominal point $\boldsymbol{x}^{0}$ need to be derived so that a gradient-based optimization algorithm 
(e.g., quasi-Newton method) can be employed. In this subsection, we first derive the sensitivity formulas of PCE coefficients w.r.t. $\boldsymbol{x}^{0}$, then derive the sensitivities for the objective function based on the sensitivity formulas of the PCE coefficients.

At each iteration of the proposed yield optimization algorithm, a set of EM design parameters $\mathcal{X}$ are generated around the current nominal point $\boldsymbol{x}^{0}$ following the sparse grid technique, i.e.,

$$
\mathcal{X}=\left\{T^{-1}\left(\boldsymbol{x}^{0}, \boldsymbol{\xi}^{(1)}\right), \cdots, T^{-1}\left(\boldsymbol{x}^{0}, \boldsymbol{\xi}^{(M)}\right)\right\}
$$

Then the PCE coefficients are numerically evaluated using the EM responses simulated at these design parameters. The sparse grid samples are formed based on the Smolyak algorithm [117], which selectively combines the tensor products of lower order quadrature rules to cover the parameter space more efficiently [39]. As illustrated in Fig. 5.1, as the nominal point moves from $\boldsymbol{x}^{0}$ to $\boldsymbol{x}_{\text {new }}^{0}$, the sparse grid samples in the original parameter space move accordingly, forming a new set of sparse grid samples $\mathcal{X}_{\text {new }}$. In other words, the movement of the nominal point affects the location of every sparse grid point in the original parameter space, which in turn affects each PCE coefficient $a_{i j}$.

In this work, we consider the design parameters to be Gaussian (normal) distributed and that the standard deviation is $\sigma_{d}$ for the $d$-th design parameter, 


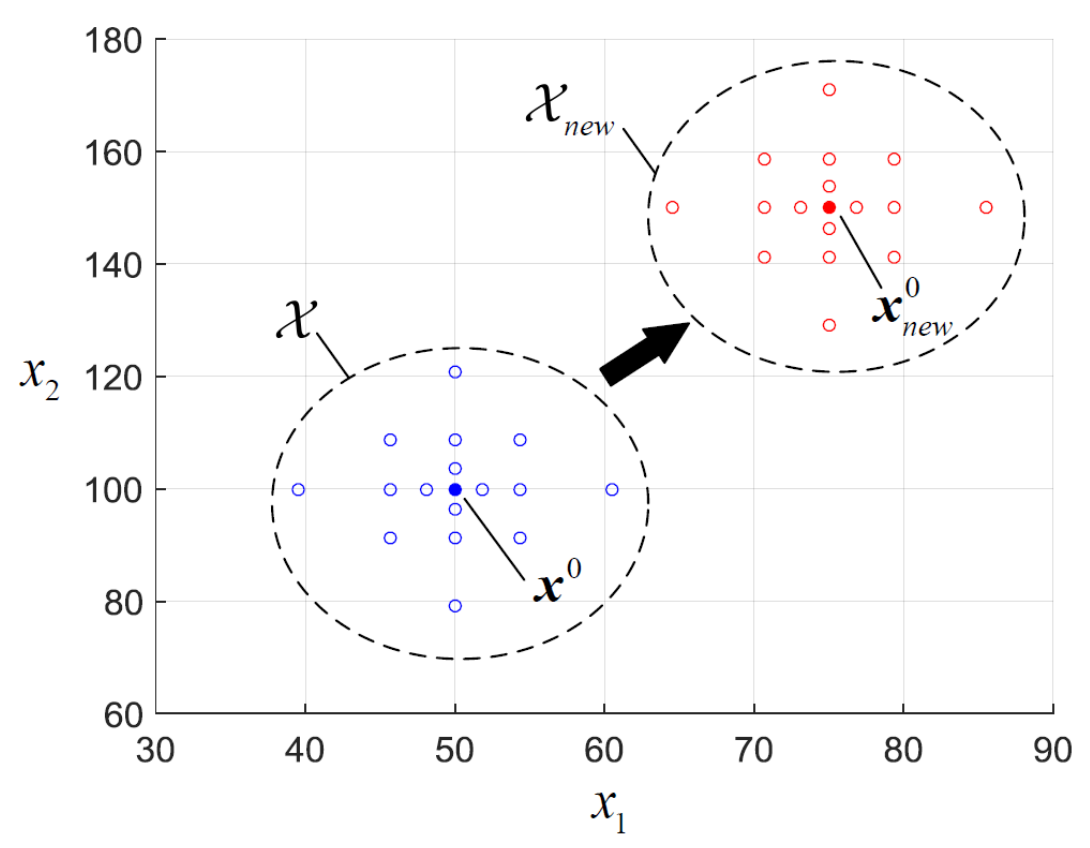

Figure 5.1: Illustration of the movement of sparse grid samples in PCE between two consecutive iterations during yield optimization. The two black dots represent the nominal points between two successive iterations. The circles represent the integration samples around the nominal points following the sparse grid technique. In yield optimization, the nominal point $\boldsymbol{x}^{0}$ is a variable which is updated iteratively. As the nominal point moves from $\boldsymbol{x}^{0}$ to $\boldsymbol{x}_{\text {new }}^{0}$, all the sparse grid samples move accordingly.

$d=1,2, \cdots, n$. Then the reverse transformation in (5.9) takes the following form

$$
T^{-1}\left(\boldsymbol{x}^{0}, \boldsymbol{\xi}^{(l)}\right)=\left[\begin{array}{c}
x_{1}^{0} \\
x_{2}^{0} \\
\vdots \\
x_{n}^{0}
\end{array}\right]+\left[\begin{array}{c}
\sigma_{1} \xi_{1}^{(l)} \\
\sigma_{2} \xi_{2}^{(l)} \\
\vdots \\
\sigma_{n} \xi_{n}^{(l)}
\end{array}\right]
$$

where $l=1, \cdots, M$. Note that $\sigma_{d}$ and $\xi_{d}^{(l)}$ are predetermined constants for yield 
optimization.

Based on (5.11) and (5.38), we can obtain the derivatives of each PCE coefficient $a_{i j}\left(\boldsymbol{x}^{0}\right)$ w.r.t. the nominal point $\boldsymbol{x}^{0}$ as follows

$$
\frac{\partial a_{i j}\left(\boldsymbol{x}^{0}\right)}{\partial \boldsymbol{x}^{0}}=\left.\frac{1}{b_{i}} \sum_{l=1}^{M} \Phi_{i}\left(\boldsymbol{\xi}^{(l)}\right) w^{(l)} \frac{\partial R_{j}(\boldsymbol{x})}{\partial \boldsymbol{x}}\right|_{\boldsymbol{x}=\boldsymbol{x}^{(l)}},
$$

where $\boldsymbol{x}^{(l)}=T^{-1}\left(\boldsymbol{x}^{0}, \boldsymbol{\xi}^{(l)}\right) .\left.\frac{\partial R_{j}(\boldsymbol{x})}{\partial \boldsymbol{x}}\right|_{\boldsymbol{x}=\boldsymbol{x}^{(l)}}$ represents the EM sensitivities of $R_{j}(\boldsymbol{x})$ evaluated at the $l$-th sparse grid point $\boldsymbol{x}^{(l)}$ in the original parameter space. These EM sensitivities can be obtained from existing EM solvers with sensitivity analysis feature. $b_{i}, \Phi_{i}\left(\boldsymbol{\xi}^{(l)}\right)$ and $w^{(l)}$ are predetermined constants for yield optimization.

From (5.35) and (5.36) we can deduce the derivatives of the proposed objective function $\bar{U}\left(\boldsymbol{x}^{0}\right)$ w.r.t. each PCE coefficient $a_{i j}$ as follows

$$
\frac{\partial \bar{U}\left(\boldsymbol{x}^{0}\right)}{\partial a_{i j}}= \begin{cases}1-\phi\left(\gamma_{j}\left(\boldsymbol{x}^{0}\right)\right), & \text { for } i=0, j \in \bar{J}_{u}, \\ -\phi\left(\gamma_{j}\left(\boldsymbol{x}^{0}\right)\right), & \text { for } i=0, j \in \bar{J}_{l}, \\ 2 a_{i j} b_{i} \frac{1}{\sqrt{2 \pi}} \frac{1}{\sqrt{\sum_{p=1}^{P} a_{p j}^{2}\left(\boldsymbol{x}^{0}\right) b_{p}}} \cdot e^{-\frac{1}{2} \gamma_{j}^{2}\left(\boldsymbol{x}^{0}\right)}, & \text { for } i=1, \cdots, P, j \in \bar{J}_{u} \cup \bar{J}_{l} .\end{cases}
$$

The derivatives of the proposed objective function $\bar{U}\left(\boldsymbol{x}^{0}\right)$ w.r.t. the varying nominal point $\boldsymbol{x}^{0}$ can be found by

$$
\frac{\partial \bar{U}\left(\boldsymbol{x}^{0}\right)}{\partial \boldsymbol{x}^{0}}=\sum_{i=0}^{P} \sum_{j \in \bar{J}} \frac{\partial \bar{U}\left(\boldsymbol{x}^{0}\right)}{\partial a_{i j}} \frac{\partial a_{i j}\left(\boldsymbol{x}^{0}\right)}{\partial \boldsymbol{x}^{0}},
$$


where $\frac{\partial \bar{U}\left(\boldsymbol{x}^{0}\right)}{\partial a_{i j}}$ and $\frac{\partial a_{i j}\left(\boldsymbol{x}^{0}\right)}{\partial \boldsymbol{x}^{0}}$ are given by (5.39) and (5.40), respectively.

\subsubsection{PCE-based Yield Optimization Algorithm}

In this subsection, we provide the detailed algorithm for the proposed PCE-based yield-driven EM optimization.

Firstly, a set of $M$ samples $\left\{\boldsymbol{\xi}^{(1)}, \boldsymbol{\xi}^{(2)}, \cdots, \boldsymbol{\xi}^{(M)}\right\}$ are generated in the " $\boldsymbol{\xi}$-space" following the rules of the sparse grid technique. These samples are generated only once and would be reused in the subsequent iterations. The distribution pattern of the samples follows the rules of sparse grid techniques [94]. $M$ is related to the accuracy level in sparse grid techniques. Increasing the accuracy level improves the multi-dimensional integration accuracy, but results in a larger $M$ and thus requires more EM simulations. It is recommended that one fulfills the PCE model construction at the initial nominal point before performing yield optimization for a specific EM structure to obtain the required minimal accuracy level. The minimal accuracy level can be obtained by starting from a small value and increasing it gradually until no significant integration difference can be observed between two successive accuracy levels.

Next, the $M$ samples are transformed into EM geometrical parameter samples $\left\{T^{-1}\left(\boldsymbol{x}^{0}, \boldsymbol{\xi}^{(1)}\right), T^{-1}\left(\boldsymbol{x}^{0}, \boldsymbol{\xi}^{(2)}\right), \cdots, T^{-1}\left(\boldsymbol{x}^{0}, \boldsymbol{\xi}^{(M)}\right)\right\}$ using (5.38). Then an existing EM simulator is driven to evaluate the EM responses $R_{j}\left(T^{-1}\left(\boldsymbol{x}^{0}, \boldsymbol{\xi}^{(l)}\right)\right)$ and the EM sen-

sitivities $\left.\frac{\partial R_{j}(\boldsymbol{x})}{\partial \boldsymbol{x}}\right|_{\boldsymbol{x}=\boldsymbol{x}^{(l)}}$, for $l=1,2, \cdots, M$. Since the responses and the sensitivities for different samples can be obtained independently, we evaluate the EM responses and the EM sensitivities at those $M$ samples in parallel [118]. By using this parallel 
computation scheme, we achieve additional speed up for EM-based yield optimization. Then the PCE coefficients $a_{i j}\left(\boldsymbol{x}^{0}\right)$ are numerically evaluated using (5.6)-(5.8) and the derivatives of each PCE coefficient w.r.t. the nominal point are evaluated according to (5.39). After that, the proposed PCE-based objective function $\bar{U}\left(\boldsymbol{x}^{0}\right)$ is evaluated using (5.35) and (5.36), while the derivatives $\frac{\partial \bar{U}\left(\boldsymbol{x}^{0}\right)}{\partial \boldsymbol{x}^{0}}$ are evaluated following (5.39)-(5.41).

Finally, $\bar{U}\left(\boldsymbol{x}^{0}\right)$ and $\frac{\partial \bar{U}\left(\boldsymbol{x}^{0}\right)}{\partial \boldsymbol{x}^{0}}$ are used in a gradient-based optimization algorithm (such as quasi-Newton method) to find the update direction and suitable step size for the change of the nominal point from the current point $\boldsymbol{x}^{0}$ to a new point. Let the new nominal point be denoted as $\boldsymbol{x}_{\text {new }}^{0}$. The optimization terminates if the iteration counter $N_{\text {iter }}$ exceeds the maximum iteration count $N_{i t e r}^{\max }$ or the difference of $\boldsymbol{x}^{0}$ between subsequent iterations is sufficiently small, i.e.,

$$
\begin{gathered}
N_{\text {iter }}>N_{\text {iter }}^{\max }, \\
\text { or }\left\|\boldsymbol{x}_{\text {new }}^{0}-\boldsymbol{x}^{0}\right\|<\varepsilon,
\end{gathered}
$$

where $N_{\text {iter }}=0,1, \cdots \varepsilon \varepsilon$ is a user-defined threshold. Fig. 5.2 shows the flow chart of the proposed PCE-based yield optimization algorithm. The proposed algorithm can be summarized into the following steps.

Step 1) Set the initial nominal point $\boldsymbol{x}_{i n i}^{0}$ as the starting point for yield optimization, i.e., $\boldsymbol{x}^{0}=\boldsymbol{x}_{i n i}^{0}$. Typically, $\boldsymbol{x}_{i n i}^{0}$ should be the optimal solution of nominal EM optimization. Initialize the iteration counter $N_{i t e r}$ as 0 . Set the maximum iteration number $N_{i t e r}^{\max }$ and the stopping criteria $\varepsilon$. 
Step 2) Generate $M$ samples $\left\{\boldsymbol{\xi}^{(1)}, \boldsymbol{\xi}^{(2)}, \cdots, \boldsymbol{\xi}^{(M)}\right\}$ in the " $\boldsymbol{\xi}$-space" following the rules of the sparse grid technique.

Step 3) Transform $\left\{\boldsymbol{\xi}^{(1)}, \boldsymbol{\xi}^{(2)}, \cdots, \boldsymbol{\xi}^{(M)}\right\}$ into a set of EM geometrical parameter samples around the nominal point using (5.38).

Step 4) Evaluate the EM responses $R_{j}\left(T^{-1}\left(\boldsymbol{x}^{0}, \boldsymbol{\xi}^{(l)}\right)\right)$ and the EM sensitivities $\frac{\partial R_{j}(\boldsymbol{x})}{\partial \boldsymbol{x}^{(l)}}$, for $l=1,2, \cdots, M$, in parallel.

Step 5) Numerically evaluate the PCE coefficients $a_{i j}\left(\boldsymbol{x}^{0}\right)$ using (5.6)-(5.8) and the derivatives of each PCE coefficient w.r.t. the nominal point using (5.39).

Step 6) Evaluate the proposed PCE-based objective function $\bar{U}\left(\boldsymbol{x}^{0}\right)$ defined in $(5.35)-(5.36)$.

Step 7) Evaluate the derivatives of the proposed objective function $\bar{U}\left(\boldsymbol{x}^{0}\right)$ w.r.t. the nominal point $\boldsymbol{x}^{0}$ following (5.39)-(5.41).

Step 8) Find the update direction and suitable step size for the current nominal point using gradient-based optimization algorithm (e.g, quasi-Newton method), find the new nominal point $\boldsymbol{x}_{\text {new }}^{0}$.

Step 9) If (5.42) or (5.43) is satisfied, go to step 10). Otherwise, update the nominal point $\boldsymbol{x}^{0}=\boldsymbol{x}_{\text {new }}^{0}$, update the iteration count $N_{\text {iter }}=N_{\text {iter }}+1$ and go to step 3).

Step 10) Obtain the final optimal yield solution $\boldsymbol{x}^{*}=\boldsymbol{x}_{n e w}^{0}$. Stop the optimization process. 


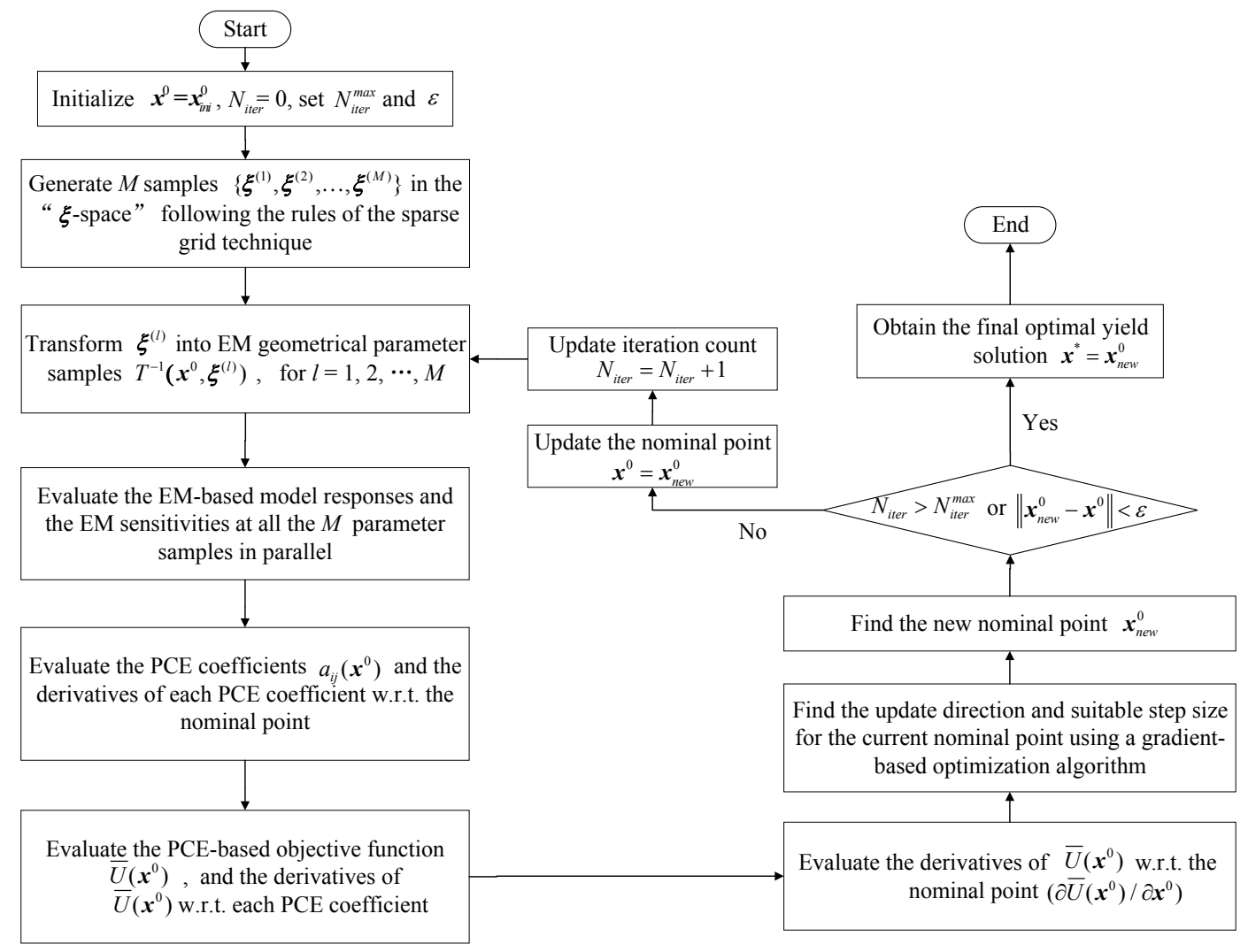

Figure 5.2: Flow chart of the proposed PCE-based yield optimization algorithm.

\subsection{Examples}

\subsubsection{Yield Optimization of a Waveguide K-Band Bandpass Filter}

The first example under consideration is a waveguide K-band bandpass filter described in $A D S$ EMPro tutorial document. The structure of the filter is shown in Fig. 5.3. The section of the waveguide where the filter is constructed is 10.668 $\mathrm{mm} \times 4.318 \mathrm{~mm}(\mathrm{WR}-42)$. The heights of three cylindrical posts are all $4.318 \mathrm{~mm}$. $r_{1}$ is the radius of the two posts on the side while $r_{2}$ is the radius of the post in the middle. The two resonators placed between the cylindrical posts are of equal 


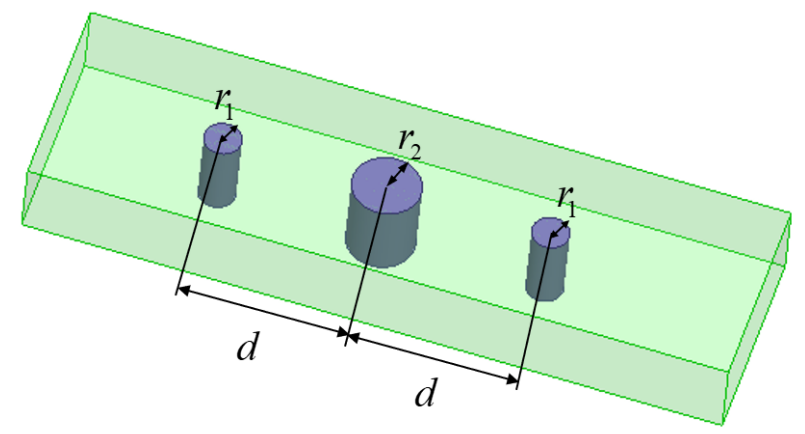

Figure 5.3: The structure of the K-band bandpass filter example for EM simulation and yield optimization. The three design parameters of the filter are $\boldsymbol{x}=\left[\begin{array}{lll}r_{1} & r_{2} & d\end{array}\right]^{T}$.

length $d$. The design parameters are $\boldsymbol{x}=\left[\begin{array}{lll}r_{1} & r_{2} & d\end{array}\right]^{T}(\mathrm{~mm})$. The design specifications are given by $\left|S_{11}\right| \leq-15 \mathrm{~dB}$, in the frequency range from $24.935 \mathrm{GHz}$ to 25.065 $\mathrm{GHz}$, and $\left|S_{11}\right| \geq-1 \mathrm{~dB}$, in the frequency ranges from $24 \mathrm{GHz}$ to $24.75 \mathrm{GHz}$ and from $25.25 \mathrm{GHz}$ to $26 \mathrm{GHz}$. The optimal nominal solution obtained by performing

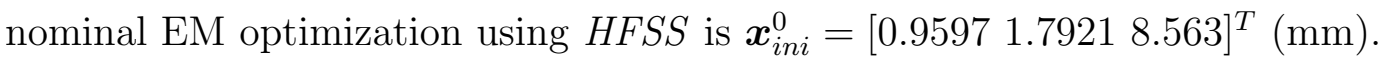

For yield analysis and optimization, we assume independent normal distribution for the design parameters, with the standard deviation being 5 microns. Both the Monte Carlo-based yield optimization approach presented in [25] and the proposed PCE-based yield optimization approach are used to optimize the yield. For the proposed approach, Hermite polynomials are used and the polynomial chaos expansion is truncated at total order $D=2$. The one-dimensional Kronrod-Patterson [94] quadrature rule is used to form a three dimensional total-order grid. An integration accuracy level 3 is required for this example, which results in the total number of quadrature points $M$ being 19 . To find the reasonable value of $N$ for the Monte Carlo-based yield optimization approach, we run a Monte Carlo analysis to estimate 
the yield at the initial nominal point. By varying the number of random samples, a convergence on the yield value is observed when $N=50$. For comparison purpose, we use $N=19, N=30$ and $N=50$ for the Monte Carlo-based yield optimization approach. Quasi-Newton method is used as the gradient-based optimization algorithm to find the optimal yield solution iteratively for both approaches. At each iteration of optimization, HFSS is driven to evaluate the EM responses and the EM sensitivities at all the parameter samples in parallel. To find the optimal yield solutions, we set the maximum number of iterations to be a large number to allow both optimization approaches to converge. When the optimization process terminates, we verify the yield at the optimal yield solution by running a Monte Carlo analysis with 100 random samples for the two approaches.

For this example, we also perform yield optimization using a third approach, i.e., the $A D S$ internal yield optimization tool with EMPro. A parametrized 3D structure of the K-band bandbass filter is created using EMPro. Then nominal EM optimization is performed in $A D S$ to find an optimal nominal solution. Two nominal solutions that can meet the design specifications are found, i.e., $\boldsymbol{x}_{i n i}^{0}=$

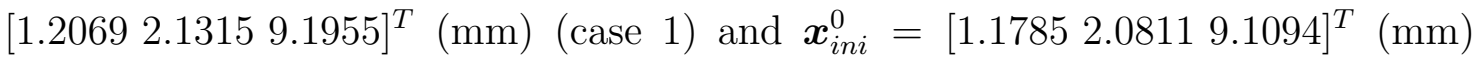
(case 2). Following that, yield estimation is conducted using the yield estimation tool in $A D S$ for both cases. Finally, we take these two nominal solutions as starting points and use the build-in yield optimization tool in $A D S$ to optimize the yield. We stop the yield optimization process until no significant yield increase can be observed.

Table 5.1 summarizes the yield optimization results of three yield optimization 
Table 5.1: Comparison of Yield Optimization Results for the K-Band Bandpass Filter

\begin{tabular}{c|c|c|c|c|c}
\hline \hline $\begin{array}{c}\text { Yield } \\
\text { optimization } \\
\text { approach }\end{array}$ & $\begin{array}{c}\text { Number of EM } \\
\text { simulations } \\
\text { per iteration }\end{array}$ & $\begin{array}{c}\text { Initial } \\
\text { yield }\end{array}$ & $\begin{array}{c}\text { Final } \\
\text { yield }\end{array}$ & $\begin{array}{c}\text { Total Number } \\
\text { of EM } \\
\text { simulations }\end{array}$ & $\begin{array}{c}\text { Total } \\
\text { CPU } \\
\text { time }\end{array}$ \\
\hline $\begin{array}{c}\text { Monte } \\
\text { Carlo-based }\end{array}$ & 19 & $41 \%$ & $52 \%$ & 247 & $2 \mathrm{~h} 25 \mathrm{~min}$ \\
\cline { 2 - 6 } & 30 & $41 \%$ & $56 \%$ & 1050 & $10 \mathrm{~h} 17 \mathrm{~min}$ \\
\hline Proposed & 19 & $41 \%$ & $59 \%$ & 1700 & $16 \mathrm{~h} 39 \mathrm{~min}$ \\
\hline $\begin{array}{c}\text { ADS yield } \\
\text { optimization } \\
\text { (case 1) }\end{array}$ & N/A & $33 \%$ & $49 \%$ & 3350 & $32 \mathrm{~h} 48 \mathrm{~min}$ \\
\hline $\begin{array}{c}\text { ADS yield } \\
\text { optimization } \\
\text { (case 2) }\end{array}$ & N/A & $35 \%$ & $47 \%$ & 3180 & $31 \mathrm{~h} 9 \mathrm{~min}$ \\
\hline \hline
\end{tabular}

approaches for this example. As can be seen from the table, all the three approaches achieve improvements of the yield. However, the total number of EM simulations and the total CPU time required by the Monte Carlo-based yield optimization approach and the proposed approach are much fewer than that required by the build-in yield optimizer in $A D S$. The reason for this is that the Monte Carlo-based yield optimization approach and the proposed approach use sensitivity information and gradient-based optimization algorithms in the yield optimization process. From Table 5.1 it can also be observed that to achieve a similar yield increase the proposed PCE-based approach requires much fewer EM simulations (and thus much shorter CPU time) than the Monte Carlo-based yield optimization approach. This 
Table 5.2: Comparison of Yield Improvements With Similar Number of EM Simulations for the K-Band Bandpass Filter

\begin{tabular}{c|c|c|c|c}
\hline \hline $\begin{array}{c}\text { Yield } \\
\text { optimization } \\
\text { approach }\end{array}$ & $\begin{array}{c}\text { Number of EM } \\
\text { simulations } \\
\text { per iteration }\end{array}$ & Initial yield & Final yield & $\begin{array}{c}\text { Total number } \\
\text { of EM } \\
\text { simulations }\end{array}$ \\
\hline $\begin{array}{c}\text { Monte } \\
\text { Carlo-based }\end{array}$ & 30 & $41 \%$ & $53 \%$ & 540 \\
\cline { 2 - 5 } Proposed & 50 & $41 \%$ & $55 \%$ & 550 \\
\hline \hline
\end{tabular}

is because by incorporating the PCE coefficients into the formulation of the yield optimization objective function, the proposed approach requires fewer EM simulations to find the effective direction and suitable step size for the update of the nominal point at each iteration of optimization than the Monte Carlo-based yield optimization approach. Fig. 5.4 shows the yield before and after optimization using the proposed approach for the K-band bandpass filter. The optimal yield solution found by the proposed approach is $[0.96431 .77798 .554]^{T}(\mathrm{~mm})$.

To further demonstrate the advantages of the proposed approach, we perform another numerical experiment by deliberately stopping the Monte Carlo-based yield optimizations at a certain iteration such that the total number of EM simulations during optimization is similar to that in the proposed approach. Table 5.2 shows the comparison of yield improvements under this consideration. It can be seen from the table that using similar number of EM simulations, the proposed approach achieves a greater yield improvement than the Monte Carlo-based yield optimization approach. The reason for this is that the proposed PCE-based approach provides 
more effective direction and step size for the update of the nominal point than that the Monte Carlo-based yield optimization approach provides. As a result, with similar number of EM simulations, the proposed approach is able to provide more promising yield increase than the Monte Carlo-based yield optimization approach.

\subsubsection{Yield Optimization of a Waveguide Bandpass Filter with Fractal-Shaped Irises}

In the second example, we consider a waveguide bandpass filter with fractal-shaped

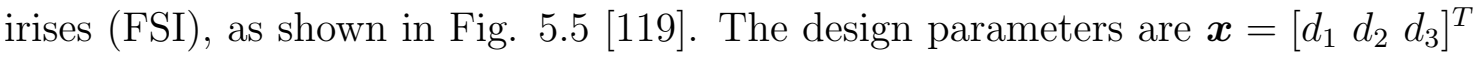
$(\mathrm{mm})$, where $d_{1}, d_{2}$ and $d_{3}$ represent the distances between the first, second, and third pair of symmetrical irises, respectively. An example of the geometrical dimensions of the fractal-shaped irises are given by Fig. 5.6. The section of the waveguide is $a=22.86 \mathrm{~mm}, b=10.16 \mathrm{~mm}$ (WR-90). The design specification for this filter is given by $\left|S_{11}\right| \leq-22 \mathrm{~dB}$, in the frequency range from $9.2 \mathrm{GHz}$ to $9.8 \mathrm{GHz}$. The optimal nominal solution $\boldsymbol{x}_{i n i}^{0}=[9.34444 .92033 .7423]^{T}(\mathrm{~mm})$ is obtained by performing nominal EM optimization [120].

We assume independent normal distributions for all design parameters to allow yield estimation and optimization. The standard deviation for each design parameter is assumed to be 20 microns. The proposed approach and the Monte Carlo-based yield optimization approach are both used to optimize the yield. For the proposed approach, Hermite polynomials are used and the integration accuracy level required is 3 for the sparse grid technique. This results in the number of sparse grid samples $M=19$. For the Monte Carlo-based yield optimization approach, it is found that 


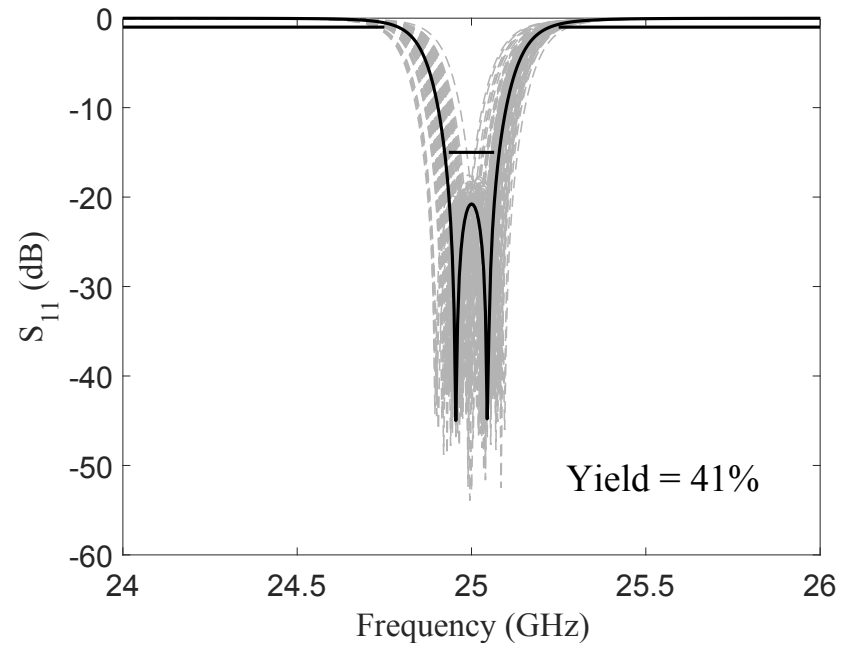

(a)

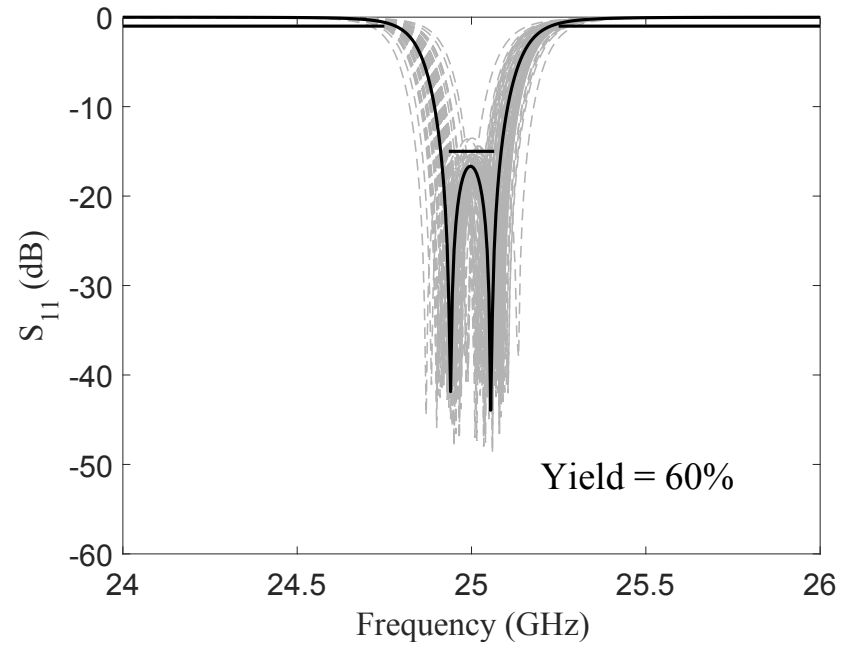

(b)

Figure 5.4: Yield optimization results of the K-band bandpass filter: (a) Before yield optimization and (b) After yield optimization. For both figures, grey dashed lines indicate 100 samples from Monte Carlo analysis, while the black solid line indicates the response evaluated at the nominal point. 


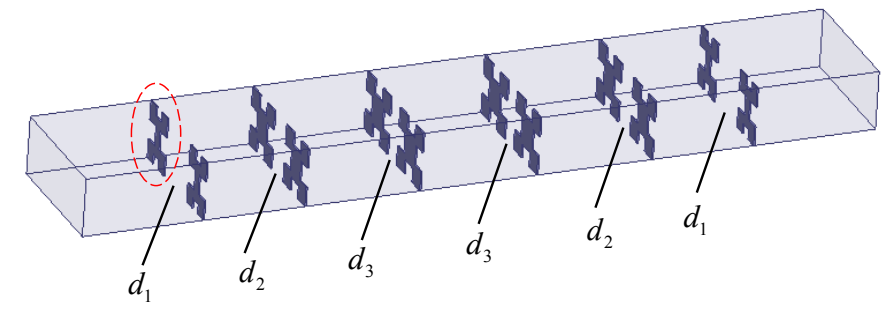

Figure 5.5: The structure of the waveguide bandpass filter example with fractalshaped irises for EM simulation and yield optimization. The three design parameters of the filter are $\boldsymbol{x}=\left[\begin{array}{lll}d_{1} & d_{2} & d_{3}\end{array}\right]^{T}$.

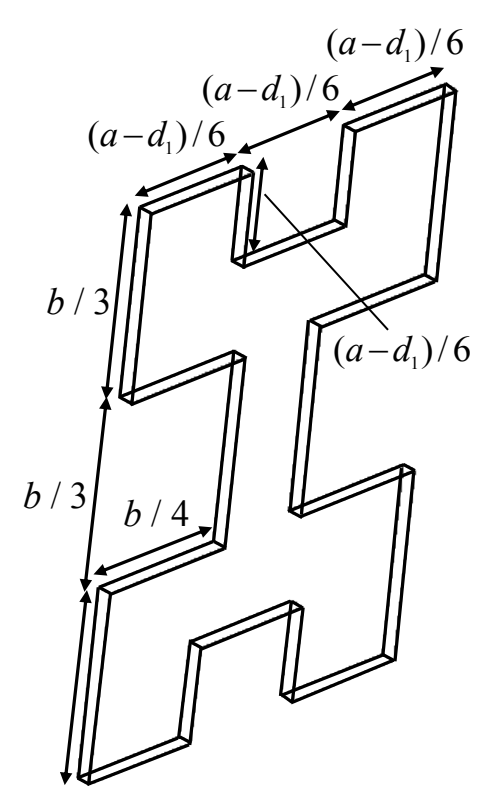

Figure 5.6: Details of the geometrical structure of the fractal shaped iris as circled in Fig. 5.5.

$N=100$ is suitable to represent the statistics of all the possible outcomes. For comparison purpose, different number of random samples $(N=19,50,100)$ per iteration are used for Monte Carlo-based yield optimization approach. The maximum iteration count is set to be a large number to allow both optimization approaches 
Table 5.3: Comparison of Yield Optimization Results for the Waveguide FSI Filter

\begin{tabular}{c|c|c|c|c|c}
\hline \hline $\begin{array}{c}\text { Yield } \\
\text { optimization } \\
\text { approach }\end{array}$ & $\begin{array}{c}\text { Number of EM } \\
\text { simulations } \\
\text { per iteration }\end{array}$ & $\begin{array}{c}\text { Initial } \\
\text { yield }\end{array}$ & $\begin{array}{c}\text { Final } \\
\text { yield }\end{array}$ & $\begin{array}{c}\text { Total number } \\
\text { of EM } \\
\text { simulations }\end{array}$ & $\begin{array}{c}\text { Total } \\
\text { CPU } \\
\text { time }\end{array}$ \\
\hline \multirow{2}{*}{$\begin{array}{c}\text { Monte } \\
\text { Carlo-based }\end{array}$} & 19 & $67 \%$ & $69 \%$ & 304 & $1 \mathrm{~h} 27 \mathrm{~min}$ \\
\cline { 2 - 6 } & 50 & $67 \%$ & $72 \%$ & 900 & $4 \mathrm{~h} 18 \mathrm{~min}$ \\
\cline { 2 - 6 } & 100 & $67 \%$ & $79 \%$ & 1100 & $6 \mathrm{~h} 15 \mathrm{~min}$ \\
\hline Proposed & 19 & $67 \%$ & $84 \%$ & 475 & $2 \mathrm{~h} 16 \mathrm{~min}$ \\
\hline \hline
\end{tabular}

to converge.

Table 5.3 shows a comparison between the two approaches in terms of the final yield, the total number of EM simulations, and the total CPU time required during optimization. From the table we can conclude that the proposed method achieves the greatest yield increase among four cases of optimizations compared. It can also be observed that to achieve a similar yield increase, the total number of EM simulations required by the proposed approach is less than half of that required by the Monte Carlo-based yield optimization approach. This reduction allows us to achieve a substantial speed up for the overall EM-based yield optimization process. Fig. 5.7 shows the yield of the waveguide FSI filter before and after optimization using the proposed approach. The optimal yield solution found by the proposed approach is $\left[\begin{array}{l}9.36824 .95423 .7585\end{array}\right]^{T}(\mathrm{~mm})$.

To further demonstrate the advantages of the proposed approach, we perform another numerical experiment by deliberately stopping the Monte Carlo-based yield optimizations at a certain iteration so that the total number of EM simulations used 


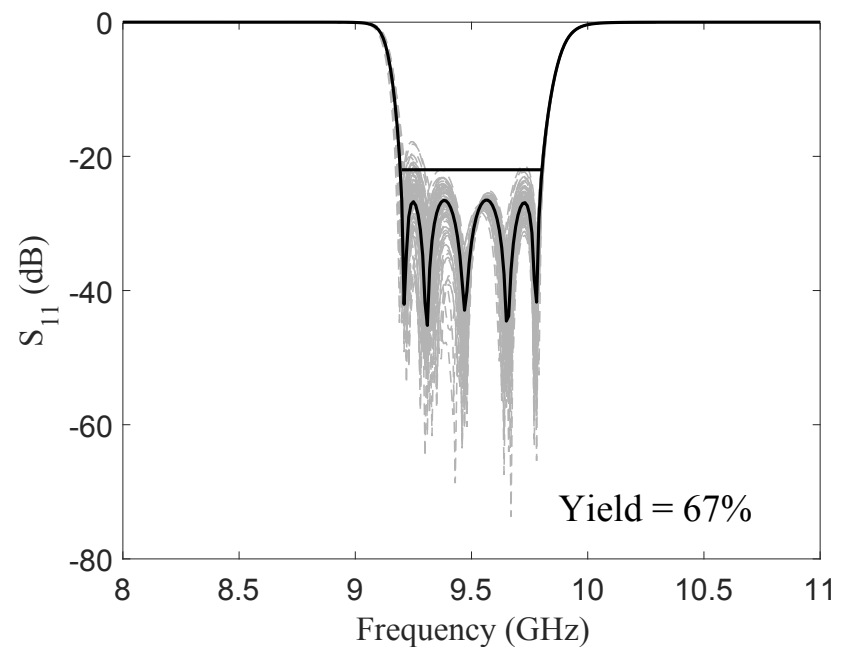

(a)

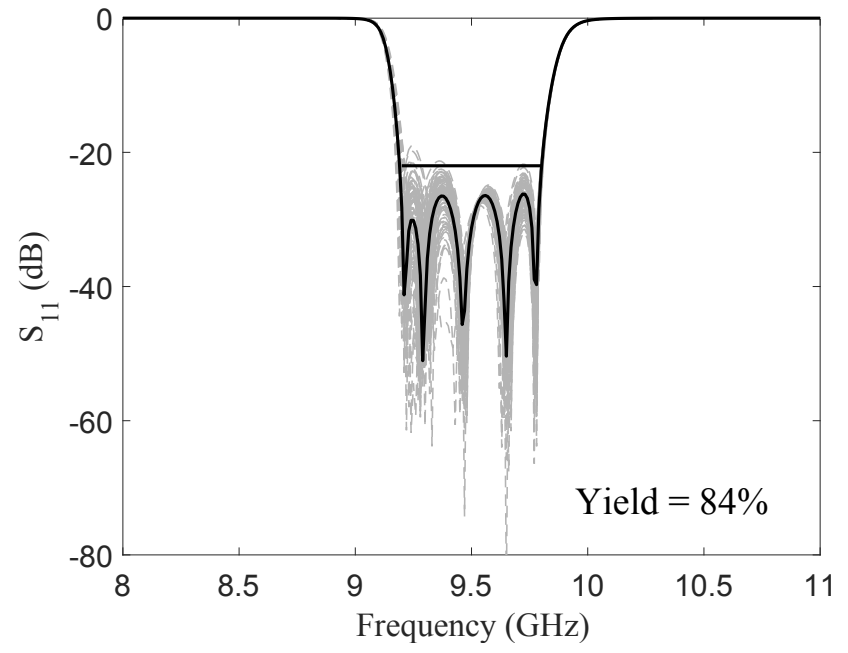

(b)

Figure 5.7: Yield optimization results of the waveguide bandpass filter with fractalshaped irises: (a) Before yield optimization and (b) After yield optimization. Grey dashed lines indicate 100 samples from Monte Carlo analysis, while the black solid line indicates the response evaluated at the nominal point. 
Table 5.4: Comparison of Yield Improvements With Similar Number of EM Simulations for the Waveguide FSI Filter

\begin{tabular}{c|c|c|c|c}
\hline \hline $\begin{array}{c}\text { Yield } \\
\text { optimization } \\
\text { approach }\end{array}$ & $\begin{array}{c}\text { Number of EM } \\
\text { simulations } \\
\text { per iteration }\end{array}$ & Initial yield & Final yield & $\begin{array}{c}\text { Total number } \\
\text { of EM } \\
\text { simulations }\end{array}$ \\
\hline $\begin{array}{c}\text { Monte } \\
\text { Carlo-based }\end{array}$ & 50 & $67 \%$ & $70 \%$ & 450 \\
\cline { 2 - 5 } Proposed & 100 & $67 \%$ & $75 \%$ & 500 \\
\hline \hline
\end{tabular}

is similar to that in the proposed approach. The yield improvements achieved by the two approaches under this condition are shown in Table 5.4. It can be seen from the table that using similar number of EM simulations, the proposed approach achieves a greater yield improvement than the Monte Carlo-based yield optimization approach. The reason is that the proposed PCE-based objective function provides more accurate yield representation and thus more effective direction and step size for updating the nominal point than the conventional Monte Carlo-based yield optimization approach.

\subsubsection{Yield Optimization of a Four-Pole Waveguide Filter}

Lastly, the proposed approach is applied to yield optimization of a four-pole waveguide filter [120]. The structure of the waveguide filter is shown in Fig. 5.8, with five design parameters $\boldsymbol{x}=\left[h_{1} h_{2} h_{3} h_{c 1} h_{c 2}\right]^{T}(\mathrm{~mm}) . h_{1}, h_{2}$, and $h_{3}$ represent the heights of posts in the coupling windows, while $h_{c 1}$ and $h_{c 2}$ are the heights of posts in the resonant cavities. The thickness of all the coupling windows is set to be $2 \mathrm{~mm}$. The design specification is given by $\left|S_{11}\right| \leq-16 \mathrm{~dB}$, in the 


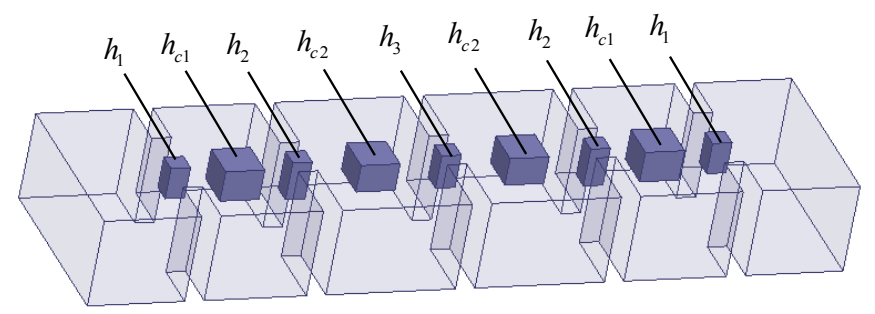

Figure 5.8: The structure of the four-pole waveguide filter example for EM simulation and yield optimization. The five design parameters of the filter are $\boldsymbol{x}=\left[\begin{array}{lllll}h_{1} & h_{2} & h_{3} & h_{c 1} & h_{c 2}\end{array}\right]^{T}$.

frequency range from $10.85 \mathrm{GHz}$ to $11.15 \mathrm{GHz}$. The optimal nominal solution $\boldsymbol{x}_{\text {ini }}^{0}=\left[\begin{array}{ll}3.407 & 4.083 \\ 3 & .5713 .2952 .978\end{array}\right]^{T}(\mathrm{~mm})$ is obtained by performing nominal EM optimization [120].

The design parameters are assumed to be independently normal distributed around their nominal values with 10 microns standard deviation. Before performing yield optimization, Monte Carlo analysis is used to estimate the yield at the optimal nominal solution. By varying the number of random samples from 51 to 300, we observe a convergence on the yield value (53\%) when $N=300$. For this example, Hermite polynomials are used and the accuracy level required is 3 for the sparse grid technique. This results in the number of sparse grid samples $M=51$.

Yield optimization is then performed using the proposed approach with $M=51$. For comparison purpose, we also use the Monte Carlo-based yield optimization approach with $N=51,75,100,200$ to optimize the yield. The final yield is verified by performing a Monte Carlo analysis with 300 random samples at the optimal yield solution. Fig. 5.9 shows the initial yield and the yield after optimization 


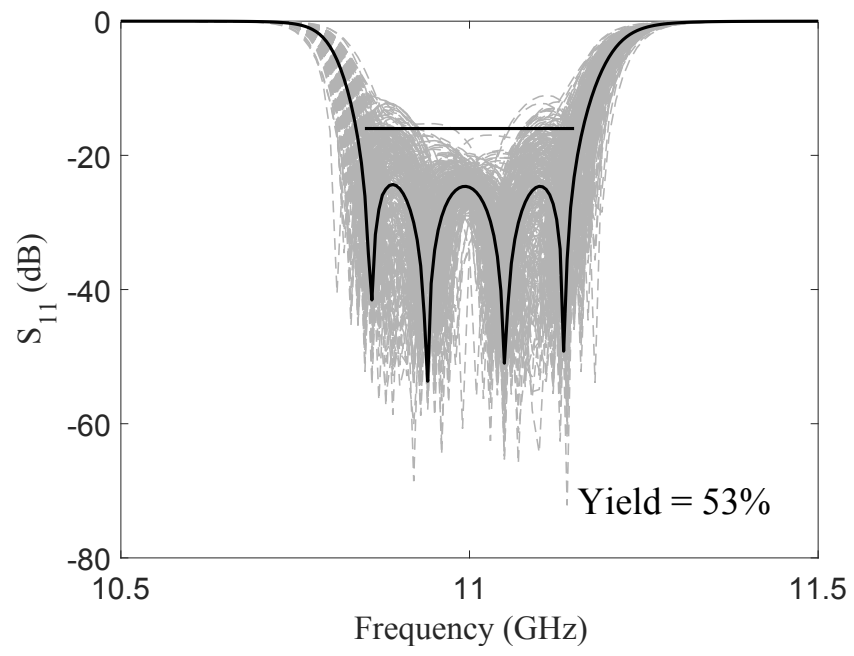

(a)

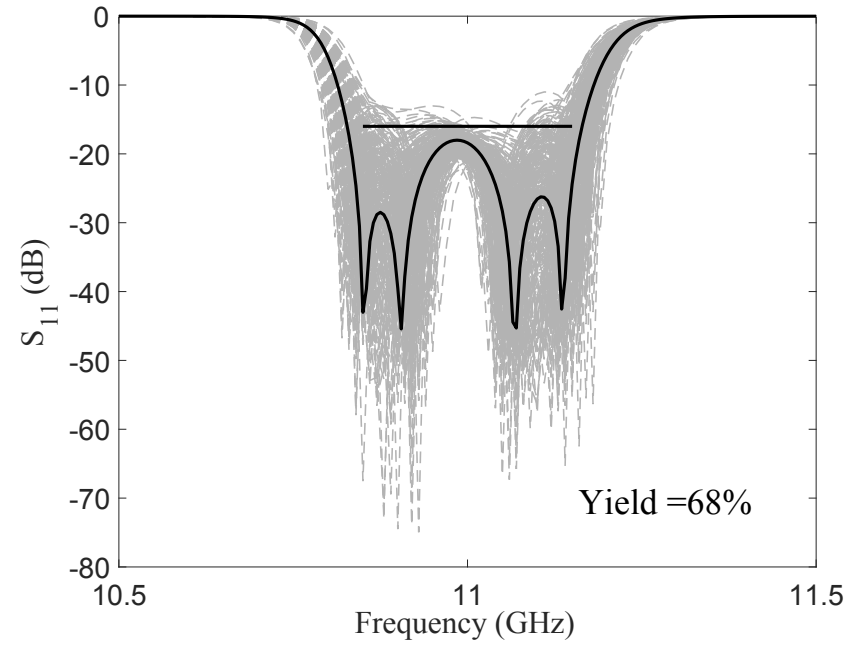

(b)

Figure 5.9: Yield optimization results of the four-pole waveguide filter: (a) Before and (b) After yield optimization. Grey dashed lines indicate 300 samples from Monte Carlo analysis, while the black solid line indicates the response evaluated at the nominal point. 
Table 5.5: Comparison of Yield Optimization Results for the Four-Pole Waveguide Filter

\begin{tabular}{c|c|c|c|c|c}
\hline \hline $\begin{array}{c}\text { Yield } \\
\text { optimization } \\
\text { approach }\end{array}$ & $\begin{array}{c}\text { Number of EM } \\
\text { simulations } \\
\text { per iteration }\end{array}$ & $\begin{array}{c}\text { Initial } \\
\text { yield }\end{array}$ & $\begin{array}{c}\text { Final } \\
\text { yield }\end{array}$ & $\begin{array}{c}\text { Total number } \\
\text { of EM } \\
\text { simulations }\end{array}$ & $\begin{array}{c}\text { Total } \\
\text { CPU } \\
\text { time }\end{array}$ \\
\hline \multirow{3}{*}{$\begin{array}{c}\text { Monte } \\
\text { Carlo-based }\end{array}$} & 51 & $53 \%$ & $58 \%$ & 1173 & $3 \mathrm{~h} 5 \mathrm{~min}$ \\
\cline { 2 - 6 } & 75 & $53 \%$ & $62 \%$ & 1575 & $4 \mathrm{~h} 8 \mathrm{~min}$ \\
\cline { 2 - 6 } & 100 & $53 \%$ & $63 \%$ & 2800 & $7 \mathrm{~h} 21 \mathrm{~min}$ \\
\hline Proposed & 51 & $53 \%$ & $67 \%$ & 4400 & $11 \mathrm{~h} 33 \mathrm{~min}$ \\
\hline \hline
\end{tabular}

using the proposed approach for this example. The optimal yield solution found by the proposed approach is [3.3913 4.14643 .62413 .30192 .9776$]^{T}(\mathrm{~mm})$. The yield optimization results are summarized in Table 5.5 and Table 5.6. It can be observed from Table 5.5 that the proposed approach achieves the greatest yield increase among the five cases of optimizations compared. To achieve a similar yield increase, the proposed approach requires much fewer EM simulations and thus much shorter CPU time than the Monte Carlo-based yield optimization approach. A substantial speed up for the overall yield optimization process has been achieved. As a further comparison shown in Table 5.6, it can be observed that using similar number of EM simulations, the proposed approach achieves a greater yield improvement than the Monte Carlo-based yield optimization approach. 
Table 5.6: Comparison of Yield Improvements With Similar Number of EM Simulations for the Four-Pole Waveguide Filter

\begin{tabular}{c|c|c|c|c}
\hline \hline $\begin{array}{c}\text { Yield } \\
\text { optimization } \\
\text { approach }\end{array}$ & $\begin{array}{c}\text { Number of EM } \\
\text { simulations } \\
\text { per iteration }\end{array}$ & Initial yield & Final yield & $\begin{array}{c}\text { Total number } \\
\text { of EM } \\
\text { simulations }\end{array}$ \\
\hline $\begin{array}{c}\text { Monte } \\
\text { Carlo-based }\end{array}$ & 75 & $53 \%$ & $58 \%$ & 1275 \\
\cline { 2 - 5 } & 100 & $53 \%$ & $60 \%$ & 1300 \\
\hline Proposed & 51 & $53 \%$ & $64 \%$ & 1400 \\
\hline \hline
\end{tabular}

\subsection{Conclusions and Summary}

In this work, a novel polynomial chaos-based approach to yield-driven EM optimization has been proposed. The computational advantages of the polynomial chaos approach have been exploited to facilitate EM-based yield optimization of microwave structures. The PCE coefficients have been incorporated into the formulation of the optimization objective function such that the number of EM simulations required to obtain effective update direction and suitable step size of the nominal point is reduced. Sensitivity formulas have been derived for both the PCE coefficients and the proposed objective function w.r.t. the yield optimization variables. Compared with the conventional Monte Carlo-based yield optimization approach, the proposed approach is able to achieve similar yield increase using much fewer EM simulations or greater yield increase with similar number of EM simulations. The proposed approach helps to achieve high-quality solutions in shorter time for the challenging problem of yield-driven EM optimization. 


\section{Chapter 6}

\section{Conclusions and Future Research}

\subsection{Conclusions}

In this thesis, a novel training approach for parametric modeling of microwave passive components with respect to changes in geometrical parameters has been developed. A novel parallel space mapping based yield-driven EM optimization approach incorporating trust region algorithm and PCE has been presented. To address the situation when no equivalent circuit coarse models are available, another PCE-based approach has been further proposed for yield-driven EM optimization.

This thesis has first proposed a novel training approach for developing parametric models of microwave passive components using Padé via Lanczos and EM sensitivities. The EM responses (e.g., $S$-parameters) of passive components versus frequency have been represented by pole-zero-gain transfer functions. To address the issue of mismatch of poles/zeros, a novel sensitivity-analysis-based pole/zeromatching algorithm has been proposed. The proposed algorithm utilizes EM sensitivities, which provides useful information for the direction of movement of the 
poles/zeros, to predict the new positions of the poles/zeros for each change of geometrical parameters. The predicted new positions have been used to guide the matching process of poles/zeros between different geometrical parameter values. In comparison with the existing methods, the proposed algorithm obtains more reliable pole/zero-matching solutions when geometrical variations are large. This allows the trained neural networks to have more accurate predictions for the poles and zeros subject to large geometrical variations, and ultimately increases the accuracy and robustness of the overall model. The proposed approach obtains better accuracy in challenging applications involving large geometrical variations.

A novel parallel space-mapping based yield-driven EM optimization technique incorporating trust region algorithm and PCE has been proposed to facilitate yielddriven EM optimization. A trust region algorithm has been proposed to update the trust radius of each design parameter in each space mapping iteration during optimization. Parallel computation method has been used to generate the EM data in parallel and enable surrogate modeling over multiple geometrical samples. The trained surrogate has been used to find the new yield optimal solution using the $l_{1}$ centering algorithm. The PCE approach has been incorporated to further speed up the yield verification on fine models.

To address the situation when equivalent circuit coarse models are not available, a novel polynomial chaos expansion based approach has been further developed. The PCE coefficients have been incorporated into the formulation of the optimization objective function such that the number of EM simulations required to obtain effective update direction and suitable step size of the nominal point is reduced. 
Sensitivity formulas have been derived for both the PCE coefficients and the proposed objective function w.r.t. the yield optimization variables. Compared with the conventional Monte Carlo-based yield optimization approach, the proposed approach is able to achieve similar yield increase using much fewer EM simulations or greater yield increase with similar number of EM simulations.

\subsection{Future Research}

Some possible future research directions based on the extension of the approaches proposed in this thesis are identified as follows

- One of the future research directions is to apply the proposed parametric modeling approach to accelerate EM-based yield estimation and EM-based yield optimization. With the proposed approach, we can train a surrogate model to accurately represent the EM behavior of a given microwave component in a certain region in the design parameter space. The region can be either a relatively small region around the initial nominal point to allow reliable yield estimations or a large region to enable yield-driven design. Once an accurate parametric model is developed, Monte Carlo analysis can be performed on the surrogate model to realize yield estimation, and gradient-based optimization algorithms can be employed to find the optimal yield solution iteratively.

- Another future research direction is to extend the use of the proposed training approach from pure EM parametric modeling to multi-physics parametric modeling of microwave passive components. High performance microwave 
design often calls for the consideration of the behavior of microwave components in a multi-physics environment. Under this consideration, multi-physics simulations, which provide comprehensive bi-directional analyses between different physics domains (such as EM, thermal, and structural mechanics etc.), are typically required. However, the computational costs of multi-physics simulations are usually very high as it involves the analyses of the behavior of the microwave component in multiple domains. This problem becomes more severe when considering multiphysics optimizations, since repetitive changes of design parameter values necessitates massive number of multiphysics simulations. Using the proposed training approach, parametric models that are able to provide fast and accurate prediction of the multiphysics behavior of microwave components can be developed. The developed parametric models can then be utilized for high-level circuit and system designs in a multiphysics environment.

- The surrogate-assisted yield optimization approach has been demonstrated by microstrip filter examples in this thesis. Yield-driven design of other microwave passive components such as diplexers and antennas also involves high computational costs. Another future direction is to apply the proposed parallel SM based yield optimization approach to EM-based yield-driven design of other types of microwave passive components, such as antennas. To do this, suitable equivalent circuit coarse models need to be found. In case that an equivalent circuit coarse model is not easy to be established, coarse- and finemesh SM can be utilized where coarse-mesh simulation is used as the coarse 
model and fine-mesh simulation is used as the fine model.

- As another further research, we would like to expand the use of the proposed PCE-based yield optimization approach for applications involving high dimensional design parameter spaces. One of the difficulties for such an expansion is that the computational complexity of PCE coefficients increases dramatically as the number of statistical parameters increases. To reduce the number of sparse grid samples required in computing the PCE coefficients, one possible solution is to apply the principal component analysis (PCA) method to convert the original possibly correlated variables into a set of linearly uncorrelated variables. Another solution is to use the sensitivities of the stochastic output (i.e., EM response) with respect to the statistical variables in the computation of PCE coefficients. The adjoint sensitivity approach can also be employed in the computation of sensitivity of the stochastic output. 


\section{Appendix}

In this appendix, we provide the detailed derivations of the transfer function in the complex propagation space, as given by Equation (3.1). This transfer function is to be used in the proposed training approach for parametric modeling of microwave passive components in Chapter 3.

\section{Formulation of the FEM}

Let $\mu_{r}$ and $\epsilon_{r}$ represent relative permeability and relative permittivity, respectively. Application of the FEM to time-harmonic field problems deals with the following vector wave equation [96]:

$$
\nabla \times\left(\frac{1}{\mu_{r}} \nabla \times \mathbf{E}\right)-k_{0}^{2} \epsilon_{r} \mathbf{E}=-j k_{0} Z_{0} \boldsymbol{J}
$$

where $\mathbf{E}$ represents the electric field vector and $k_{0}=\sqrt{\omega^{2} \mu_{0} \epsilon_{0}} . \quad \mu_{0}$ and $\epsilon_{0}$ are permeability and permittivity of the free space, respectively. $Z_{0}$ is the impedance of free space and $\boldsymbol{J}$ represents current density.

In the following descriptions, we take the problem of waveguide discontinuities as an example to show our derivations. Let $S_{1}$ and $S_{2}$ denote the input port and output port for the waveguide, respectively. The boundary conditions for the problem 
include:

$$
\begin{aligned}
& \hat{n} \times(\nabla \times \mathbf{E})+\gamma \hat{n} \times(\hat{n} \times \mathbf{E})=\mathbf{U}^{i n c} \text {, on } S_{1} \\
& \hat{n} \times(\nabla \times \mathbf{E})+\gamma \hat{n} \times(\hat{n} \times \mathbf{E})=0, \quad \text { on } S_{2} \\
& \hat{n} \times \mathbf{E}=0, \quad \text { on waveguide wall }
\end{aligned}
$$

where $\gamma=j \beta$ and $\beta$ is the propagation constant. For example, for rectangular waveguides, $\beta=\sqrt{\omega^{2} \mu_{0} \epsilon_{0}-(\pi / a)^{2}}$, where $a$ is the width of the waveguide. $\mathbf{U}^{i n c}=$ $-2 j \beta \mathbf{E}^{i n c}$, where $\mathbf{E}^{i n c}$ represents an incident wave. By following the generalized variational principle, we obtain [96]

$$
\begin{aligned}
\boldsymbol{K}^{e} & =\iiint_{V^{e}}\left[\frac{1}{\mu_{r}^{e}}\left\{\nabla \times \mathbf{N}^{e}\right\} \cdot\left\{\nabla \times \mathbf{N}^{e}\right\}^{T}\right. \\
& \left.\quad-k_{0}^{2} \epsilon_{r}^{e}\left\{\mathbf{N}^{e}\right\} \cdot\left\{\mathbf{N}^{e}\right\}^{T}\right] \mathrm{d} V, \\
\boldsymbol{B}^{s} & =\iint_{s^{s}} \gamma\left\{\mathbf{S}^{s}\right\} \cdot\left\{\mathbf{S}^{s}\right\}^{T} \mathrm{~d} S, \\
\boldsymbol{b}^{s} & =\iint_{s^{s}}\left\{\hat{n} \times \mathbf{S}^{s}\right\} \cdot \mathbf{U}^{i n c} \mathrm{~d} S,
\end{aligned}
$$

and the following linear system [96]:

$$
\boldsymbol{K} e=\boldsymbol{b},
$$

where $\mathbf{N}^{e}$ represents the $3-\mathrm{D}$ vector basis functions, and $\mathbf{S}^{s}$ represents the $2-\mathrm{D}$ vector basis functions. The system matrix $\boldsymbol{K}$ is assembled from $\boldsymbol{K}^{e}$ and $\boldsymbol{B}^{s}$, while the system right hand side $\boldsymbol{b}$ is assembled from $\boldsymbol{b}^{s}$. Both $\boldsymbol{K}$ and $\boldsymbol{b}$ change as geometrical parameter values of the EM structure change. $\boldsymbol{e}$ is the vector containing all the degree of freedoms (DoFs) used in approximating the electric field $\mathbf{E}$ in the 
solution domain.

Once we have solved (6.4) for the electric fields on $S_{1}$ and $S_{2}$, the $S$-parameters $S_{11}$ and $S_{21}$ can be calculated as follows [96]:

$$
\begin{aligned}
& S_{11}=\frac{2 e^{-j \beta z_{1}}}{a b E_{0}} \iint_{S_{1}} \mathbf{E}\left(x, y, z_{1}\right) \cdot \boldsymbol{y}_{10}(x, y) \mathrm{d} S-e^{-2 j \beta z_{1}}, \\
& S_{21}=\frac{2 e^{j \beta z_{2}}}{a b E_{0}} \iint_{S_{2}} \mathbf{E}\left(x, y, z_{2}\right) \cdot \boldsymbol{y}_{10}(x, y) \mathrm{d} S
\end{aligned}
$$

where $a$ and $b$ denote the width and height of the waveguide, respectively. $z_{1}$ and $z_{2}$ are the $z$-coordinates of the input and output ports, respectively. $E_{0}$ denotes the magnitude of the incident wave, and $\boldsymbol{y}_{10}$ is given by

$$
\boldsymbol{y}_{10}=\hat{y} \sin \frac{\pi x}{a}
$$

where $\hat{y}$ represents the unit vector of the $y$-axis.

\section{Application of MPVL to the FEM System}

As a frequency-domain model-order reduction technique, the MPVL algorithm allows fast frequency sweeps by obtaining a transfer matrix $\boldsymbol{H}(s)$ through solving the FEM system at a single frequency point. MPVL solves problems where the 
equations describing the system can be expressed as follows [97]:

$$
\begin{aligned}
\boldsymbol{H}(s) & =\boldsymbol{L}^{T}(\boldsymbol{G}+s \boldsymbol{C})^{-1} \boldsymbol{B}, \\
s & =s_{0}+\sigma, \\
\boldsymbol{A} & =-\left(\boldsymbol{G}+s_{0} \boldsymbol{C}\right)^{-1} \boldsymbol{C}, \\
\boldsymbol{R} & =\left(\boldsymbol{G}+s_{0} \boldsymbol{C}\right)^{-1} \boldsymbol{B}, \\
\boldsymbol{H}\left(s_{0}+\sigma\right) & =\boldsymbol{L}^{T}(\boldsymbol{I}-\sigma \boldsymbol{A})^{-1} \boldsymbol{R},
\end{aligned}
$$

where $s$ is the Laplace frequency variable, $s_{0}$ is the point of expansion in the complex $s$ plane, and $\boldsymbol{L}$ is a collection of vectors that choose the outputs of interest from the unknowns $\boldsymbol{e}$. The matrices $\boldsymbol{G}, \boldsymbol{C}$, and $\boldsymbol{B}$ are all in general complex-valued matrices whose elements are independent of $s$.

The linear system in (6.4) can be cast into the following form [121]:

$$
\left(\boldsymbol{K}_{0}+\gamma \boldsymbol{K}_{1}+\gamma^{2} \boldsymbol{K}_{2}\right) \boldsymbol{e}=\gamma \boldsymbol{b}_{1}
$$

where $\boldsymbol{K}_{0}, \boldsymbol{K}_{1}$, and $\boldsymbol{K}_{2}$ are all sparse matrices independent of the frequency but dependent on the values of geometrical parameters. $\boldsymbol{b}_{1}$ is a column matrix describing the specific excitation and is independent of the frequency. To apply the MPVL algorithm to solve (6.8) for fast frequency sweep, the following definitions are made 
[97]:

$$
\begin{aligned}
\boldsymbol{G} & =\left[\begin{array}{cc}
\boldsymbol{0}_{M \times M} & \boldsymbol{I}_{M \times M} \\
\boldsymbol{K}_{0} & \boldsymbol{K}_{1}
\end{array}\right], \\
\boldsymbol{C} & =\left[\begin{array}{cc}
-\boldsymbol{I}_{M \times M} & \mathbf{0}_{M \times M} \\
\mathbf{0}_{M \times M} & \boldsymbol{K}_{2}
\end{array}\right], \\
\hat{\boldsymbol{b}}_{1} & =\left[\begin{array}{c}
\boldsymbol{0}_{M \times 1} \\
\boldsymbol{b}_{1}
\end{array}\right], \\
\boldsymbol{A} & =-\left(\boldsymbol{G}+\gamma_{0} \boldsymbol{C}\right)^{-1} \boldsymbol{C}, \\
\boldsymbol{r} & =\left(\boldsymbol{G}+\gamma_{0} \boldsymbol{C}\right)^{-1} \hat{\boldsymbol{b}}_{1}, \\
\gamma & =\gamma_{0}+\sigma,
\end{aligned}
$$

where $M$ is the total number of degree of freedoms (DoFs) for the original FEM system; $\sigma=\gamma-\gamma_{0}$, and $\gamma_{0}$ is the point of expansion in the complex $\gamma$ plane.

Given $\boldsymbol{G}, \boldsymbol{C}, \hat{\boldsymbol{b}}_{1}, \boldsymbol{A}$, and $\boldsymbol{r}$ defined above, the results returned from the MPVL algorithm are the $q \times q$ tridiagonal matrix $\boldsymbol{T}$, the $q \times 1$ vector $\boldsymbol{\rho}$, and the $q \times N_{\text {in }}$ matrix $\boldsymbol{\eta}$, where $q$ is the order of the ROMs. For the calculation of $S$-parameters (e.g., $S_{11}$ ), we are interested in the electric fields on the waveguide ports. Let the unknowns (DoFs) on the input port $\left(S_{1}\right)$ be denoted by $\boldsymbol{E}_{S_{1}}(\gamma) \in \mathbb{C}^{N_{i n} \times 1}$, where $N_{i n}$ is the number of unknowns on the input port. Then, by using the MPVL algorithm, $\boldsymbol{E}_{S_{1}}$ is obtained as follows [97]:

$$
\boldsymbol{E}_{S_{1}}\left(\gamma_{0}+\sigma\right)=\boldsymbol{\eta}^{T}(\boldsymbol{I}-\sigma \boldsymbol{T})^{-1} \boldsymbol{\rho},
$$


Note that since $\boldsymbol{G}, \boldsymbol{C}$, and $\hat{\boldsymbol{b}}_{1}$ all depend on geometrical parameter values, the elements of $\boldsymbol{T}, \boldsymbol{\rho}$, and $\boldsymbol{\eta}$ will change as geometrical parameter values change. Once $\boldsymbol{E}_{S_{1}}$ has been found by using the MPVL algorithm, the $S$-parameters (or, the transfer functions) can be calculated. For example, the $S_{11}$ can be obtained as follows (assume $z_{1}=0.0$ and $E_{0}=1.0$ )

$$
S_{11}=\frac{2}{a b} \times \boldsymbol{\alpha}^{T} \gamma \boldsymbol{E}_{S_{1}}-1.0
$$

where $\boldsymbol{\alpha}^{T}$ represents a constant vector whose elements depend on the coordinates of the nodes in the FEM mesh.

\section{Transformation of the Transfer Function}

Next, we perform eigendecomposition to obtain two matrices $\boldsymbol{Q} \in \mathbb{C}^{q \times q}$ and $\boldsymbol{\Lambda} \in$ $\mathbb{C}^{q \times q}$ as follows [23]:

$$
\boldsymbol{T}=\boldsymbol{Q} \boldsymbol{\Lambda} \boldsymbol{Q}^{-1}=\boldsymbol{Q} \operatorname{diag}\left(\lambda_{1}, \lambda_{1}, \ldots, \lambda_{q}\right) \boldsymbol{Q}^{-1}
$$

Substituting (6.12) into (6.10) yields

$$
\boldsymbol{E}_{S_{1}}=\boldsymbol{\eta}^{T} \boldsymbol{Q}(\boldsymbol{I}-\sigma \boldsymbol{\Lambda})^{-1} \boldsymbol{Q}^{-1} \boldsymbol{\rho}
$$

Let $\hat{\boldsymbol{\alpha}}=\boldsymbol{\alpha}^{T} \boldsymbol{\eta}^{T} \boldsymbol{Q}$ and $\boldsymbol{\beta}=\boldsymbol{Q}^{-1} \boldsymbol{\rho}$, where $\hat{\boldsymbol{\alpha}} \in \mathbb{C}^{1 \times q}$ and $\boldsymbol{\beta} \in \mathbb{C}^{q \times 1}$. Let $\hat{\alpha}_{i}$ and $\beta_{i}$ be the $i$ th $(i=1, \ldots, q)$ element in $\hat{\boldsymbol{\alpha}}$ and the $i$ th element in $\boldsymbol{\beta}$, respectively. 
Substituting (6.13) into (6.11), we obtain

$$
\begin{aligned}
S_{11} & =\frac{2}{a b} \gamma \hat{\boldsymbol{\alpha}}(\boldsymbol{I}-\sigma \boldsymbol{\Lambda})^{-1} \boldsymbol{\beta}-1.0 \\
& =\frac{2}{a b} \gamma \sum_{i=1}^{q} \frac{\mu_{i}}{1-\sigma \lambda_{i}}-1.0 \\
& =\frac{2}{a b} \gamma \sum_{i=1}^{q} \frac{r_{i}}{\sigma-p_{i}}-1.0,
\end{aligned}
$$

where $\mu_{i}=\hat{\alpha}_{i} \beta_{i}, r_{i}=-\mu_{i} / \lambda_{i}$, and $p_{i}=1 / \lambda_{i}, i=1, \ldots, q$. The symbols $p_{i}$ and $r_{i}$ represent the poles and residues in the transfer function in the " $\gamma$-space", respectively. The values of the poles and residues change as geometrical parameters change. By transforming the pole-residue form into the pole-zero-gain form, we obtain $z_{i}$ and $K[122]$. The $S_{11}$ expressed in pole-zero-gain form is

$$
S_{11}(\omega)=\gamma(\omega) K \frac{\prod_{i=1}^{q-1}\left(\sigma(\omega)-z_{i}\right)}{\prod_{i=1}^{q}\left(\sigma(\omega)-p_{i}\right)}-1.0,
$$

where $\sigma(\omega)$ is defined as $\sigma(\omega)=\gamma(\omega)-\gamma_{0}$. Equation (6.15) coincides with the transfer function given by (3.1). Other $S$-parameters can be calculated in a similar manner. 


\section{References}

[1] Q. J. Zhang, K. C. Gupta, and V. K. Devabhaktuni, "Artificial neural networks for RF and microwave design - From theory to practice," IEEE Trans. Microw. Theory Techn., vol. 51, no. 4, pp. 1339-1350, Apr. 2003.

[2] V. Rizzoli, A. Costanzo, D. Masotti, A. Lipparini, and F. Mastri, "Computeraided optimization of nonlinear microwave circuits with the aid of electromagnetic simulation," IEEE Trans. Microw. Theory Techn., vol. 52, no. 1, pp. 362377, Jan. 2004.

[3] J. E. Rayas-Sánchez, "EM-based optimization of microwave circuits using artificial neural networks: The state-of-the-art," IEEE Trans. Microw. Theory Techn., vol. 52, no. 1, pp. 420-435, Jan. 2004.

[4] M. B. Steer, J. W. Bandler, and C. M. Snowden, "Computer-aided design of RF and microwave circuits and systems," IEEE Trans. Microw. Theory Techn., vol. 50, no. 3, pp. 996-1005, Mar. 2002.

[5] F. Mkadem and S. Boumaiza, "Physically inspired neural network model for RF power amplifier behavioral modeling and digital predistortion," IEEE Trans. Microw. Theory Techn., vol. 59, no. 4, pp. 913-923, Apr. 2011. 
[6] D. E. Root, "Future device modeling trends," IEEE Microw. Mag., vol. 13, no. 7, pp. 45-59, Nov./Dec. 2012.

[7] H. Yu, H. Chalamalasetty, and M. Swaminathan, "Modeling of voltagecontrolled oscillators including I/O behavior using augmented neural networks," IEEE Access, vol. 7, pp. 38973-38982, Mar. 2019.

[8] A. D. Huang, Z. Zhong, W. Wu, and Y. X. Guo, "An artificial neural networkbased electrothermal model for GaN HEMTs with dynamic trapping effects consideration," IEEE Trans. Microw. Theory Techn., vol. 64, no. 8, pp. 2519-2528, Aug. 2016.

[9] S. Liao, H. Kabir, Y. Cao, J. Xu, Q. J. Zhang, and J. Ma, "Neural-network modeling for 3-D substructures based on spatial EM-field coupling in finiteelement method," IEEE Trans. Microw. Theory Techn., vol. 59, no. 1, pp. 21-38, Jan. 2011.

[10] S. A. Sadrossadat, Y. Cao, and Q. J. Zhang, "Parametric modeling of microwave passive components using sensitivity-analysis-based adjoint neuralnetwork technique," IEEE Trans. Microw. Theory Techn., vol. 61, no. 5, pp. 1733-1747, May 2013.

[11] Y. Cao and G. Wang, "A wideband and scalable model of spiral inductors using space-mapping neural network," IEEE Trans. Microw. Theory Techn., vol. 55, no. 12 , pp. 2473-2480, Dec. 2007. 
[12] J. E. Rayas-Sánchez and V. Gutierrez-Ayala, "EM-based Monte Carlo analysis and yield prediction of microwave circuits using linear-input neural-output space mapping," IEEE Trans. Microw. Theory Techn., vol. 54, no. 12, pp. 4528-4537, Dec. 2006.

[13] W. Na and Q. J. Zhang, "Automated knowledge-based neural network modeling for microwave applications," IEEE Microw. Wireless Compon. Lett., vol. 24, no. 7, pp. 499-501, Jun. 2014.

[14] J. W. Bandler, M. A. Ismail, J. E. Rayas-Sánchez, and Q. J. Zhang, "Neuromodeling of microwave circuits exploiting space-mapping technology," IEEE Trans. Microw. Theory Techn., vol. 47, no. 12, pp. 2417-2427, Dec. 1999.

[15] V. K. Devabhaktuni, B. Chattaraj, M. C. E. Yagoub, and Q. J. Zhang, "Advanced microwave modeling framework exploiting automatic model generation, knowledge neural networks, and space mapping," IEEE Trans. Microw. Theory Techn., vol. 51, no. 7, pp. 1822-1833, Jul. 2003.

[16] H. Kabir, L. Zhang, M. Yu, P. H. Aaen, J. Wood, and Q. J. Zhang, "Smart modeling of microwave device," IEEE Microw. Mag., vol. 11, no. 3, pp. 105-118, May 2010.

[17] Y. Cao, G. Wang, and Q. J. Zhang, "A new training approach for parametric modeling of microwave passive components using combined neural networks and transfer functions," IEEE Trans. Microw. Theory Techn., vol. 57, no. 11, pp. 2727-2742, Nov. 2009. 
[18] V. M. R. Gongal-Reddy, F. Feng, and Q. J. Zhang, "Parametric modeling of millimeter-wave passive components using combined neural networks and transfer functions," in Proc. Global Symposium on Millimeter Waves (GSMM), Montreal, QC, Canada, May 2015, pp. 1-3.

[19] Z. Guo, J. Gao, Y. Cao, and Q. J. Zhang, "Passivity enforcement for passive component modeling subject to variations of geometrical parameters using neural networks," in Proc. IEEE MTT-S Int. Microw. Symp. Dig., Montreal, QC, Canada, Jun. 2012, pp. 1-3.

[20] F. Feng, C. Zhang, J. Ma, and Q. J. Zhang, "Parametric modeling of EM behavior of microwave components using combined neural networks and poleresidue-based transfer functions," IEEE Trans. Microw. Theory Techn., vol. 64, no. 1, pp. 60-77, Jan. 2016.

[21] F. Feng, V. M. R. Gongal-Reddy, C. Zhang, J. Ma, and Q. J. Zhang, "Parametric modeling of microwave components using adjoint neural networks and pole-residue transfer functions with EM sensitivity analysis," IEEE Trans. Microw. Theory Techn., vol. 65, no. 6, pp. 1955-1975, Jun. 2017.

[22] B. Gustavsen and A. Semlyen, "Rational approximation of frequency domain responses by vector fitting," IEEE Trans. Power Del., vol. 14, no. 3, pp. 10521061, Jul. 1999.

[23] Y. Yue, L. Feng, and P. Benner, "Interpolation of reduced-order models based on modal analysis," IEEE Int. Conf. on Numerical Electromagnetic and Mul- 
tiphysics Modeling and Optimization (NEMO), Reykjavik, Iceland, Aug. 2018, pp. 1-4.

[24] Y. Yue, L. Feng, and P. Benner, "An adaptive method for interpolating reduced-order models based on matching and continuation of poles," IEEE Int. Conf. on Numerical Electromagnetic and Multiphysics Modeling and Optimization (NEMO), Cambridge, MA, May 2019, pp. 1-4.

[25] J. W. Bandler and S. H. Chen, "Circuit optimization: The state of the art," IEEE Trans. Microw. Theory Techn., vol. 36, no. 2, pp. 424-443, Feb. 1988.

[26] R. Biernacki, S. Chen, G. Estep, J. Rousset, and J. Sifri, "Statistical analysis and yield optimization in practical RF and microwave designs," IEEE MTT-S Int. Microwave Symp. Dig., Montreal, QC, 2012, pp. 1-3.

[27] J. W. Bandler, J. E. Rayas-Sanchez, and Q. J. Zhang, "Yield-driven electromagnetic optimization via space mapping-based neuromodels," Int. J. RF Microw. Comput.-Aided Eng., vol. 12, no. 1, pp. 79-89, Jan. 2002.

[28] H. L. Abdel-Malek, A. S. O. Hassan, E. A. Soliman, and S. A. Dakroury, "The ellipsoidal technique for design centering of microwave circuits exploiting spacemapping interpolating surrogates," IEEE Trans. Microw. Theory Techn., vol. 54, no. 10, pp. 3731-3738, Oct. 2006.

[29] Q. S. Cheng, J. W. Bandler, and S. Koziel, "Response corrected tuning space mapping for yield estimation and design centering," IEEE MTT-S Int. Microw. Symp. Dig., Anaheim, CA, 2010, pp. 193-196. 
[30] A. S. Hassan, A. S. Mohamed, and A. Y. El-Sharabasy, "Statistical microwave circuit optimization via a non-derivative trust region approach and space mapping surrogates," IEEE MTT-S Int. Microwave Symp. Dig., Baltimore, MD, 2011, pp. 1-4.

[31] S. Koziel and A. Bekasiewicz, "Low-cost surrogate-assisted statistical analysis of miniaturized microstrip couplers," J. Electromagnetic Waves Appl., vol. 30, no. 10, pp. 1345-1353, 2016.

[32] C. Zhang, W. Na, Q. J. Zhang, and J. W. Bandler, "Fast yield estimation and optimization of microwave filters using a cognition-driven formulation of space mapping," IEEE MTT-S Int. Microw. Symp. Dig., San Francisco, CA, 2016, pp. $1-4$.

[33] J. Zhang, K. Ma, F. Feng, and Q. J. Zhang, "Distributed parallel computing technique for EM modeling." IEEE Int. Conf. on Numerical Electromagnetic and Multiphysics Modeling and Optimization for RF, Microwave, and Terahertz Applications (NEMO), Ottawa, Canada, Aug. 2015.

[34] J. Zhang, K. Ma, F. Feng, and Q. J. Zhang, "Parallel gradient-based local search accelerating particle swarm optimization for training microwave neural network models," IEEE MTT-S Int. Microw. Symp. Dig., Phoenix, AZ, May 2015, pp. 1-3. 
[35] F. Feng, C. Zhang, V.-M.-R. Gongal-Reddy, Q. J. Zhang, and J. Ma, "Parallel space-mapping approach to EM optimization," IEEE Trans. Microw. Theory Techn., vol. 62, no. 5, pp. 1135-1148, May 2014.

[36] D. Xiu and G. Karniadakis, "The Wiener-Askey polynomial chaos for stochastic differential equations," SIAM J. Sic. Comput., vol. 24, no. 2, pp. 619-644, Feb. 2002.

[37] A. C. M. Austin, and C. D. Sarris, "Efficient analysis of geometrical uncertainty in the FDTD method using polynomial chaos with application to microwave circuits," IEEE Trans. Microw. Theory Techn., vol. 61, no. 12, pp. 4293-4301, Dec. 2013.

[38] T.-A. Pham, E. Gad, M. S. Nakhla, and R. Achar, "Decoupled polynomial chaos and its applications to statistical analysis of high-speed interconnects," IEEE Trans. Compon. Packag., Manuf. Technol., vol. 4, no. 10, pp. 1634-1647, Oct. 2014.

[39] A. C. M. Austin, N. Sood, J. Siu, and C. D. Sarris, "Application of polynomial chaos to quantify uncertainty in deterministic channel models," IEEE Trans. Antennas Propag., vol. 61, no. 11, pp. 5754-5761, Nov. 2013.

[40] P. Sumant, H. Wu, A. Cangellaris, and N. Aluru, "Reduced-order models of finite element approximations of electromagnetic devices exhibiting statistical variability," IEEE Trans. Antennas Propag., vol. 60, no. 1, pp. 301-309, Jan. 2012. 
[41] D. V. Ginste, D. De Zutter, D. Deschrijver, T. Dhaene, P. Manfredi, and F. Canavero, "Stochastic modeling-based variability analysis of on-chip interconnects," IEEE Trans. Compon. Packag., Manuf. Technol., vol. 2, no. 7, pp. 1182-1192, Jul. 2012.

[42] P. Manfredi, D. V. Ginste, D. De Zutter, and F. G. Canavero, "Improved polynomial chaos discretization schemes to integrate interconnects into design environments," IEEE Microw. Wireless Compon. Lett., vol. 23, no. 3, pp. 116118, Mar. 2013.

[43] A. K. Prasad, M. Ahadi, and S. Roy, "Multidimensional uncertainty quantification of microwave/RF networks using linear regression and optimal design of experiments," IEEE Trans. Microw. Theory Techn., vol. 64, no. 8, pp. 2433-2446, Aug. 2016.

[44] M. Ahadi and S. Roy, "Sparse linear regression (SPLINER) approach for efficient multidimensional uncertainty quantification of high-speed circuits," IEEE Trans. Comput.-Aided Design Integr. Circuits Syst., vol. 35, no. 10, pp. 16401652, Oct. 2016.

[45] P. Manfredi, D. V. Ginste, D. De Zutter, and F. G. Canavero, "Uncertainty assessment of lossy and dispersive lines in SPICE-type environments," IEEE Trans. Compon. Packag., Manuf. Technol., vol. 3, no. 7, pp. 1252-1258, Jul. 2013. 
[46] M. R. Rufuie, E. Gad, M. Nakhla, and R. Achar, "Generalized Hermite polynomial chaos for variability analysis of macromodels embedded in nonlinear circuits," IEEE Trans. Compon. Packag., Manuf. Technol., vol. 4, no. 4, pp. 673-684, Apr. 2014.

[47] A. K. Prasad and S. Roy, "Accurate reduced dimensional polynomial chaos for efficient uncertainty quantification of microwave/RF networks," IEEE Trans. Microw. Theory Techn., vol. 65, no. 10, pp. 3697-3708, Oct. 2017.

[48] J. Zhang, F. Feng, W. Zhang, J. Jin, J. Ma, and Q. J. Zhang, "A novel training approach for parametric modeling of microwave passive components using Padé via Lanczos and EM sensitivities," IEEE Trans. Microw. Theory Techn., submitted, Aug. 2019.

[49] J. Zhang, F. Feng, W. Na, S. Yan, and Q. J. Zhang, "Parallel space mapping based yield-driven EM optimization incorporating trust region algorithm and polynomial chaos expansion," IEEE Access, vol. 7, no. 1, pp. 143673-143683, Sep. 2019.

[50] J. Zhang, C. Zhang, F. Feng, W. Zhang, J. Ma, and Q. J. Zhang, "Polynomial Chaos-Based Approach to Yield-Driven EM Optimization," IEEE Trans. Microw. Theory Techn., vol. 66, no. 7, pp. 3186-3199, May 2018.

[51] W. C. Na and Q. J. Zhang, "Automated parametric modeling of microwave components using combined neural network and interpolation techniques," in IEEE MTT-S Int. Dig., Seattle, WA, USA, Jun. 2013. 
[52] G. Gosal, E. Almajali, D. McNamara and M. Yagoub, "Transmitarray antenna design using forward and inverse neural network modeling," IEEE Ant. and Wireless Prop. Lett., vol. 15, no. 1, pp. 1483-1486, Dec. 2015.

[53] P. M. Watson and K. C. Gupta, "EM-ANN models for microstrip vias and interconnects in multilayer circuits," IEEE Trans. Microw. Theory Techn., vol. 44, no. 12, pp. 2495-2503, Dec. 1996.

[54] Q. J. Zhang and M. S. Nakhla, "Signal integrity analysis and optimization of VLSI interconnects using neural network models," IEEE Int. Circuits Syst. Symp., London, England, May 1994, pp. 459-462.

[55] A. Veluswami, M. S. Nakhla, and Q. J. Zhang, "The application of neural networks to EM-based simulation and optimization of interconnects in highspeed VLSI circuits," IEEE Trans. Microw. Theory Techn., vol. 45, no. 5, pp. 712-723, May 1997.

[56] F. Wang and Q. J. Zhang, "Knowledge based neural models for microwave design," IEEE Trans. Microw. Theory Techn., vol. 45, no. 12, pp. 2333-2343, Dec. 1997.

[57] V. K. Devabhaktuni, C. Xi, F. Wang, and Q. J. Zhang, "Robust training of microwave neural models," Int. J. RF Microwave CAE, vol. 12, no. 1, pp. 109124, Jan. 2002. 
[58] P. M. Watson and K. C. Gupta, "Design and optimization of CPW circuits using EM-ANN models for CPW components," IEEE Trans. Microw. Theory Techn., vol. 45, no. 12, pp. 2515-2523, Dec. 1997.

[59] G. Fedi, S. Manetti, G. Pelosi, and S. Selleri, "Design of cylindrical posts in rectangular waveguide by neural network approach," IEEE AP-S Int. Symp. Digest, Salt Lake City, UT, Jul. 2000, pp. 1054-1057.

[60] X. Ding, V. K. Devabhaktuni, B. Chattaraj, M. C. E. Yagoub, M. Doe, J. J. Xu, and Q. J. Zhang, "Neural-network approaches to electromagnetic-based modeling of passive components and their applications to high-frequency and high-speed nonlinear circuit optimization," IEEE Trans. Microw. Theory Tech., vol. 52, no. 1, pp. 436-449, Jan. 2004.

[61] V. K. Devabhaktruni, M. Yagoub, and Q. J. Zhang, "A robust algorithm for automatic development of neural-network models for microwave applications," IEEE Trans. Microw. Theory Techn., vol. 49, no. 12, pp. 2282-2291, Dec. 2001.

[62] Y. Fang, M. C. E. Yagoub, F. Wang, and Q. J. Zhang, "A new macromodeling approach for nonlinear microwave circuits based on recurrent neural networks," IEEE Trans. Microw. Theory Techn., vol. 48, no. 12, pp. 2335-2344, Dec. 2000.

[63] J. W. Bandler, M. A. Ismail, J. E. Rayas-Sanchez, and Q. J. Zhang, "Neuromodeling of microwave circuits exploiting space-mapping technology," IEEE Trans. Microw. Theory Techn., vol. 47, no. 12, pp. 2417-2427, Dec. 1999. 
[64] G. L. Creech, B. J. Paul, C. D. Lesniak, T. J. Jenkins, and M. C. Calcatera, "Artificial neural networks for fast and accurate EM-CAD of microwave circuits," IEEE Trans. Microwave Theory Techn., vol. 45, no. 5, pp. 794-802, May 1997.

[65] W. Na, W. Zhang, S. Yan, and G. Liu, "Automated neural-based modeling of microwave devices using parallel computation and interpolation approaches," IEEE Access, vol. 7, pp. 73929-73937, May 2019.

[66] S. Yan, Y. Zhang, X. Jin, W. Zhang, and W. Shi, "Multi-physics parametric modeling of microwave passive components using artificial neural networks," Progress In Electromagnetics Research M, vol. 72, pp. 79-88, Aug. 2018.

[67] W. Zhang, F. Feng, V. M. R. Gongal-Reddy, J. Zhang, S. Yan, J. Ma, and Q. J. Zhang, "Space mapping approach to electromagnetic centric multiphysics parametric modeling of microwave components," IEEE Trans. Microw. Theory Techn., vol. 66, no. 7, pp. 3169-3185, Jul. 2018.

[68] V. Rizzoli, A. Neri, D. Masotti, and A. Lipparini, "A new family of neural network-based bidirectional and dispersive behavioral models for nonlinear RF/microwave subsystems," Int. J. RF Microw. Computer-Aided Eng., vol. 12, no. 1, pp. 51-70, Jan. 2002.

[69] Y. Cao, J. J. Xu, V. K. Devabhaktuni, R. T. Ding, and Q. J. Zhang, "An adjoint dynamic neural network technique for exact sensitivities in nonlinear 
transient modeling and high-speed interconnect design," IEEE MTT-S Int. Microw. Symp. Dig., PA, Philadelphia, Jun. 2003, pp. 165-168.

[70] J. Xu, M. C. E. Yagoub, R. Ding, and Q. J. Zhang, "Neural based dynamic modeling of nonlinear microwave circuits," IEEE Trans. Microw. Theory Techn., vol. 50, no. 12, pp. 2769-2780, Dec. 2002.

[71] I. S. Stievano, I. A. Maio, and F. G. Canavero, "Parametric macromodels of digital I/O ports," IEEE Trans. Adv. Packag., vol. 25, no. 5, pp. 255-264, May 2002.

[72] M. Isaksson, D. Wisell, and D. Ronnow, "Wide-band dynamic modeling of power amplifiers using radial-basis function neural networks," IEEE Trans. Microw. Theory Techn., vol. 53, no. 11, pp. 3422-3428, Nov. 2005.

[73] B. O'Brien, J. Dooley, and T. J. Brazil, "RF power amplifier behavioral modeling using a globally recurrent neural network," IEEE MTT-S Int. Microw. Symp. Dig., San Francisco, CA, Jun. 2006, pp. 1089-1092.

[74] Y. H. Fang, M. C. E. Yagoub, F.Wang and Q. J. Zhang, "A new macromodeling approach for nonlinear microwave circuits based on recurrent neural networks," IEEE Trans. Microw. Theory Tech., vol. 48, no. 12, pp. 2335-2344, Dec. 2000.

[75] S. Yan, C. Zhang, and Q. J. Zhang, "Recurrrent neural network technique for behavioral modeling of power amplifier with memory effects," Int. J. RF Microwave CAE, vol. 25, no. 4, pp. 289-298, Oct. 2014. 
[76] T. Liu, S. Boumaiza, and F. M. Ghannouchi, "Dynamic behavioral modeling of 3G power amplifiers using real-valued time-delay neural networks," IEEE Trans. Microw. Theory Techn., vol. 52, no. 3, pp. 1025-1033, Mar. 2004.

[77] Y. Cao, R. T. Ding, and Q. J. Zhang, "A new nonlinear transient modeling technique for high-speed integrated circuit applications based on state-space dynamic neural network," IEEE MTT-S Int. Microw. Symp. Dig., Fort Worth, TX, Jun. 2004, pp. 1553-1556.

[78] Y. Cao, R. T. Ding, and Q. J. Zhang, "State-space dynamic neural network technique for high-speed IC applications: Modeling and stability analysis," IEEE Trans. Microw. Theory Techn., vol. 54, no. 6, pp. 2398-2409, Jun. 2006.

[79] J. Jin, C. Zhang, F. Feng, W. Na, J. Ma, and Q. J. Zhang, "Deep neural network technique for high-dimensional microwave modeling and applications to parameter extraction of microwave filters," IEEE Trans. Microw. Theory Techn., DOI: 10.1109/TMTT.2019.2932738.

[80] D. E. Hocevar, M. R. Lightner, and T. N. Trick, "An extrapolated yield approximation for use in yield maximization," IEEE Trans. Comput.-Aided Design Integr. Circuits Syst., vol. CAD-3, no. 4, pp. 279-287, Oct. 1984.

[81] A. H. Zaabab, Q. J. Zhang, and M. Nakhla, "A neural network modeling approach to circuit optimization and statistical design," IEEE Trans. Microw. Theory Techn., vol. 43, no. 6, pp. 1349-1358, Jun. 1995. 
[82] K. Singhal and J. F. Pinel, "Statistical design centering and tolerancing using parametric sampling," IEEE Trans. Circuits Syst., vol. CAS-28, no. 7, pp. 692702, Jul. 1981.

[83] H. L. Abdel-Malek, A. S. O. Hassan, and M. H. Bakr, "Statistical circuit design with the use of a modified ellipsoidal technique," Int. J. Microw. Millim.-Wave Comput.-Aided Eng., vol. 7, no. 1, pp. 117-129, Jan. 1997.

[84] H. L. Abdel-Malek and J. W. Bandler, "Yield estimation for efficient design centering assuming arbitrary statistical distributions," Int. J. Circuit Theory Applicat., vol. 6, no. 3, pp. 289-303, Jul., 1978.

[85] H. L. Abdel-Malek, A. S. O. Hassan, and M. H. Bakr, "A boundary gradient search technique and its application in design centering," IEEE Trans. Comput.Aided Design Integr. Circuits Syst., vol. 18, no. 11, pp. 1654-1661, Nov. 1999.

[86] J. W. Bandler, R. M. Biernacki, S. H. Chen, P. A. Grobelny, and R. H. Hemmers, "Space mapping technique for electromagnetic optimization," IEEE Trans. Microwave Theory Tech., vol. 42, no. 12, pp. 2536-2544, Dec. 1994.

[87] M. A. Ismail, D. Smith, A. Panariello, Y. Wang, and M. Yu, "EM-based design of large-scale dielectric-resonator filters and multiplexers by space mapping," IEEE Trans. Microw. Theory Techn., vol. 52, no. 1, pp 386-392, Jan. 2004.

[88] J. V. Morro et al., "Fast automated design of waveguide filters using aggressive space mapping with a new segmentation strategy and a hybrid optimization 
algorithm," IEEE Trans. Microw. Theory Techn., vol. 53, no. 4, pp. 1130-1142, Apr. 2005.

[89] N. K. Nikolova, X. Zhu, Y. Song, A. Hasib, and M. H. Bakr, "S-parameter sensitivities for electromagnetic optimization based on volume field solutions," IEEE Trans. Microw. Theory Techn., vol. 57, no. 6, pp. 1526-1538, Jun. 2009.

[90] M. L. Stein, "Large sample properties of simulations using Latin Hypercube sampling," Technometrics, vol. 29, pp. 143-151, May 1987.

[91] J. W. Bandler, R. M. Biernacki, S. H. Chen, P. A. Grobelny, and R. H. Hemmers, "Exploitation of coarse grid for electromagnetic optimization," IEEE MTT-S Int. Microwave Symp. Dig., San Diego, CA, 1994, pp. 381-384.

[92] S. Koziel and J. W. Bandler, "Rapid yield estimation and optimization of microwave structures exploiting feature-based statistical analysis," IEEE Trans. Microw. Theory Techn., vol. 63, no. 1, pp. 107-114, Jan. 2015.

[93] M. S. Eldred, "Recent advances in non-intrusive polynomial chaos and stochastic collocation methods for uncertainty analysis and design," in Proceedings of Structures, Structural Dynamics, and Materials Conference, Palm Springs, CA, May 2009, pp. 1-20.

[94] F. Heiss and V. Winschel, "Likelihood approximation by numerical integration on sparse grids," J. Econometr., vol. 144, no. 1, pp. 62-80, May 2008.

[95] J. Zhang, C. Zhang, F. Feng, W. Zhang, and Q. J. Zhang, "Recent advances in EM-based yield estimation," IEEE Int. Conf. on Numerical Electromagnetic 
and Multiphysics Modeling and Optimization for RF, Microwave, and Terahertz Applications (NEMO), Reykjavik, Iceland, Aug. 2018.

[96] J. M. Jin, The Finite Element Method in Electromagnetics. New York, NY, USA: Wiley, 2002.

[97] R. D. Slone and R. Lee, "Applying Padé via Lanczos to the finite element method for electromagnetic radiation problems," Radio Sci., vol. 35, no. 2, pp. 331-340, Mar.-Apr. 2000.

[98] S. Koziel and A. Bekasiewicz, "Fast EM-driven optimization using variablefidelity EM models and adjoint sensitivities," IEEE Microw. Wireless Compon. Lett., vol. 26, no. 2, pp. 80-82, Feb. 2016.

[99] C. Geuzaine and J. F. Remacle, "Gmsh: a three-dimensional finite element mesh generator with built-in pre- and post-processing facilities," International Journal for Numerical Methods in Engineering 79(11), pp. 1309-1331, 2009.

[100] F. Feng, J. Zhang, W. Zhang, Z. Zhao, J. Jin, and Q. J. Zhang, "Coase and fine mesh space mapping for EM optimization incorporating mesh deformation," IEEE Microw. Wireless Compon. Lett., vol. 29, no. 8, pp. 510-512, Aug. 2019.

[101] A. Lamecki, "A mesh deformation technique based on solid mechanics for parametric analysis of high-frequency devices with 3-D FEM," IEEE Trans. Microw. Theory Techn., vol. 64, no. 11, pp. 3400-3408, Nov. 2016.

[102] D. C. Montgomery, Design and Analysis of Experiments. New York: Wiley, 1991. 
[103] J. W. Bandler, Q. S. Cheng, S. A. Dakroury, A. S. Mohamed, M. H. Bakr, K.Madsen, and J. Søndergaard, "Space mapping: The state of the art," IEEE Trans. Microw. Theory Techn., vol. 52, no. 1, pp. 337-361, Jan. 2004.

[104] S. Koziel, S. Ogurtsov, J. W. Bandler, and Q. S. Cheng, "Reliable spacemapping optimization integrated with EM-based adjoint sensitivities," IEEE Trans. Microw. Theory Techn., vol. 61, no. 10, pp. 3493-3502, Oct. 2013.

[105] S. Koziel, Q. S. Cheng, and J. W. Bandler, "Fast EM modeling exploiting shape-preserving response prediction and space mapping," IEEE Trans. Microw. Theory Techn., vol. 62, no. 3, pp. 399-407, Mar. 2014.

[106] J. W. Bandler, J. E. Rayas-Sanchez, and Q. J. Zhang, "Yield-driven electromagnetic optimization via space mapping-based neuromodels," Int. J. RF Microwave CAE, vol. 12, no. I, pp. 79-89, Jan. 2002.

[107] Q. S. Cheng, J. W. Bandler, and S. Koziel. "Response corrected tuning space mapping for yield estimation and design centering." IEEE MTT-S Int. Microwave Symp. Dig., Anaheim, CA, 2010, pp. 193-196.

[108] A. S. Hassan, A. S. Mohamed, and A. Y. El-Sharabasy. "Statistical microwave circuit optimization via a non-derivative trust region approach and space mapping surrogates." IEEE MTT-S Int. Microwave Symp. Dig., Baltimore, MD, 2011, pp. 1-4. 
[109] V. Gongal-Reddy, S. Zhang, C. Zhang, and Q. J. Zhang, "Parallel computational approach to gradient based EM optimization of passive microwave circuits," IEEE Trans. Microw. Theory Techn., vol. 64, no. 1, pp. 44-59, Jan. 2016.

[110] Z. Zhang, T. A. El-Moselhy, I. M. Elfadel, and L. Daniel, "Stochastic testing method for transistor-level uncertainty quantification based on generalized polynomial chaos," IEEE Trans. Comput.-Aided Design Integr. Circuits Syst., vol. 32, no. 10, pp. 1533-1545, Oct. 2013.

[111] X. Du, L. Leifsson, and S. Koziel, "Rapid multi-band patch antenna yield estimation using polynomial chaos-Kriging," International Conference on Computational Science, Faro, Portugal, Jun. 2019, pp. 487-494.

[112] A. H. Zaabab, Q. J. Zhang, and M. S. Nakhla, "A neural network modeling approach to circuit optimization and statistical design," IEEE Trans. Microwave Theory Tech., vol. 43, pp. 1349-1358, Jun. 1995.

[113] X. Li, J. Le, P. Gopalakrishnan, and L. T. Pileggi, "Asymptotic probability extraction for non-normal distributions of circuit performance," in Proc. Int. Conf. Computer-Aided Design, Nov. 2004, pp. 2-9.

[114] Z. Zhang, N. Farnoosh, T. Klemas, and L. Daniel, "Maximum-entropy density estimation for MRI stochasitc surrogate models," IEEE Trans. Antennas Propag., vol. 13, pp. 1656-1659, Aug. 2014. 
[115] N. M. Steen, G. D. Byrne, and E. M. Gelbard, "Gauss quadratures for the integrals $\int_{0}^{\infty} \exp \left(-x^{2}\right) f(x) d x$ and $\int_{0}^{b} \exp \left(-x^{2}\right) f(x) d x, "$ Math. Comput., vol. 23, pp. 661-671, 1969 .

[116] A. Papoulis, Probability, Random Variables and Stochastic Processes. New York: McGraw-Hill, 1991.

[117] D. Xiu, "Efficient collocational approach for parametric uncertainty analysis," Commun. Comput. Phys., vol. 2, no. 2, pp. 293-309, 2007.

[118] J. Zhang, K. Ma, F. Feng, and Q. J. Zhang, "Distributed parallel computing technique for EM modeling," IEEE Int. Conf. on Numerical Electromagnetic Modeling and Optimization for RF, Microwave, and Terahertz Applications (NEMO), Ottawa, ON, Aug. 2015.

[119] D. Oloumi, A. Kordzadeh, and A. A. Lotfi Neyestanak, "Size reduction and bandwidth enhancement of a waveguide bandpass filter using fractal-shaped irises," IEEE Antennas Wirel. Propag. Lett., vol. 8, pp. 1214-1217, Oct. 2009.

[120] C. Zhang, F. Feng, V. Gongal-Reddy, Q. J. Zhang, and J. W. Bandler, "Cognition-driven formulation of space mapping for equal-ripple optimization of microwave filters," IEEE Trans. Microw. Theory Techn., vol. 63, no. 7, pp. 2154-2165, Jul. 2015.

[121] J. Gong and J. L. Volakis, "AWE implementation for electromagnetic FEM analysis," Electron. Lett., vol. 32, pp. 2216-2217, Nov. 1996. 
[122] A. J. Laub and B. C. Moore. "Calculation of transmission zeros using QZ techniques," Automatica, vol. 14, no. 6, pp. 557-566, Nov. 1978. 